\title{
Determining the viability of the use of magneto-rheological fluid in a low cost stroke rehabilitation device
}

by

\author{
Abigail Rajendran
}

\author{
A thesis \\ submitted to the Victoria University of Wellington \\ in fulfilment of the \\ requirements for the degree of \\ Master of Science \\ in Electronics and Computer Systems Engineering. \\ Victoria University of Wellington \\ 2013
}





\begin{abstract}
There are over 15 million people affected by strokes worldwide with a third left disabled. It is estimated that only 5 to $20 \%$ regain upper limb functionality. However, research has shown that repetitive movement on the affected limb improves motor relearning. With the number of people affected by strokes rising each year the demand has begun straining hospital resources, therefore there is a need for some therapy to be moved away from clinical settings and into a person's home. Robot assisted therapy is a growing field aiming to meet this demand. However currently there are no low cost devices able to actively exercise and strengthen a person's hand during the acute (early) stage of stroke rehabilitation.

This study is a part of a larger project involving the development of a low cost, assistive stroke rehabilitation device requiring a controllable damper. The aim of the study is to determine whether the use of magnetorheological fluid in a controllable damper is viable for use in the planned rehabilitation device. A rotary damper configuration was chosen as it can be made compact and avoid fluid leakage. To be deemed suitable for the application, the viscous torque of the damper needed to be controllable with varying input current. The required damping torques produced must be repeatable and needs to be generated below $34^{\circ} \mathrm{C}$, the specified maximum operating temperature of the system.

The performance of three vane designs for the rotary damper were investigated. These three designs were layered discs, a paddle and a helix. A test rig using a pulley configuration was designed and constructed to quantify the performance of the vane designs. The test rig recorded the opposing force and temperature measurements for each damper design. The
\end{abstract}


measurements of interest were the off-state (no input current) torque, the achievable torque range, and also the consistency of the measurements.

Experiments were conducted with the damper containing air to determine the pre-existing friction between the vane and housing, and water and motor oil were used as the damper fluid to investigate the performance of the designs with known fluid viscosities. Lastly experiments containing magneto-rheological fluid were conducted to determine the controllability and consistency of the viscous torque of each design.

The paddle design was selected based on its range and consistency of produced torque, simplicity of the design and expected economical manufacture. With an input current of 0 to $2 \mathrm{~A}$ the damper produced a viscous torque range of $0.0036 \mathrm{Nm}$ to $0.044 \mathrm{Nm}$, which was the equivalent opposing force of approximately $7.3 \mathrm{~N}$.

During testing of the various damper designs, a few imperfections were found. A modified version of the chosen damper was constructed to determine whether those features were manufacturing artifacts. It was found that the force measurements became smoother and previous periodic oscillations in the measurements were eliminated.

The viscous torque of the paddle design was found to be controllable within the given operational conditions and therefore the use of magnetorheological fluid is a viable solution for use in a low cost stroke rehabilitation device. 


\section{Acknowledgments}

First and foremost, praises and thanks be to God, for the wisdom, guidance and many blessings throughout my life. Through whom even the unimaginable becomes a possibility.

I would like to express my deep and sincere gratitude to my research supervisors Dr Will Browne and Dr Christopher Hollitt, for the opportunity to have continued on from my previous research. Their support, enthusiasm and dedication throughout the research has been incredible and our insightful yet humourous meetings will be sorely missed!

Thank you to Mr Sunil Vather and the team at Im-Able Ltd for their industrial expertise in the field of stroke rehabilitation work.

To Ms Sheila Mann and Mr Leslie Austin for sharing their experiences with stroke rehabilitation.

A special thank you to Mr Tim Exley, Mr Sean Anderson and Mr Jason Edwards for all their electronics support.

A thank you to Mr Manu Pouajen-Blakiston and Mr Nick Grinter for their mechanical support and construction of parts.

Thank you to TechNZ for funding the research. 
To family and friends for their prayers, words of encouragement and understanding when at times research work needed to take priority.

A special mention to Dayna Kivell my thesis writing buddy. We both went through this crazy thesis writing time together and made it out the other end!

Finally I am extremely grateful to be blessed which such wonderful parents, whose continuous love, support and sacrifices extend far beyond words. 


\section{Contents}

1 Introduction 1

1.1 Motivation . . . . . . . . . . . . . . . . . . 1

1.2 Scope ....................... 4

1.3 Aim ...................... 5

1.4 Research Objectives . . . . . . . . . . . . . . . 6

1.5 Benefits . . . . . . . . . . . . . . . . 10

2 Background 11

2.1 Existing Stroke Rehabilitation Devices . . . . . . . . . . . . . 11

2.2 Magneto-rheological Fluid . . . . . . . . . . . . . . . . 15

2.2.1 Advantages of MR fluid . . . . . . . . . . . . 16

2.2.2 Challenges of MR fluid . . . . . . . . . . . . 17

2.2.3 Operational modes of MR fluid . . . . . . . . . . . 18

2.3 Applications of MR Fluid . . . . . . . . . . . . . . . . 22

2.3.1 Linear damper . . . . . . . . . . . . . . 22

2.3.2 Rotary damper . . . . . . . . . . . . . 24

2.4 Summary . . . . . . . . . . . . . . . . 26

3 Method 27

3.1 Selection of Damper Configuration . . . . . . . . . . . . . 27

3.1.1 Force requirements . . . . . . . . . . . . . 28

3.1.2 System operating temperature . . . . . . . . . . 28

3.1.3 Size, mass and power consumption . . . . . . . . . 29 
3.1.4 Selected configuration . . . . . . . . . . 29

3.2 Test Rig Designs to Measure Force . . . . . . . . . . . . . 30

3.2 .1 Linear test rig . . . . . . . . . . . . . 30

3.2 .2 Pulley test rig . . . . . . . . . . . . 32

3.3 Component Selection and Test Rig Construction . . . . . . . 34

3.3.1 Force and temperature component selection . . . . . 34

3.3.2 Cord selection . . . . . . . . . . . . . . 36

3.3.3 MR damper pulley and shaft . . . . . . . . 36

3.3 .4 Test rig pulleys . . . . . . . . . . . . . . 37

3.3.5 Motor considerations . . . . . . . . . . . . . . 39

3.3.6 Test rig construction . . . . . . . . . . . . 41

3.4 Damper Housing and Vane Designs . . . . . . . . . . . . 43

3.4.1 Rotary damper housing . . . . . . . . . . 43

3.4.2 Rotary vane designs . . . . . . . . . . . . . 45

3.5 Test Rig Operation . . . . . . . . . . . . . . . . . . . 49

3.5.1 Selection of motor speeds . . . . . . . . . 52

3.6 Data Analysis Procedure . . . . . . . . . . . . . . . . . . 54

3.6.1 Characteristics of force measurements . . . . . . . . 54

3.6.2 Sample size . . . . . . . . . . . . . . 57

3.6.3 Averaging force graphs . . . . . . . . . . . 61

3.6.4 Conversion from force measurements to torque . . . 62

3.6.5 Torque plots statistics . . . . . . . . . . 66

3.7 Summary . . . . . . . . . . . . . . . 70

$\begin{array}{lll}4 & \text { Results } & 71\end{array}$

4.1 Damper with Three Fluids . . . . . . . . . . . . . . 72

4.1.1 Air filled damper results . . . . . . . . . . . 72

4.1.2 Water filled damper results . . . . . . . . . 77

4.1 .3 Oil filled damper results . . . . . . . . . . . . . 77

4.2 Temperature Measurements . . . . . . . . . . . . . . . . 87 
4.2.1 Determining a safe operating temperature range of PLA . . . . . . . . . . . . . . 87

4.2.2 Temperature time response of the system . . . . . . 88

4.3 Damper with MR Fluid . . . . . . . . . . . . . . . . . 92

4.3.1 Force results for the discs design in MR fluid . . . . . 94

4.3.2 Force results for the paddle design in MR fluid . . . . 96

4.3.3 Force results for the helix design in MR fluid . . . . . 99

4.3.4 Calculated torque of the three vane designs with changes in input current . . . . . . . . . . . . . . . . . . . . 101

4.3.5 Selection of the vane design to further develop . . . . 105

4.4 Further Experimentation with Paddle Design . . . . . . . . . 106

4.4.1 Settling of homemade and commercial MR fluid . . . 107

4.4.2 Paddle vane in commercial MR fluid . . . . . . . . . . 107

4.4.3 Modifications to the housing . . . . . . . . . . 110

4.4.4 Results of the two alternative vane designs in magnetorheological fluid . . . . . . . . . . . . . . . . 114

4.4.5 Heating effects on the generated torque of the damper117

4.5 Summary . . . . . . . . . . . . . . . . . . . . . . . . 119

5 Conclusion 123

5.1 Conclusion . . . . . . . . . . . . . . . . 123

5.2 Contribution . . . . . . . . . . . . . . . . . . . 124

5.3 Future Work . . . . . . . . . . . . . . . . . . . . 125

5.4 Final Statement . . . . . . . . . . . . . . . . . . 126

$\begin{array}{ll}\text { References } & 127\end{array}$

$\begin{array}{ll}\text { Appendix } & 133\end{array}$

A LabVIEW Block Diagram 
B Water Filled Damper Results 136

B.1 Water: Discs Design . . . . . . . . . . . . . . . . . 137

B.2 Water: Flat Paddle Design . . . . . . . . . . . 138

B.3 Water: Helix Design . . . . . . . . . . . . . . . . . 139 


\section{Chapter 1}

\section{Introduction}

\subsection{Motivation}

According to the World Health Organization 15 million people worldwide suffer a stroke each year and of those, a third are left disabled [1]. As a result of a severe stroke the neural circuits between the brain and limbs can be weakened or lost.

Research has shown that repetitive movement of an affected limb improves motor relearning [2]. Motor relearning uses the process of neuroplasticity, which is the ability for the brain to learn and adapt, to restructure and strengthen affected neural circuits [3]. However repetitive movement alone is not sufficient. As the brain adapts, the rehabilitation techniques must also adapt, so that the brain remains stimulated and challenged [4].

To develop a device for acute (early stage) stroke, a device able to actively assist is required, due to the limited movements of the patient. Variations in rehabilitation techniques can be introduced by changing the goal orientated task, often through virtual games, and by applying controlled opposing forces, allowing the level of difficulty of the exercise to be altered. Traditional stroke rehabilitation is focused around activities of daily living. Following a severe stroke, muscle control is limited. Ba- 
sic exercises such as opening and closing the hand, are repeated multiple times, to relearn the muscle movement. As movements are relearned, exercises promoting the strengthening of the weakened muscles are introduced. Progress is assessed and exercises are recommended in one-on-one sessions with rehabilitation therapists [5].

The greatest increase in recovery occurs within the first 3-6 months following the stroke. The total recovery process is time consuming, spanning over many months and even years, and session times are often limited and not as frequent as required. Approximately $80 \%$ of stroke survivors are able to learn to walk again, but it is estimated that only 5 to $20 \%$ regain complete upper limb functionality [6]. This may be due to a lack of motivation and frustration leading to overcompensation of the unaffected arm and hand hindering progress.

As the number of people affected by stroke rise each year, the growing demand for rehabilitation has begun straining hospital resources. Stroke survivors are often discharged once they regain enough mobility to walk, even though their affected hand still requires further therapy [7]. Therefore, current rehabilitation processes are in need of more effective tools, allowing neurotherapies to be moved away from the therapy gymnasium and into a person's home [8].

Robot assisted therapy is a rapidly growing field aiming to meet the current demands of rehabilitation. Potential advantages over traditional therapy include increased control of movements, motivational benefits through visual and tactile stimuli, and the ability to track progress both during an exercise session and over time [9].

Several robotic therapies for hand rehabilitation are becoming commercially available. Current passive devices suited for home therapy include the Able-X [10] and HandTutor [11]. These devices are targeted towards the later stages of rehabilitation where some motor control in the affected hand has already been regained. The Amadeo [12] is a commercial device able to actively assist finger movements. However the large and 
costly device, is designed for use in hospital and clinical environments, under supervision of therapists. Assistive devices still under development include the HWARD [13] and the Gentle/G system [14]. For more detail see Section 2.1.

There remains a need for a low cost, hand robotic device able to assist from the acute stage of stroke recovery. Such a device must be light-weight and portable, suited for the continuation of rehabilitation in a person's home. The device will need to be attached to the person's hand to enable repetitive exercising of the fingers. The device will need to be capable of both active and passive rehabilitation actions, assisting in completion of hand movements when required. The device will need to also adapt, varying the level of difficultly of the task and the associated movements, as the user improves.

Upper limb spasticity is common following a stroke. If a stiff actuator is used in rehabilitation robotics, undesired motions such as spasms can cause large actuator forces, potentially causing harm to the user [15]. Stiff actuators travel through a fixed trajectory regardless of external forces applied. A compliant actuator is the complement of a stiff actuator. Therapy robots using compliant actuation permit deviations from their equilibrium position, allowing damping of external forces such as hand spasms. Therefore adaptable compliant actuation is required to make rehabilitation robotics safer and more natural.

During an undergraduate project we had previously investigated rehabilitation robotics $[16,17]$. To meet growing rehabilitation demands, the project explored an acute stroke hand rehabilitation device. The purpose of that project was to investigate various rehabilitation ideas and develop a proof of concept. A system was designed to exercise and strengthen an affected hand and was integrated with a basic motivational game. The project gained industrial interest, which led to collaboration with Im-Able Ltd, a company producing upper limb rehabilitation devices. The aim of the wider project is to develop a device that will be incorporated into the 
suite of existing Im-Able products, meeting the current need for a device suited to the acute stroke stage.

As the undergraduate project was only a proof of concept, it was limited in functionality and was not suitable for clinical trials. The system was a fixed, table top design that repetitively opened and closed a person's hand. A magneto-rheological linear piston damper was developed to oppose the user's movements, increasing the difficulty of the exercise. Although the control characteristics of the damper proved promising, problems were identified with both the design of the damper and the use of magneto-rheological fluid. Problems included the settling and leakage of the fluid, and overheating of the housing and fluid. Therefore additional work is required to explore alternative damper designs and to determine the feasibility of the use of magneto-rheological fluid in the rehabilitation device.

\subsection{Scope}

This study is an important stage of a larger research project involving the development of an assistive stroke hand rehabilitation device, which requires a controllable damper to be used in a compliant actuator. The damper is needed to dynamically control the opposing force of the device to vary the level of difficulty of the exercise. This allows the device to adapt as the user improves motor control, continually challenging the user and increasing muscle strength. The damper will also be used to provide compliance to the system. This allows unintended forces caused by the user from spasms, or mechanical noise or oscillations caused by the device to be dampened.

The following outlines the engineering stages required to take a medical device from a conceptual idea through to manufacturing [18].

1. Identify a need

2. Generate ideas to solve the defined problem 
3. Investigate feasibility of concepts

4. Develop a prototype

5. Conduct user testing and clinical trials

6. Reevaluate product

7. Design for manufacture

8. Begin manufacturing

The undergraduate project met the first and second stages of the process and the purpose of this study is to complete the third stage. The presented work studies the use of magneto-rheological fluid to create a controllable damper and investigates the viability of its use in a stroke rehabilitation device. Therefore, the research did not explore or conduct comparative testing of alternative technologies, such as pneumatic, hydraulic or electronic actuation.

Design for manufacture was only a secondary consideration as the emphasis was to determine the feasibility of the use of magneto-rheological fluid.

The study focussed on the macroscopic properties of the magnetorheological fluid, rather than a detailed consideration of the microscopic aspects of magneto-rheological fluid behaviour.

\subsection{Aim}

The aim of the research was to determine whether the use of magnetorheological fluid in a controllable damper is viable for use in a stroke rehabilitation device. This will enable the project partners to decide whether to continue the development of a magneto-rheological damper in a rehabilitation device for people affected by stroke. 


\subsection{Research Objectives}

The following are the primary objectives of the research.

Objective One: Determine quantitative specifications for the magnetorheological damper from qualitative requirements

As the study is part of an ongoing wider stroke rehabilitation project, no specific criteria for functionality were given. To determine practicable boundaries and quantify specifications for the project the following were used:

1. Stroke club meetings and stroke events were attended where rehabilitation needs were gathered through direct communication and observation.

2. Meetings with Sunil Vather, Chief Executive of Im-Able Ltd who develop upper limb stroke rehabilitation devices.

3. Research on stroke recovery and magneto-rheological fluid, from academic literature, patents and existing commercial products.

Below are the specifications for the magneto-rheological damper based on the research.

\section{Opposing force}

The magneto-rheological damper needs to be able to vary its opposing force, allowing the level of difficulty of the exercise to be changed. This variation in force is used to continually challenge the user and increase muscle strength. When the damper is in its off state (no magnetic field applied) the output force should be as close to zero as possible. There was no predefined maximum force specified and so the study involved determining what reasonable force outputs would be. (The purpose of the damper is not to provide excessive strength training and therefore large resistive forces are not required, see Section 3.1.) 


\section{Consistency}

The opposing force produced by the damper needs to be consistent with changes in rotational speed and input current. This will allow health professions to accurately monitor the patient's recovery progress.

\section{Dynamically controllable}

The damper needs to be dynamically controllable to vary the level of difficulty of the exercise during operation. The opposing force should be electronically controllable, so that there is not need to manually modify the setup of the mechanics of the damper. Acceptable variations in force measurements will be based on the damper's controllability. The damper will be considered controllable if there are noticeable distinctions in the measured force by varying the input current and speed of rotation of the damper.

System operating temperature:

The onset of pain for when an object becomes too hot to touch is around $44^{\circ} \mathrm{C}$ [19]. As a safety precaution the exterior temperature of the damper will need to remain below $34^{\circ} \mathrm{C}$, to allow for a margin of error and user comfort.

\section{Power consumption}

As the stroke device is required to be portable, it will be reliant on battery power. Continuous operation is likely to last around an hour, so the power consumption of the damper will need to be considered in the design.

\section{Size}

The stroke device will be designed to sit around a person's arm. Therefore the size of the damper is required to fit within this area, approximately $100 \mathrm{~mm} \times 100 \mathrm{~mm}$. 
Mass

Due to the intended portability of the stroke rehabilitation device the contributing mass of the damper is important. The assumption is made that the total mass of the device will be approximately two kilograms, allowing a stroke survivor to carry the device without assistance. Considering the mass of the housing, motors and other components required for the device, the mass of the damper is required to below $500 \mathrm{~g}$.

\section{Cost of housing}

Magneto-rheological fluid can be expensive, ranging from 480-750 USD for a litre $[20,21]$. For the use of the fluid to be feasible, the damper itself needs to be cost effective. Costs can be reduced through effectively utilising the properties of the fluid and simplifying the damper construction to reduce manufacturing and assembly cost.

Objective Two: Determine the most applicable configuration and mode to use for the magneto-rheological damper

Existing dampers using magneto-rheological fluid fall into several classes, with differing strengths and weaknesses. Research will be carried out to gain an understanding of the properties of magneto-rheological fluid and its main modes of operation (valve, shear and squeeze), to allow informed design decisions to be made (see Section 2.2.3 and Section 3.1). Any disadvantages of the use of magneto-rheological fluid will be assessed so that they can be mitigated or avoided in the design of the damper. Comparisons between existing magneto-rheological dampers will be used to determine the best configuration for our application. 


\section{Objective Three: Design and construct a test rig to measure the magneto- rheological damper performance}

A repeatable and reliable method of acquiring the force produced by the magneto-rheological damper is required. The hardware will need to sample and collect force and temperature measurements as required in Objective One. The physical structure of the test rig will need to be accommodating for the variations in damper sizes due to potential damper design iteration. The data is required to be displayed during measurements, to assist in identifying characteristics of the different damper designs. Data must be able to be recorded for later data analysis.

\section{Objective Four: Design and build a functional magneto-rheological damper to meet the specifications in Objective One}

Following from Objective Two, damper designs will be evaluated and their opposing force measured. The ability for the vane designs to change the generated force of the damper using fluid with known viscosities will be tested before using the designs in magneto-rheological fluid. The performance of each design will be evaluated based on how it meets the specifications in Objective One. Emphasis will be placed on the range of force produced, the consistency of force outputs, and ease of manufacture and assembly. Results will be used to select a particular design and any applicable modifications required will be made to meet additional requirements, such as size and weight.

\section{Overall Objective}

To deem magneto-rheological fluid viability for use in the stroke rehabilitation device the viscous torque, an intrinsic property of the damper reliant on speed, will need to be controllable with varying input current. The required conditions are that the output torque measurements are repeatable and that they occur within the specified operating temperature of the 
system, below $34^{\circ} \mathrm{C}$.

\subsection{Benefits}

The outcomes of this research will be used in a larger project involving the design of a stroke rehabilitation device for an affected hand. If the findings are positive then a magneto-rheological damper will be implemented in the device, otherwise alternative technology will be investigated. However the results of the research could still be extended to other projects requiring controllable damping or similar functionality.

The test rig will be used for the next stages in the stroke rehabilitation project as well as other projects that required force measurements to be made. 


\section{Chapter 2}

\section{Background}

The following chapter describes existing commercial robotic devices used for stroke rehabilitation as well as several systems under development. Magneto-rheological fluid is then introduced and the behaviour of the fluid is explained. The advantages of using magneto-rheological fluid in a rehabilitation device and its challenges are discussed. The different operational modes of the fluid are explained, along with a description of how each mode can be used to create a damper. Current dampers, including both commercial designs and those in development are presented with an emphasis on dampers used in hand applications.

\subsection{Existing Stroke Rehabilitation Devices}

The Able-X [10] device, (Figure 2.1a), by Im-Able, is a passive upper limb rehabilitation device that is suited for home use. The user holds the handlebar shaped device with both hands and their arm movements are used to control specially designed games. The level of difficulty of the exercise is set by the game selected. This device uses bilateral movements to help retrain the affected side, but does not specifically target finger and hand movements.

The Hand Tutor [11], (Figure 2.1b), by MediTouch, is another passive 
device suitable for home use. It uses an ergonomic glove to sense finger and wrist movements which can also be interfaced with games that vary in difficulty. Both these devices only sense the user's movements, they do not initiate or actively assist movements, making them suitable for tracking user progress and for later stages of a stroke once some motor control has been regained.

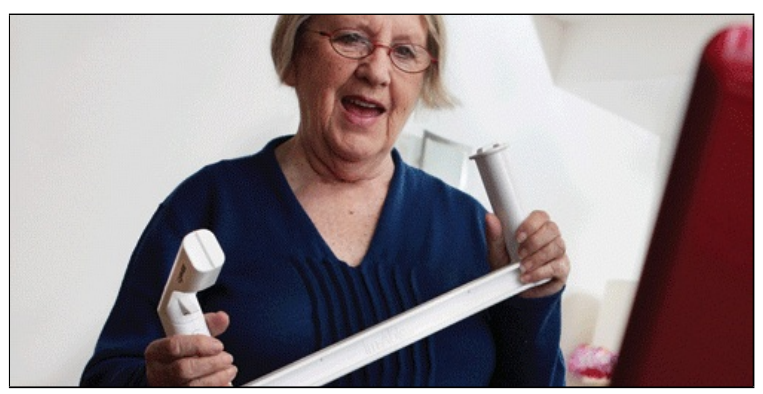

(a)

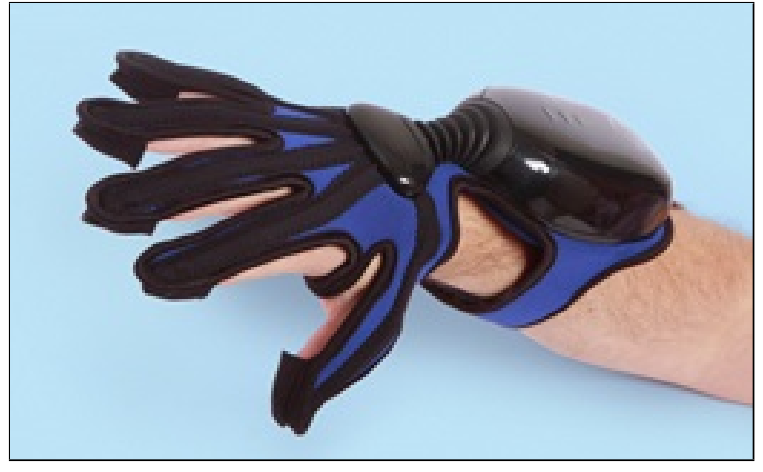

(b)

Figure 2.1: Passive upper limb rehabilitation devices suitable for home use. 2.1a Able-X rehabilitation device, from [10]. 2.1b Hand Tutor rehabilitation device, from [11].

The Amadeo [12], (Figure 2.2), produced by Tyromotion claims to be the only "mechatronic finger rehabilitation device available on the market, that allows each individual finger, including the thumb, to move indepen- 
dently and separately"1 ${ }^{\prime 1}$ The device is able to operate in both passive and active modes and can interface with virtual games. The Amadeo, which retails for over 78,000 USD, is marketed to assist rehabilitation therapists within clinical settings, allowing them to assess the progress of the user. The device is attached to a heavy table to balance the weight of the user's arm, limiting its portability and its set up will often require an additional person (usually the therapist) to help strap the user in.

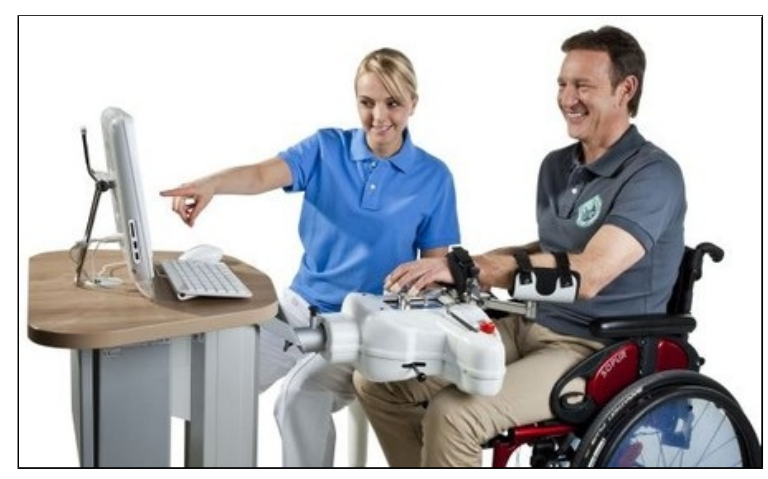

Figure 2.2: Amadeo hand rehabilitation device, from [12]

Rehabilitation devices currently being developed to actively assist hand movements include the HWARD and Gentle/G system. The HWARD (Hand Wrist Assisting Robotic Device) shown in Figure 2.3a, has three degrees of freedom and the pneumatically actuated device assists the hand in grasping and releasing movements [13]. As is the case for the other devices, the HWARD interfaces with a virtual game and does not resist the user's movements. The HWARD is currently undergoing user testing.

Another upper limb device is the Gentle/G (Figure 2.3b) which assists both hand and arm movements to grasp and release objects in a virtual environment. In addition to the device there is an overhead frame that houses a gravity compensation mechanism, to compensate for the weight of the upper arm and forearm [14]. The required space occupied by the system currently makes it unsuitable for most home use.

\footnotetext{
${ }^{1}$ http://www.tyromotion.com/en/products/amadeo/overview.html
} 


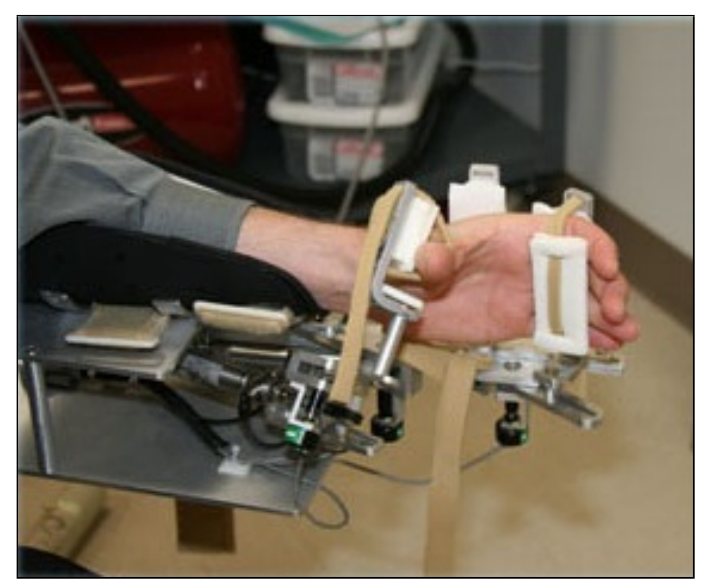

(a)

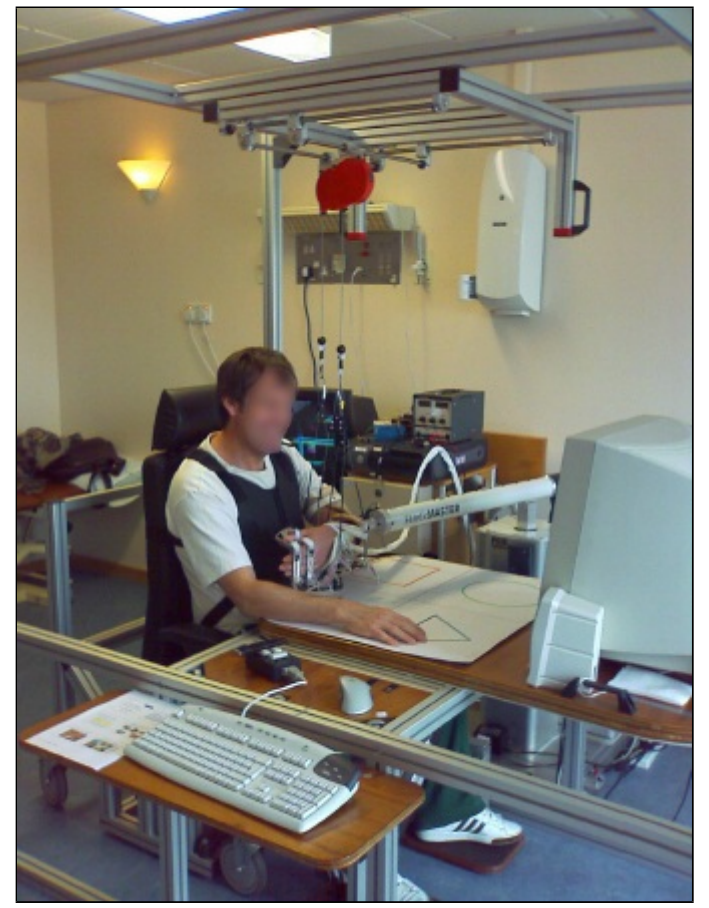

(b)

Figure 2.3: Currently developed active rehabilitation devices. 2.3a HWARD rehabilitation device, from [22]. 2.3b Gentle/G rehabilitation device, from [14]

Although there are a range of robotic devices targeting upper limb re- 
habilitation, there is currently no low cost device, that can both assist and oppose the user's hand movements while being suitable for home use.

\subsection{Magneto-rheological Fluid}

Although discovered in the 1940's by Jacob Rabinow [23], Magnetorheological (MR) fluid for product development only began gathering interest in the 1990's [24]. MR fluid is currently used in a range of applications, including suspension systems, seismic dampers, prosthetics and exercise equipment [25].

MR fluid is classified as a smart fluid as it is able to change its rheological behaviour with changes in the applied magnetic field. The fluid contains iron particles approximately $1-10 \mu \mathrm{m}$ in diameter that are suspended in a liquid (often oil based) [25]. The total solid content of MR fluid ranges from $70-85 \%$ [20, 21]. In the presence of a magnetic field, the iron particles become polarised and align with the field. Opposite poles of the iron particles attract to form chain-like structures parallel to the magnetic field lines, shown in Figure 2.4. 


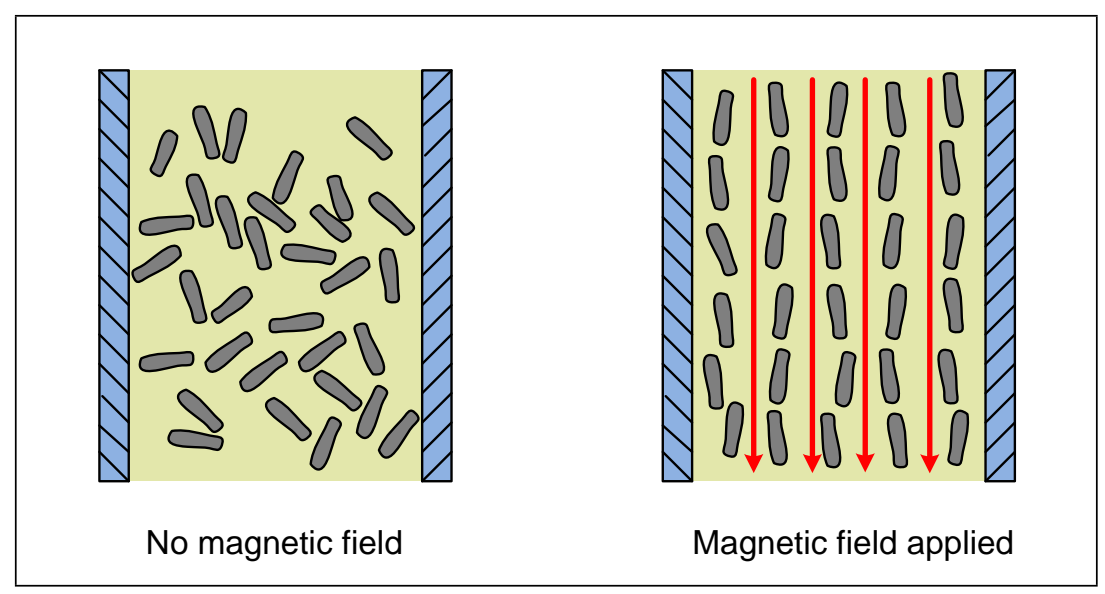

Figure 2.4: MR fluid structural change when a magnetic field (indicated by the arrows) is applied. In the presence of a magnetic field, the iron particles become polarised and form chain-like structures parallel to the field lines.

The change in macroscopic fluid properties is caused by the additional mechanical energy needed to break the chain-like structures, and results in an increase in the viscosity of the fluid. The required mechanical energy increases with increases in field strength, resulting in a field dependent yield strength. However the yield strength is limited and will begin to plateau as the MR fluid approaches magnetic saturation. MR fluid is often modeled as a Bingham plastic having a variable yield strength [24].

\subsubsection{Advantages of MR fluid}

The formation of the chain structures is reversible, allowing the fluid to be magnetised repeatedly. The time taken for the chain structures to form are in the order of milliseconds, in concentrated suspensions and likewise when the field is removed the structure rapidly disappears [25]. This allows the fluid to be dynamically controlled without appreciable lag, which is vital for the safety of the user and operation of the device. The wide range of yield strengths enables smaller, more compact devices to be made 
[26].

The dynamic controllability and fast response time of MR fluid lends it to being well suited for human interactive devices. The intended use of MR fluid in the stroke rehabilitation device, is to provide a variable force to oppose the user's hand movements. The fluid's ability to dynamically vary the opposing force allows it to accommodate for the natural movement of muscles and joints. Biological force is produced by contracting muscles to lengthen and shorten them [27]. When a muscle is very short there are too many overlaps among the filaments to allow much tension build up, and when the muscle is heavily lengthened there is too little overlap to development appreciable tension. Maximum force is generated when a joint is in the middle of its range of motion, when the muscles are at their intermediate length [28]. Therefore the fast, dynamic controllability of MR fluid is advantageous as it can vary the opposing force applied with respect to the joint's position in the range of motion. Also MR fluid is inherently safe, as it only applies passive forces during movement and the ability to vary the opposing force also allows the system to adapt for multiple users.

\subsubsection{Challenges of MR fluid}

A challenge with using MR fluid is its high cost, which ranges from 480750 USD per litre. Currently there are only two companies who commercially sell MR fluid; LORD Corporation and Liquids Research Limited. As interest in the use of MR fluid increases each year, it is possible that the growing demand may cause a reduction in price. Until this reduction occurs the MR fluid will need to be judiciously utilised, to simplify and reduce the manufacturing and assembly costs of the housing.

A practical challenge in using MR fluid is that the iron particles tend to settle. The settling of the iron changes the viscosity of the different areas of the fluid, which will effect the controllability of the fluid. This is a 
problem in devices that are seldom used but are required to perform instantly when required, such as seismic dampers. A possible approach to reduce this effect is to weakly aggregate the fluid but this would increase the complexity of the damper and could affect the viscosity response of the fluid [25]. Another approach is to use a non-newtonian medium [29] or stabilising agents such as lithium grease [27]. Although not immune to particle settling, advancements have been made in commercial MR fluid to increase the particles resistance to hard settling and aid redispersability [20]. Precautions will need to be taken to ensure the design of the damper promotes iron particle suspension during operation and easy recombination of particles if settled.

Due to the high iron concentration and use of an electromagnet, the size and mass of the damper could become large. This increases the complexity of designing compact, portable devices containing MR fluid.

The quality of the iron particles and carrier fluid used cause MR fluid to be expensive ranging from 480-750 USD for a litre [20, 21].

Magnetic hysteresis may also be a problem, with some iron particle remaining magnetised after the magnetic field has been removed. Heating the fluid or reversing the field can reduce the effects. However, heating the fluid can cause changes in the viscosity of the carrier fluid counteracting the alignment of the iron particles [16].

\subsubsection{Operational modes of MR fluid}

The operational modes of the fluid were explored to understand how the rheology properties of MR fluid are best utilised to achieve the required damping. The three common operational modes of MR fluid, are valve mode, direct shear mode and squeeze mode. 


\section{Valve (Pressure) mode}

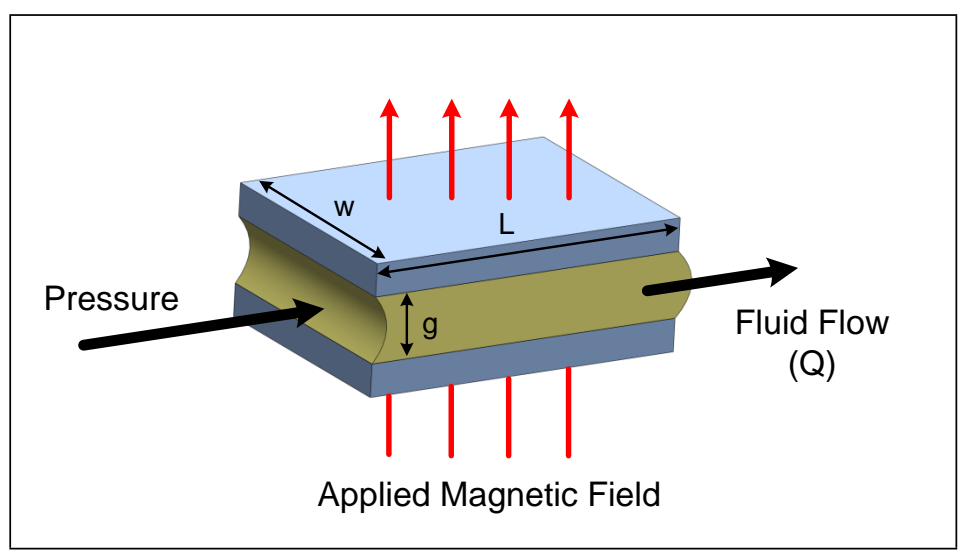

Figure 2.5: Valve (Pressure) operational mode of MR fluid

In valve mode (Figure 2.5) the MR fluid flows from one chamber to another through a channel between two fixed plates (poles). A magnetic field is applied perpendicular to this flow. In the active region (the channel) the viscosity of the fluid is increased, becoming like a solid. No flow occurs in the active region, until the pressure build up is sufficiently large to yield the solidified fluid, pushing it through the channel [30]. The yield strength can be increased by increasing the strength of the magnetic field. The valve mode is the most widely used of the three modes and is often used in shock absorbers. Dampers using this mode can provide large displacements and can generate forces up to several hundred of newtons [31].

The pressure drop in a damper containing the valve mode is comprised of the sum of the viscous component $\Delta P_{n}$ and a magnetic field dependent induced yield stress component $\Delta P_{\tau}$. The pressure is approximated by [24]:

$$
\Delta P=\Delta P_{n}+\Delta P_{\tau}(H)=\frac{12 \eta Q L}{g^{3} w}+\frac{c \tau_{y}(H) L}{g}
$$

where $L, g$ and $w$ are the length, gap and width dimensions of the channel, $Q$ is the volumetric flow rate, $\eta$ is the viscosity of the fluid with no 
field applied, $\tau_{y}$ is the yield stress developed in response to the applied magnetic field intensity $H$ and $c$ is a function of the flow velocity profile ranging from 2-3 depending on a corresponding ratio of $\frac{\Delta P_{\tau}}{\Delta P_{n}}$ (from 1-100).

As shown in Equation 2.1 a long narrow channel with reduced gap would result in a larger force output of the damper, compared with short, wide designs.

\section{Direct Shear mode}

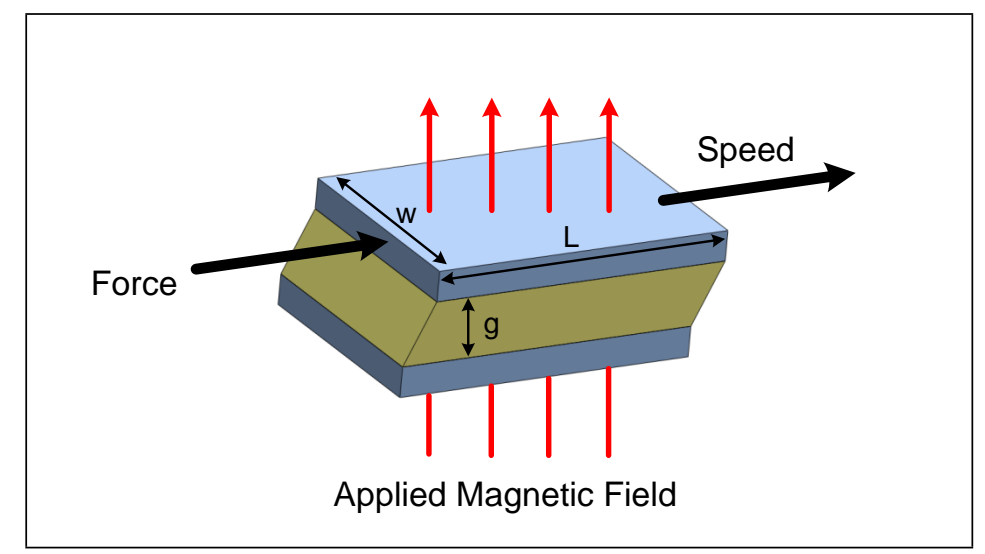

Figure 2.6: Direct shear operational mode of MR fluid

In shear mode (Figure 2.6) the MR fluid is contained between two parallel plates (poles) and a magnetic field is applied perpendicular to the poles. One pole is fixed while a force is applied to the other pole causing it to rotate or move parallel relative to the fixed pole. Resistance is cause by the pole shearing the fluid layer, breaking the chain structure. Increasing the strength of the magnetic field increases the force required to move the pole. Devices that use the direct shear mode are often rotary mechanisms such as clutches and brakes, where medium displacement and force is required.

Similarly to Equation 2.1, the following equation approximates the force 
produced by a direct shear damper [24]:

$$
F=F_{n}+F_{\tau}(H)=\frac{\eta S A}{g}+\tau_{y}(H) A
$$

where $S$ is the speed of the moving pole and $A=L \times w$. In reference to the geometry, Equation 2.2 shows that a reduced gap and large area of the shear pole will increase the total force.

\section{Squeeze mode}

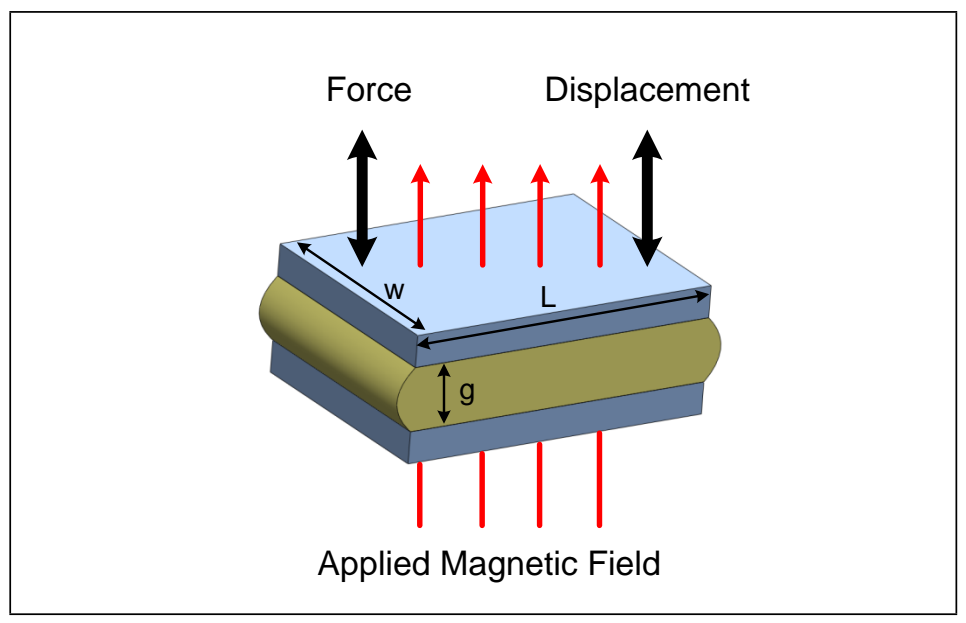

Figure 2.7: Squeeze operational mode of MR fluid

In squeeze mode (Figure 2.7) the MR fluid is contained between two parallel plates (poles) and a magnetic field is applied perpendicular to the poles. Unlike the direct shear mode, a force is applied parallel to the magnetic field to either one or both of the poles causing them to move towards each other. For a specified force value the displacement is caused by the strength of the magnetic field. The displacement amplitudes are often small, in the order of millimetres and the resistive forces are high but non-linear $[27,31]$. The use of the squeeze mode has been investigated in small amplitude vibration and high force impact dampers [32]. 
In the stroke rehabilitation device, the damper will need a displacement range in the order of centimetres to correspond with hand and finger movements. Therefore the millimetre displacement amplitudes of the squeeze mode will not be sufficient for this application. Only the valve mode and the direct shear mode will be further investigated.

\subsection{Applications of MR Fluid}

MR dampers can be classified as linear or rotary. This section focuses on a common linear damper design and a common rotary damper design, and their associated benefits. Existing dampers, both commercial and in development, that are specifically targeted towards hand and wrist applications will be presented. An appreciation will be gained for the current problems faced by these dampers and the magnitude of the force and torque that can be expected. The findings will be used to determine the design of the damper to be used in the stroke rehabilitation device, see section 3.1.

\subsubsection{Linear damper}

The most common linear damper configuration is shown in Figure 2.8, which uses the valve operational mode of the MR fluid. Chambers are formed on either side of the piston and fluid is forced to move through the channels from one chamber to the other, as the piston moves. When the electromagnet is energised, the iron particles are aligned perpendicular to the displacement of the shaft, opposing its movement. 


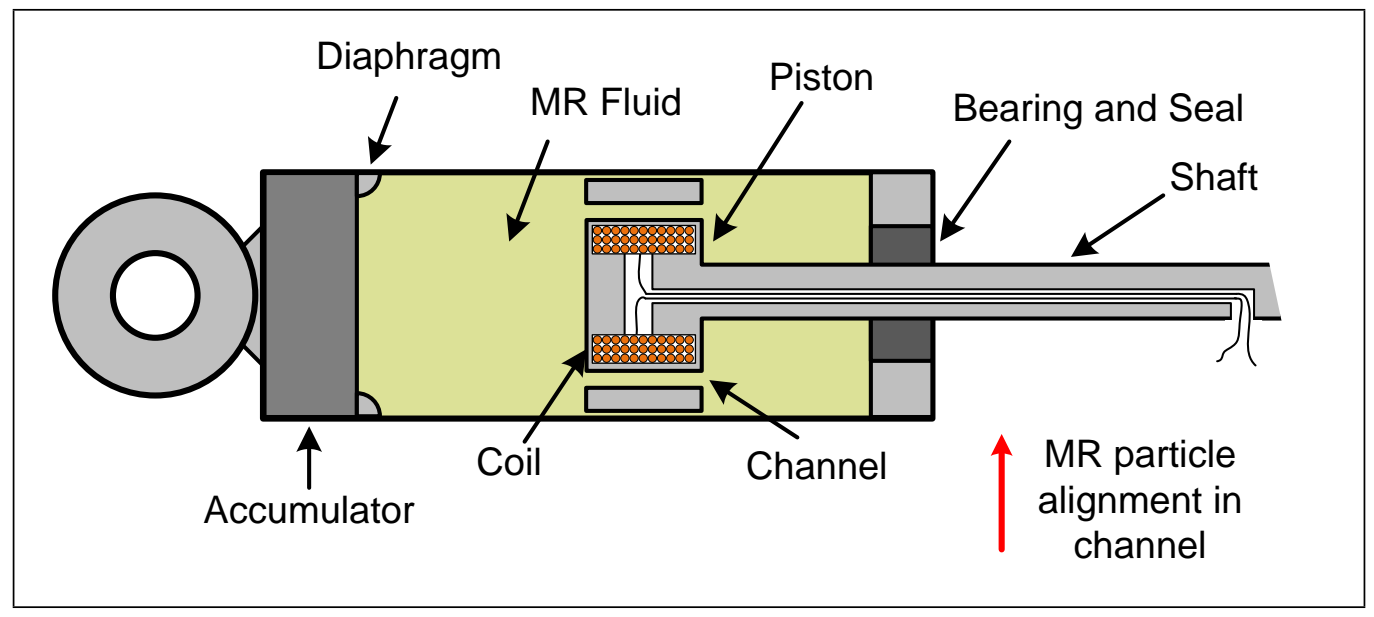

Figure 2.8: Common construction of a linear damper, using the valve operating mode of the MR fluid (adapted from [27])

The ability to provide large forces and displacements makes linear dampers well suited for the automotive industry. Examples of MR dampers include the LORD Motion Master for seat suspension and MagneRide system for car suspension. Current commercial standalone dampers include the LORD RD-8040-1 (short stroke) and the LORD RD8041-1 (long stroke) both retailing for 400 USD. These dampers are designed for industrial suspension applications and are able to withstand nearly $2000 \mathrm{~N}$ at 1 A continuously for 30 seconds or intermittently at 2 A. In the damper's off-state, where no current and field is applied to the fluid, the force required to move the piston in the damper is still large at hundreds of newtons.

Smaller scale linear dampers have also been used in devices to oppose hand and finger movements. A linear actuator containing MR fluid for a force feedback glove system named MRAGES [33], was able to provide a maximum force of $6 \mathrm{~N}$ per finger with an operational current of $0.33 \mathrm{~A}$. However due to the friction between the shaft and seal, in the off-state the output force ranged from $1.4 \mathrm{~N}$ to $1.9 \mathrm{~N}$, for typical finger motions, which causes finger fatigue. In another hand haptic project, the desired force to 
oppose finger movements was $12 \mathrm{~N}$, but due to magnetic saturation $7.5 \mathrm{~N}$ was achieved with 0.5A [31]. This research also had problems with the off-state frictional forces caused by the shaft and seal.

In the undergraduate work prior to this research the off-state force was reduced by using a secondary coil in place of a seal. The coil produced a magnetic field to increase the viscosity of the escaping fluid, thereby reducing its velocity [16]. The magnetised fluid clogged the opening to make it harder for additional fluid to leak while still allowing the shaft to easily pass. The active seal was able to reduce the leakage, but not completely contain the MR fluid.

\subsubsection{Rotary damper}

The direct shear operational mode is commonly used in rotary dampers shown in Figure 2.9. In this study rotary brakes will be referred to as rotary dampers. The shaft is connected to a disc which rotates between two fixed plates. When the electromagnet is energised, the iron particles are aligned parallel to the shaft and as the disc rotates it shears the fluid. The size of the damper and the number of discs used can be varied to suit the application. 


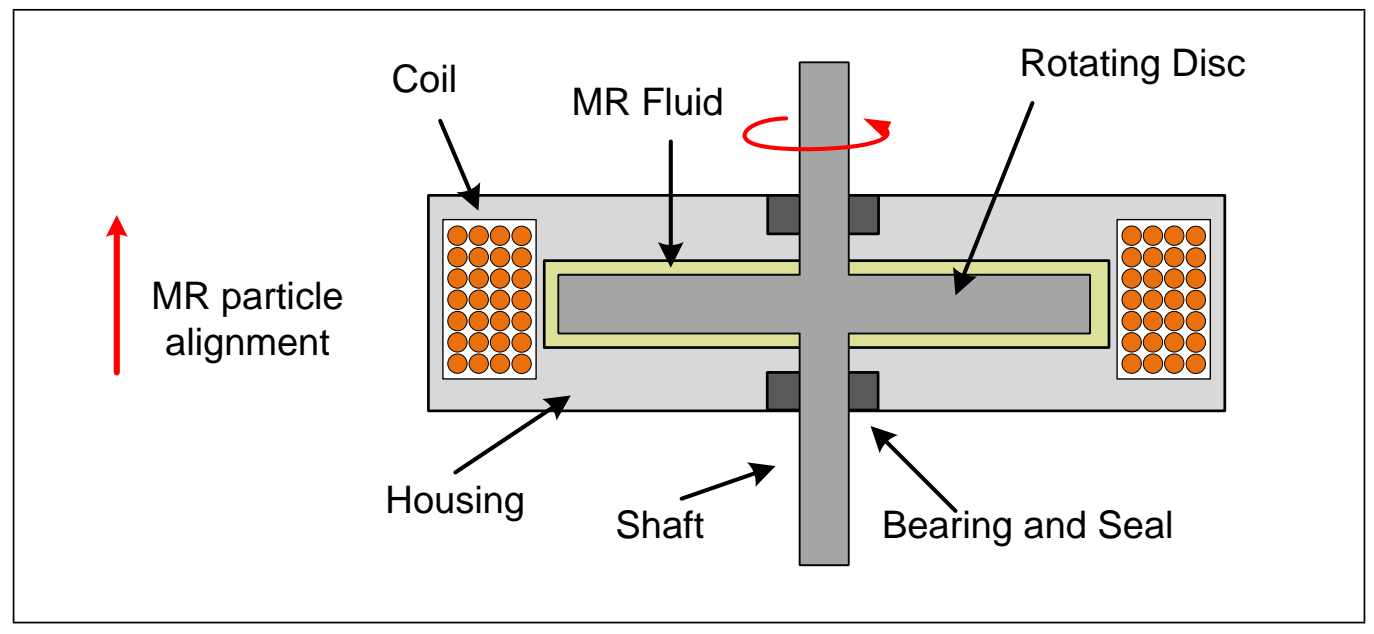

Figure 2.9: Common construction of a rotary damper, using the shear operating mode of the MR fluid (adapted from [27])

In comparison with linear dampers, rotary dampers can be quite compact and therefore have lower power ratings. A commercial rotary damper is the Rheo Knee, which was developed by MIT and commercialised by Ossur. The Rheo Knee is a prosthetic knee with very small fluid gaps of $20 \mu \mathrm{m}$ allowing multiple discs to be used to increase the output torque [34]. The knee contains a control unit that is able to learn and adapt to the user's movements. During operation, the damper is able to provide $40 \mathrm{Nm}$ with only a $60 \mathrm{~mm}$ diameter housing[27] and has an off-state torque of $0.5 \mathrm{Nm}$ [34].

Current developments in hand orientated devices containing rotary dampers include a portable wrist rehabilitation device [35]. This device has a width of $145 \mathrm{~mm}$ and during operation is able to supply a torque of $22.5 \mathrm{Nm}$, with current varying from 0 to $1 \mathrm{~A}$ and in off-state there is a torque of $0.4 \mathrm{Nm}$. Rotary dampers are also being developed for joysticks in haptic devices [36]. In operation the damper was able to supply a torque of approximately $7 \mathrm{Nm}$, with a width of $78 \mathrm{~mm}$ and MR fluid gap of $1 \mathrm{~mm}$. Both devices exhibited hysteresis, with the wrist rehabilitation device having an off-state remanent torque of $2 \mathrm{Nm}$. Although these rotary dampers 
are compact, there is a trade-off between having a large torque range and having a low off-state torque.

\subsection{Summary}

There are several commercial robotic devices and several devices under development that target upper limb rehabilitation. Commercial devices tend to operate passively, requiring the user to control their own movements, whilst devices under development focus on actively assisting the user's movements. One commercial device that is able to operate both passively and actively, is large and expensive and tailored for use in rehabilitation clinics. There remains a need for a low cost device that can both assist and oppose the user's hand movements.

MR fluid has only recently been used in product development. The viscosity of the fluid is dependent on the strength of the magnetic field applied. Advantages of MR fluid include its dynamic controllability and fast response time. Disadvantages of using MR fluid include its cost and the settling of the iron particles. There are three main operational modes of MR fluid, the valve mode, shear mode and squeeze mode, where the squeeze mode is the least used. The valve mode is the most commonly used and is often implemented in linear dampers, while the shear mode is used in rotary dampers. 


\section{Chapter 3}

\section{Method}

The following chapter details the selection of the damper configuration to be used and discusses the design and construction of the test rig. Using the objectives outlined in Section 1.4, the linear and rotary damper configurations were compared and a rotary damper was selected. The design and construction of the damper housing and three vanes are also presented. The vane designs were layered discs, a paddle and a helix. The operation of the test rig and the data analysis procedure are discussed.

\subsection{Selection of Damper Configuration}

Current commercial MR dampers are expensive and are built for large scale applications that need to generate large forces or torques. To make a rehabilitation device that is suitable for home use, the required damper for this application needed to be sensitive to hand movements and cheap to construct. Such a damper is not commercially available, so had to be designed and built. Selection criteria based on Objective One were used to determine whether a linear or rotary damper was to be used. 


\subsubsection{Force requirements}

The resolution of the required force is taken from the just noticeable difference (JNDs) force with regards to finger movements in healthy individuals. This difference is approximately $7-10 \%[37,38]$. Although the JND for a person who has had a stroke may differ to a healthy individual (most likely higher) the resolution in force control of the damper will be set for a healthy individual.

In the on-state (current supplied), the damper will need to produce sufficient opposing force to challenge and stimulate the user. Based on similar sized dampers used in hand haptic devices, the required output force will range between approximately 0 and $20 \mathrm{~N}$, which both linear and rotary dampers are capable of supplying. The important aspect of the device will be its ability to be back-driven, allowing users to control their own movement when the device is passive and the damper is in its offstate. Therefore the output force of the damper in its off-state is required to be as close to zero as possible to reduce fatiguing during the exercise.

The problem with using linear dampers for sensitive applications is the trade-off between using a tight seal to prevent fluid leakage and the frictional force between the shaft and seal resulting in an unwanted 'offset' force, which becomes a problem in the off-state. Unlike linear dampers, rotary dampers are able to use bearings to minimise the off-state torque and leakage. The large off-state torques observed in the rotary designs mentioned in Section 2.3.2, are often due to the increased number of discs and the reduced size of the fluid gaps to allow larger output torques to be generated. As large torques are not required in this application this is not expected to be a problem.

\subsubsection{System operating temperature}

As the temperature of the carrier fluid increases its viscosity will begin to decrease, which will tend to counteract the iron particle alignment [16]. 
Therefore the increased temperature of the fluid could be an issue if the damper is active for a long period of time. Because of this effect the LORD Corporation dampers are only rated to work for 30 seconds continually. As most oil and silicon based fluids operate from -40 to $150^{\circ} \mathrm{C}$ and water based fluids are rated from 0 to $70^{\circ} \mathrm{C}$, it is important that the temperature of the damper does not exceed these ranges. The advantage of the linear damper is that the field is only applied to the necessary region of fluid and during operation this region changes allowing heat to be distributed. In a rotary damper all the fluid is magnetised at the same time, which can cause heating issues if used over a long time period. This can also increase the chances of magnetic hysteresis.

\subsubsection{Size, mass and power consumption}

As the length of a linear damper is determined by the displacement required, there is limited flexibility in reducing the total size of the damper. The nature of the rotary damper allows it to be reduced in size and weight making it suitable for use in a portable device. The vane is able to rotate irrespective of the required displacement, allowing the damper to accommodate varying hand sizes and movements. The existing dampers discussed in Chapter 2 were all rated to operate around 1 to $2 \mathrm{~A}$, which is practical for a low-power device aiding portability.

\subsubsection{Selected configuration}

After comparing the linear and rotary dampers against the required objectives, the rotary damper was chosen. Although rotary dampers are not conventionally used in linear displacement applications, the device can benefit from the compactness in design and the reduced output torque in the off-state of the damper. 


\subsection{Test Rig Designs to Measure Force}

A test rig was needed to quantify the performance of the MR damper design. The test rig was required to be durable as multiple trials were necessary to ensure reliable force measurements. It was also important that the intrinsic properties of the test rig neither assisted nor hindered the damper's operation. The opposing force of the damper needed to be measured for a range of linear speeds and input currents.

A pulley was used to convert the damper's rotational motion to linear motion. Pulleys provide smooth motion and are easy to implement. They are also flexible as the number of damper shaft rotations per cycle could be increased or decreased by changing the size of the pulley used.

The following section discusses two design approaches used to measure the opposing force of the damper. A mock-up of each design was made to test the functionality and suitability of the design for the test rig. A linear test rig was built first, however as it was not able to meet the required specifications a pulley system was developed.

\subsubsection{Linear test rig}

A linear test rig was built to measure the opposing force of the rotary damper as shown in Figure 3.1. A DC motor and pulley were used to pull the force sensor (see Section 3.3.1) at a constant linear speed. The other end of the sensor was connected to the damper's pulley. During operation the force sensor was pulled across the beam at a constant speed, unwinding the damper's pulley. As it was pulled, the sensor measured the opposing force generated in the damper.

The motor's speed was calibrated with the force sensor attached, so that the mass of the sensor did not contaminate the results. The time taken for the force sensor to move $500 \mathrm{~mm}$ with varying input voltages was measured using a stopwatch. The input voltages ranged between 2-11 V and three trials at each voltage level were taken. 


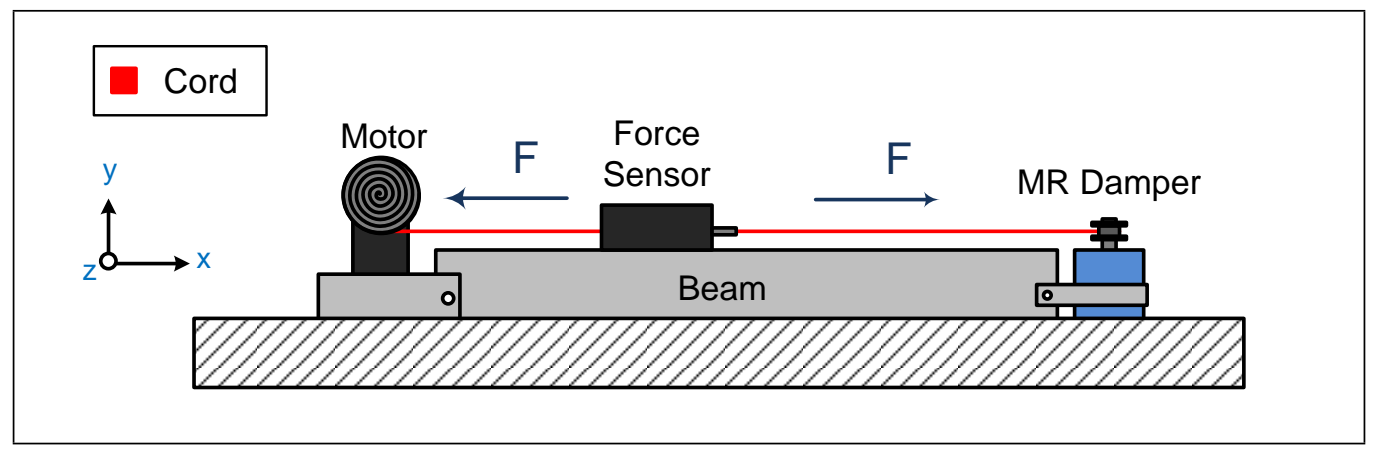

Figure 3.1: The linear test rig used to measure the opposing force of the magneto-rheological damper. A force sensor was located between the DC motor and the damper. The motor was used to pull the force sensor across the beam at a constant linear speed. As the sensor was pulled it measured the opposing force generated by the damper.

Referring to Figure 3.1 the force sensor was pulled in the negative $x$ direction. Any movement in the $\mathrm{z}$ direction would cause inaccurate readings as the measurements assume that the cord is pulled tangential to the pulleys. Therefore walls were built on either side of the beam to guide the sensor and restrict movement in the $z$ direction, see Figure 3.2. There was a small gap on either side of the sensor to prevent additional friction from the sensor rubbing against the walls.

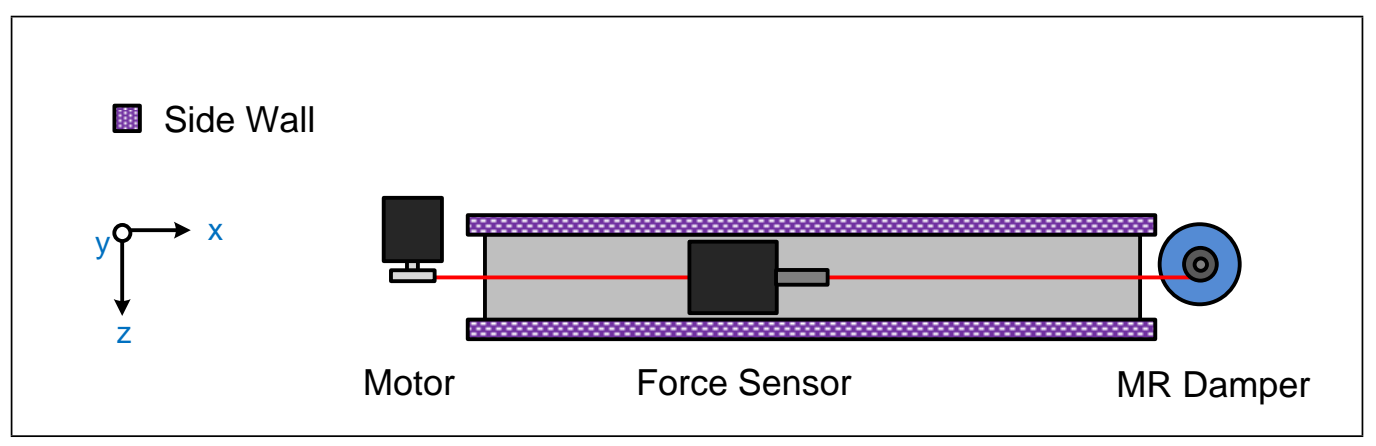

Figure 3.2: Top view of the linear setup of the test rig. The walls on the sides of the force sensor were used to limit the $\mathrm{z}$ direction movement of the sensor. 
However, it was found that the sensor would occasionally contact the wall causing an increase in the force measurements. Also when the force sensor was pulled along the beam at low speeds, the additional force introduced by the friction between the sensor and the beam was substantial in comparison to the opposing force of the damper.

An additional disadvantage of the linear setup was its lack of flexibility. The number of times the damper's shaft could be rotated was determined by the length of the beam. As the beam's length needed to be chosen before testing and damper iterations began, there was the possibility that for certain designs the beam may not be long enough to provide adequate testing.

The linear system showed that the measured forces of the damper were sensitive to the intrinsic frictional forces of the test rig. As the linear system was unable to produce reliable and repeatable results a lower friction system was needed.

\subsubsection{Pulley test rig}

A reduced friction test rig was designed with the use of pulleys. The concept of the pulley system is shown in Figure 3.3. The motor rotated at a constant angular speed and unwound the pulley attached to the damper to rotate the shaft. A floating pulley, $\mathrm{P}_{1}$, was attached to the force sensor. During operation, as the damper opposed the turning of its shaft, $\mathrm{P}_{1}$ was pulled down in the y direction, changing the measured force. The configuration of the system meant that the force measured was double the output force of the damper [39]. The fixed second pulley, $\mathrm{P}_{2}$, was used to ensure that the system was tangentially unwinding the damper's pulley to maximise the torque generated by the damper. 


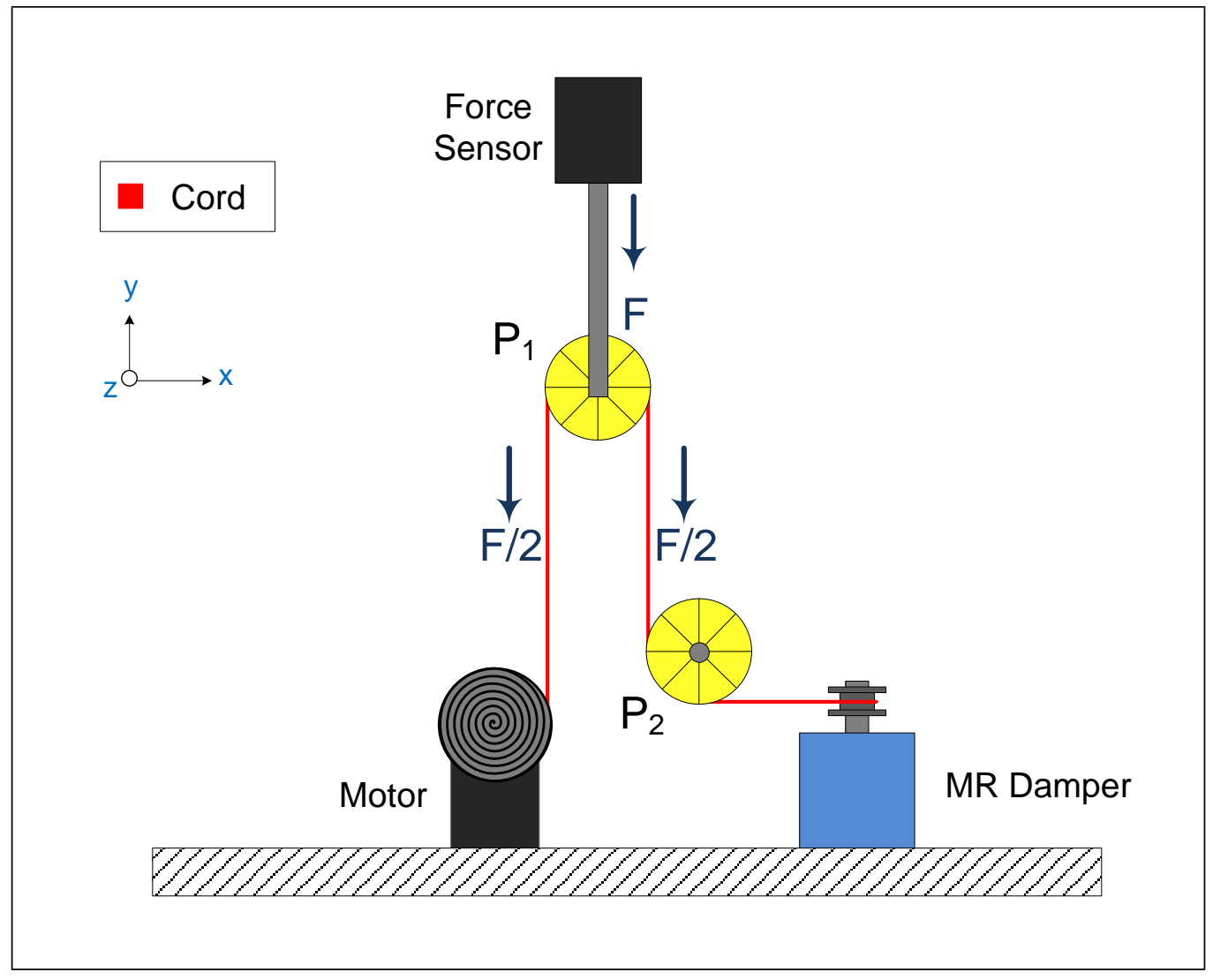

Figure 3.3: The pulley test rig used to measure the opposing force of the magneto-rheological damper. The motor rotated at a constant angular speed and through the pulley system it rotated the damper's shaft. Pulley $\mathrm{P}_{1}$ was attached to a force sensor, and pulley $\mathrm{P}_{2}$ was fixed. During operation the opposing force of the damper pulled $\mathrm{P}_{1}$ vertically down, changing the measured force. The configuration of the pulley caused the force sensor to measure double the output damper force.

Ball bearings were used to reduce the friction caused by the rotation of the pulleys. The advantage of the pulley configuration was that the friction forces in the system were relatively small in comparison to the opposing force of the damper. The disadvantage of the design was that the components needed to be aligned precisely to ensure the results were 
accurate and repeatable.

The initial pulley test rig was set up using retort stands and clamps to demonstrate the functionality of the design. After the test rig was tested and deemed suitable for this application a permanent frame was designed and built, as shown in Figure 3.8.

\subsection{Component Selection and Final Test Rig Con- struction}

The setup of the test rig needed to be flexible to accommodate for potential damper design iteration. The availability of an extruding 3D printer that had an accuracy of $0.4 \mathrm{~mm}$, allowed some parts to be designed in SolidWorks and printed in PLA.

\subsubsection{Force and temperature component selection}

Components for the test rig were chosen based on functionality, availability and suitability for PC interfacing.

As discussed in Section 3.1, the output force range of the MR damper was approximated to be $0-20 \mathrm{~N}$. The setup of the pulley test rig meant that the force sensor needed to measure forces at least double the required force range, therefore having a minimum range of 0-40 N. A Vernier DualRange Force Sensor ${ }^{1}$ was used to measure the opposing force of the MR damper as its force range was rated from $-50 \mathrm{~N}$ to $+50 \mathrm{~N}$ with a precision of $0.05 \mathrm{~N}$. A Vernier LabPro Data Collector ${ }^{2}$ was used to interface the force sensor with the PC.

As required in Objective One (Section 1.4), the temperature of the MR damper needed to be monitored to ensure a safe level of operation and to

\footnotetext{
${ }^{1} \mathrm{http}: / /$ www.vernier.com/products/sensors/force-sensors/dfs-bta/

${ }^{2} \mathrm{http}: / /$ www.vernier.com/products/interfaces/labpro/
} 
investigate behavioural changes in MR fluid due to heat. A LM35 $35^{3}$ temperature sensor IC was chosen due to its low cost. The LM35 output voltage is linearly proportional to changes in temperature and it has a temperature range of $-55^{\circ} \mathrm{C}$ to $150^{\circ} \mathrm{C}$ with accuracy of $0.25^{\circ} \mathrm{C}$. Some experimentation required an additional LM35 to be submerged in various fluids (see Section 4.2), therefore the temperature sensor was waterproofed. A cross section of the temperature sensor is shown in Figure 3.4.

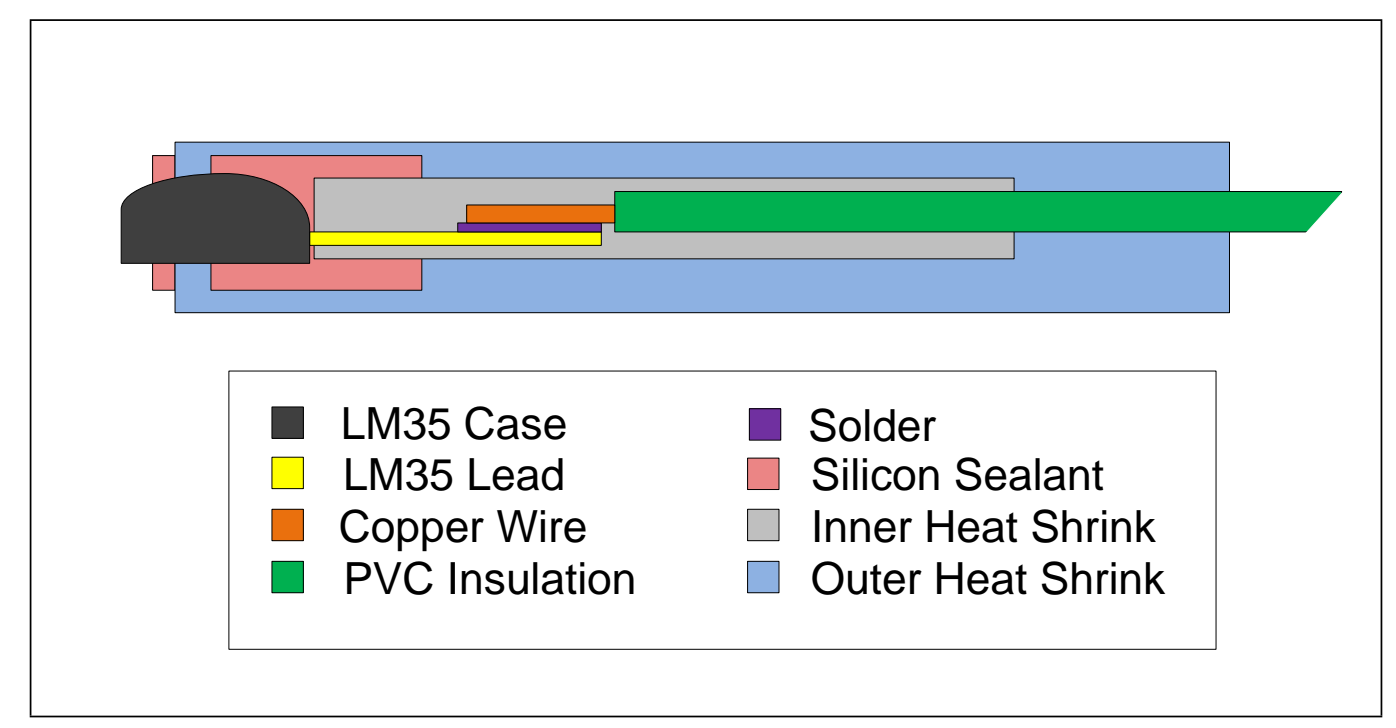

Figure 3.4: Cross section of the LM35 temperature sensor showing the layering of the various materials used to waterproof the sensor.

The process of insulating the temperature sensor was as follows: The leads of the LM35 were soldered to three wires; voltage supply, ground and the voltage output. The inner heat shrink was used to hold each connection in place and insulate the leads from each other. Silicone sealant was then used to insulate the exposed leads. The outer heat shrink was used to hold the silicone sealant in place. To prevent the temperature readings from being distorted due to additional material layers, a part of the

\footnotetext{
${ }^{3}$ https://www.national.com/ds/LM/LM35.pdf
} 
LM35 casing was left exposed. To further prevent fluid from seeping in, silicone sealant was again used on the outside.

A National Instruments 6009 Data Acquisition Board ${ }^{4}$ (DAQ) was used to power the temperature sensor and interface it with the PC. The LM35 sensor was calibrated against a thermometer measuring the temperature of water that ranged from $10^{\circ} \mathrm{C}$ to $85^{\circ} \mathrm{C}$, which is within the specified temperature range in Objective One. Using the DAQ in RSE (referenced single ended) mode, it was found through the calibration process that the output voltage of the LM35 saturated for temperatures below $31^{\circ} \mathrm{C}$. At temperatures below $31^{\circ} \mathrm{C}$ the internal configuration of the DAQ required the LM35 to sink current, which it was not able to do. To solve the problem a $220 \Omega$ was placed between the output terminal of the LM35 and ground, which provided a path for the current to flow to ground.

\subsubsection{Cord selection}

The test rig used braided nylon cord as it does not stretch easily, therefore when wound around the damper's pulley it was able to hold its position without assistance. Also the strength and weaving of the rope allowed multiple trials to be conducted without the rope needing to be replaced.

\subsubsection{MR damper pulley and shaft}

The damper's shaft and pulley were made from metal as they needed to withstand repetitive measurements. Stainless steel was used as it is nonmagnetic. To allow flexibility for different sized pulleys and shafts for later stages of the research, the shaft and pulley were not fixed, instead the pulley screwed onto the top of the shaft, shown in Figure 3.5. A $6 \mathrm{~mm}$ shaft was used to minimise the volume it occupied inside the damper, and still be able to withstand the anticipated forces of $0-20 \mathrm{~N}$. The radius of the pulley was reduced in width to maximise the measured output force range

${ }^{4}$ http://sine.ni.com/nips/cds/view/p/lang/en/nid/201987 
of the MR damper for greater precision. As the diameter of the shaft was $6 \mathrm{~mm}$, accounting for a suitable wall thickness, the diameter of the pulley was $8 \mathrm{~mm}$.

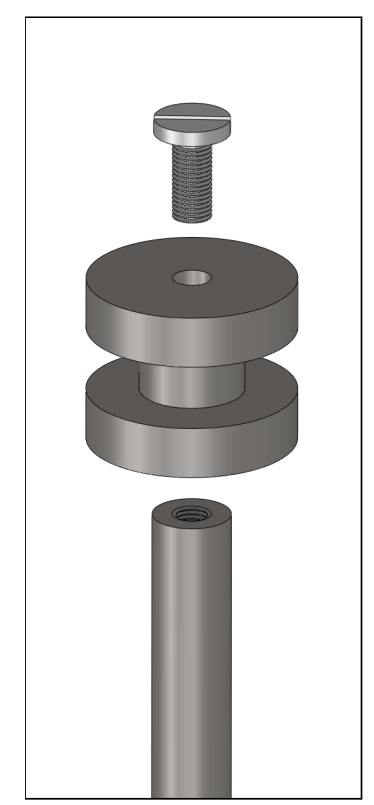

(a)

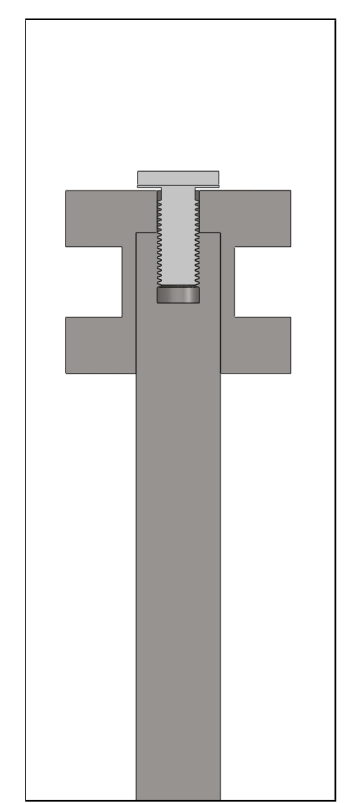

(b)

Figure 3.5: Damper's pulley and shaft. 3.5a Exploded view of the the pulley attachment to the shaft. $3.5 \mathrm{~b}$ Cross-sectional view of the pulley and shaft

\subsubsection{Test rig pulleys}

Pulleys $\mathrm{P}_{1}$ and $\mathrm{P}_{2}$ used in the test rig contained ball bearings to reduce rotational friction. Shown in Figure 3.3, pulley $\mathrm{P}_{1}$ needed to be attached to the force sensor. As $\mathrm{P}_{1}$ needed to be suspended but not fixed, a housing for the pulley (see Figure 3.6) was made to attach the pulley to the force sensor. The connection between the pulley housing and the force sensor was rigid to minimise movements of the pulley in the $\mathrm{x}$ and $\mathrm{z}$ directions (see Figure 3.3), which would have caused inaccurate force measurements. The pulley cover also contained a ridge to guide the cord and prevent it 
from slipping off the pulley, (which was previously common at the end of a test run when there was slack in the cord). The pulley and the cover were connected by a shaft and spacers were placed on either side of the pulley to keep it centred.

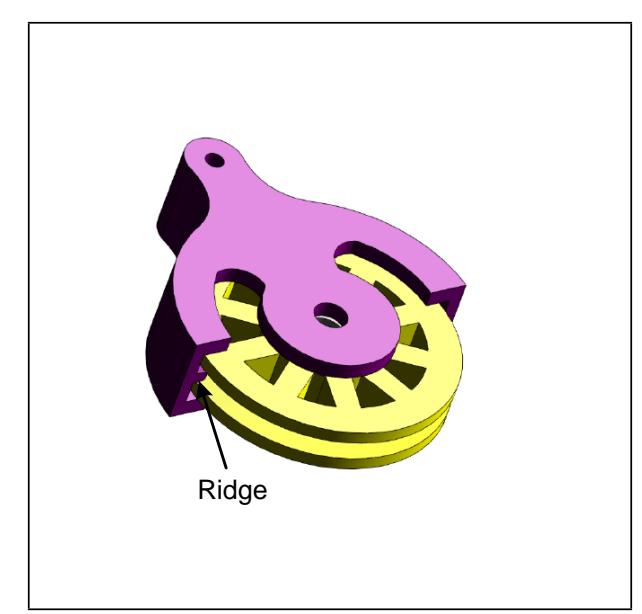

(a)

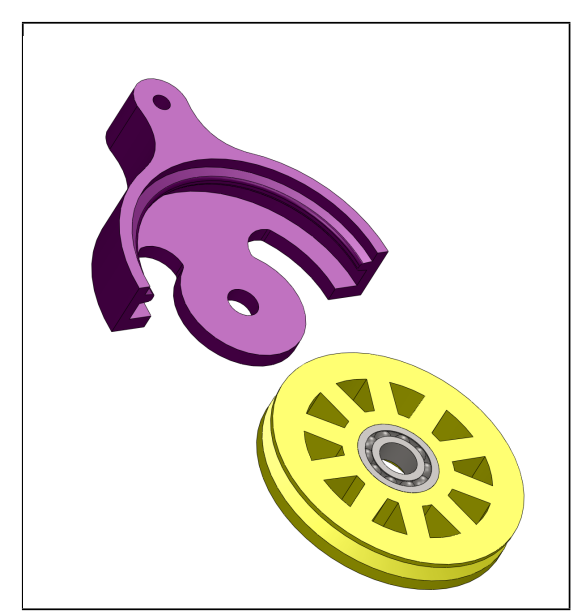

(b)

Figure 3.6: SolidWorks render of the pulley cover used to attach pulley $\mathrm{P}_{1}$ to the force sensor. 3.6a SolidWorks render showing the placement of the pulley inside the pulley cover. The shaft and washers used to connect pulley and cover are not shown. 3.6b Exploded view showing the ridge in the pulley cover used to guide the cord and the ball bearing in the pulley, used to reduce rotational friction.

The pulley cover was $3 \mathrm{D}$ printed due to its complexity and pulleys $\mathrm{P}_{1}$ and $\mathrm{P}_{2}$ were also printed. The layering printing technique used required support structures to be printed for the cover and pulleys, which were later removed from the finished pieces. Due to the support structure and the printing technique, a large amount of filing was needed to prevent the cord from catching on the rough surfaces. Also as the printer struggled to print round holes, pilot holes were printed which were later drilled to the correct size. 


\subsubsection{Motor considerations}

To improve the control of the system, the DC motor was replaced by a servo motor. The use of a servo motor allowed the PC to accurately control the speed and position of the motor's movements. As servo motors have a limited angle range, a pulley was attached to the motor. This pulley increased the distance the cord could be pulled, which correlated to an increase in the number of rotations of the damper's shaft.

To evaluate the force output for the damper design and observe its characteristics, a minimum of five rotations of the shaft were required. As the damper's pulley had a radius that ranged from $4-8 \mathrm{~mm}$, an averaged radius of $6 \mathrm{~mm}$ was used to determine the minimum linear displacement required to rotate the damper at least five times. The calculation is as follows:

$$
\begin{gathered}
2 \times \pi \times r_{p}=37.7 \mathrm{~mm}\left(\text { where } r_{p}=6 \mathrm{~mm}\right) \\
37.7 \times 5 \text { rotations }=189 \mathrm{~mm}
\end{gathered}
$$

Therefore the pulley attached to the motor needed to move a minimum translated linear distance of $189 \mathrm{~mm}$. A servo motor with an operating angle of $180^{\circ}$ would require a pulley attachment with a radius of:

$$
\begin{gathered}
2 \times \pi \times r_{m} \times \frac{180^{\circ}}{360^{\circ}}=189 \mathrm{~mm} \\
r_{m}=60.2 \mathrm{~mm}
\end{gathered}
$$

To calculate the minimum torque rating of the servo motor with a maximum expected force of $20 \mathrm{~N}$ and a motor pulley radius of $\mathbf{r}_{m}$.

$$
\tau_{m}=F_{m} \times r_{m}=20 \times 0.0602=1.20 \mathrm{Nm}=12.2 \mathrm{kgcm}
$$

A Futaba S3306MG $\mathrm{MG}^{5}$ servo motor was selected based on $\tau_{m}$, cost, operating speed and the need for dual ball bearings and metal gears for strength. The torque of the motor was rated at $24.0 \mathrm{kgcm}$ at $6.0 \mathrm{~V}$, which exceeded

\footnotetext{
${ }^{5}$ http://www.gpdealera.com/cgi-bin/wgainf100p.pgm?I=FUTM0021\%C2\%A0
} 
the required specification. The increased torque rating of the motor allowed the motor's pulley to increase in size to provide additional linear displacement. The updated specifications and calculations are as follows: Servo motor torque: $24.0 \mathrm{kgcm}$

Servo motor speed: $0.16 \mathrm{sec} / 60^{\circ}$ with no load

Motor pulley radius $\mathrm{r}_{m}: 75 \mathrm{~mm}$

Linear displacement: $236 \mathrm{~mm}$

Approximate number of shaft rotations: 6.25

The servo motor pulley was screwed onto a 4-arm plastic servo horn that came with the motor. The servo was connected to the PC via a Phidget Servo Controller Board ${ }^{6}$.

A stepper motor was also bought to increase the flexibility of the test rig. Unlike servo motors, stepper motors do not have a limited operating angle. This allowed the pulley attachment for the stepper motor to be smaller in diameter and yet still have the flexibility to increase the number of damper's shaft rotations. Similar design calculations were made to purchase a 3307 Stepper motor ${ }^{7}$. The stepper motor had a torque rating of $12.5 \mathrm{kgcm}$ at $12.0 \mathrm{~V}$ and the stepper pulley attachment was calculated to have a radius of $20 \mathrm{~mm}$. The stepper motor was attached to the PC via the PhidgetStepper Bipolar 1-Motor controller board ${ }^{8}$. Although the stepper motor was not needed in this study, it still provided the design with flexibility which could be used in other applications.

The pulleys for both the servo and stepper motors were 3D printed using the PLA extruder. Once again additional work was required to file the pulleys to provide a smooth surface for the nylon cord.

\footnotetext{
${ }^{6} \mathrm{http}: / /$ www.phidgets.com/products.php?category11 \& 1 product_id=1061_1

${ }^{7}$ http: / / www.phidgets.com/products.php? category $=23 \&$ product $i d=3307 \_0$

${ }^{8}$ http:/ / www.phidgets.com/products.php?product_id $=1063$
} 


\subsubsection{Test rig construction}

A scaled model of the test rig was made in SolidWorks to conceptualise the size of the test rig, and to determine the spacing and placement of components. As shown in Figure 3.7, the components were fixed to a backboard to ensure they remained aligned to produce repeatable results. The backboard was made from MDF. A slot was used to allow the vertical position of pulley $\mathrm{P}_{2}$ to be adjusted so that it could accommodate dampers ranging from 55 to $140 \mathrm{~mm}$ in height.

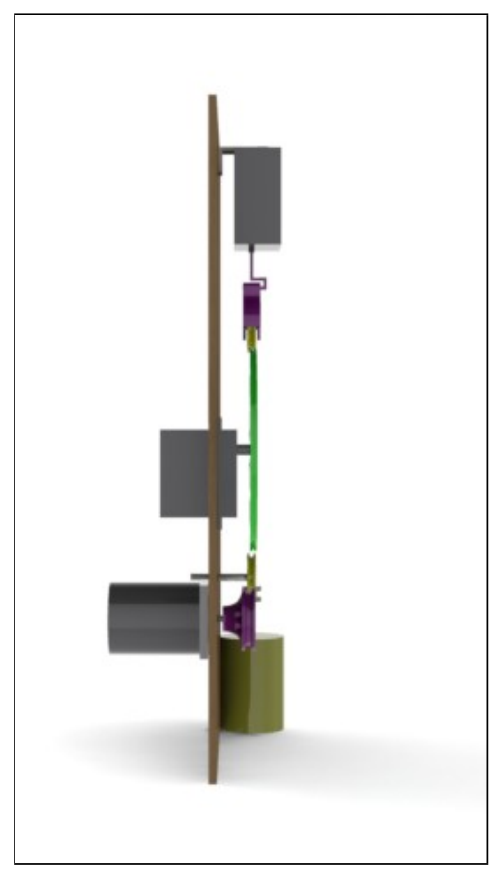

(a)

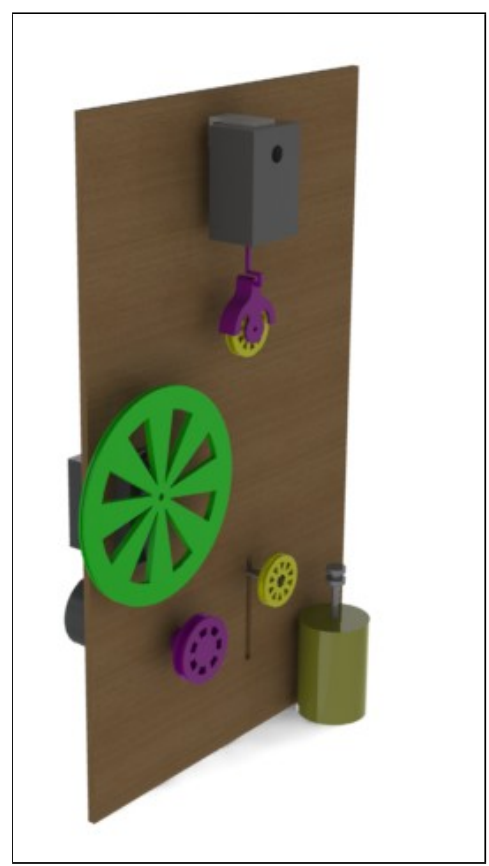

(b)

Figure 3.7: SolidWorks rendering of the MDF backboard and components of the test rig. 3.7a The components of the test rig were fixed to the backboard to ensure they were aligned. As shown, pulley $\mathrm{P}_{1}$ was attached to the force sensor, rather than to the backboard, allowing it to move vertically. 3.7b Isometric view of the test rig gives an indication of the spacing between components and shows the slot used to vary the vertical position of pulley $\mathrm{P}_{2}$. 
A saddle clamp (shown in Figure 3.8b) was used to fix the damper to the backboard to prevent the damper from moving during operation.

The framing for the test rig was made from $20 \times 20 \times 3 \mathrm{~mm}$ square aluminium tubing. Aluminium was chosen as it is easy to machine, low cost and light weight, which allowed the test rig to be portable. The backboard was attached to the middle of the frame to balance the weight of the components and ensure the test rig remained steady during operation. A framed structure was chosen as it allowed easy access to the components and reduced the amount of construction materials required. SolidWorks model used to determine component position and a comparison of the constructed test rig can be seen in Figure 3.8.

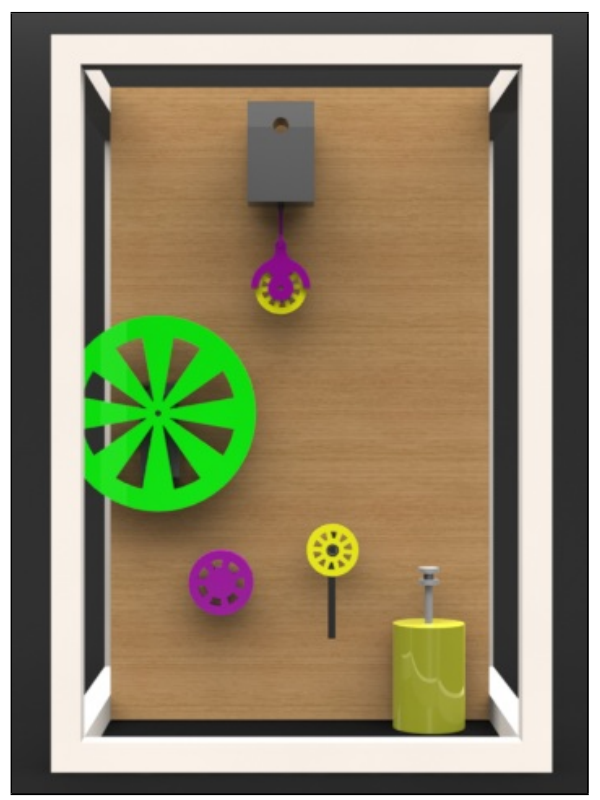

(a)

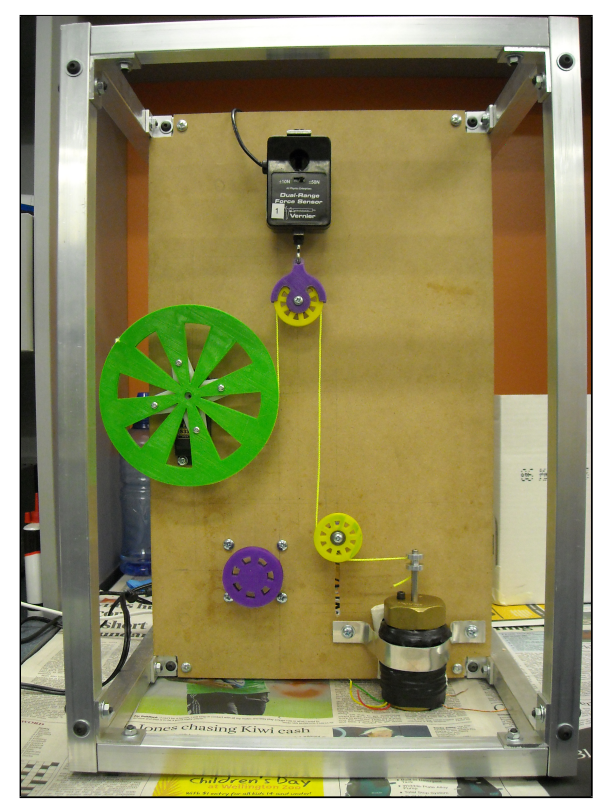

(b)

Figure 3.8: Completed pulley test rig design. 3.8a SolidWorks model of the test rig used to visualise the location of components to aid construction. $3.8 \mathrm{~b}$ Final constructed test rig showing the components attached to the backboard and frame. As shown by the cord, the components are vertically and horizontally aligned. 


\subsection{Damper Housing and Vane Designs}

Following from the previous sections the rotary damper needed to be designed and built. The damper had four components: the housing, shaft and pulley, and the mechanical interaction with the fluid through the use of vanes. The housing needed to be 'water tight' to prevent the enclosed fluid from escaping. The inside of the housing needed to be accessible to allow the three vane designs chosen to be tested. These designs were the discs, paddle and helix. To lower the cost of the damper, both the housing and the vane designs needed to be simple to construct and could not rely on high precision manufacturing.

\subsubsection{Rotary damper housing}

The housing of the damper needed to provide an enclosure for the vane to rotate within the fluid. The housing was assembled from standard plumbing fittings as they are readily available. Screw on end caps allow easy access to change the vane designs and replace the fluid as needed (Figure 3.9). Brass fittings were chosen as they are easily machined and as brass is non-magnetic it does not distort the generated magnetic field. The dimensions of the parts were limited to standard stock sizes, but the total size of the housing was still within the bounds of Objective One. Due to the machining of the brass tubing, the inner diameter was not consistent, but varied from 32 to $34 \mathrm{~mm}$, which sometimes caused problems during testing (see Section 4.3.3). As the end caps had an approximate thickness of $1.5 \mathrm{~mm}$, the scope for modifications was limited and it was not possible to use seals or bearings. Seals, although potentially useful, were not considered essential. A locater well was drilled into the bottom cap to keep the shaft vertically aligned (see Figure 3.9 right). As the test rig was designed to hold the damper upright leaking of the fluid was limited. Three holes were drilled into the top cap, a hole for the shaft to pass, one to fill the damper with fluid and the other to release air while filling. 
Preliminary tests containing water in the housing showed that water was able to leak out from between the brass tubing and the bottom cap. Thread seal tape was used to prevent this fluid leakage and tests were repeated, with additional seal tape added each time, until there were no visible signs of leaking or water level reduction in the housing.

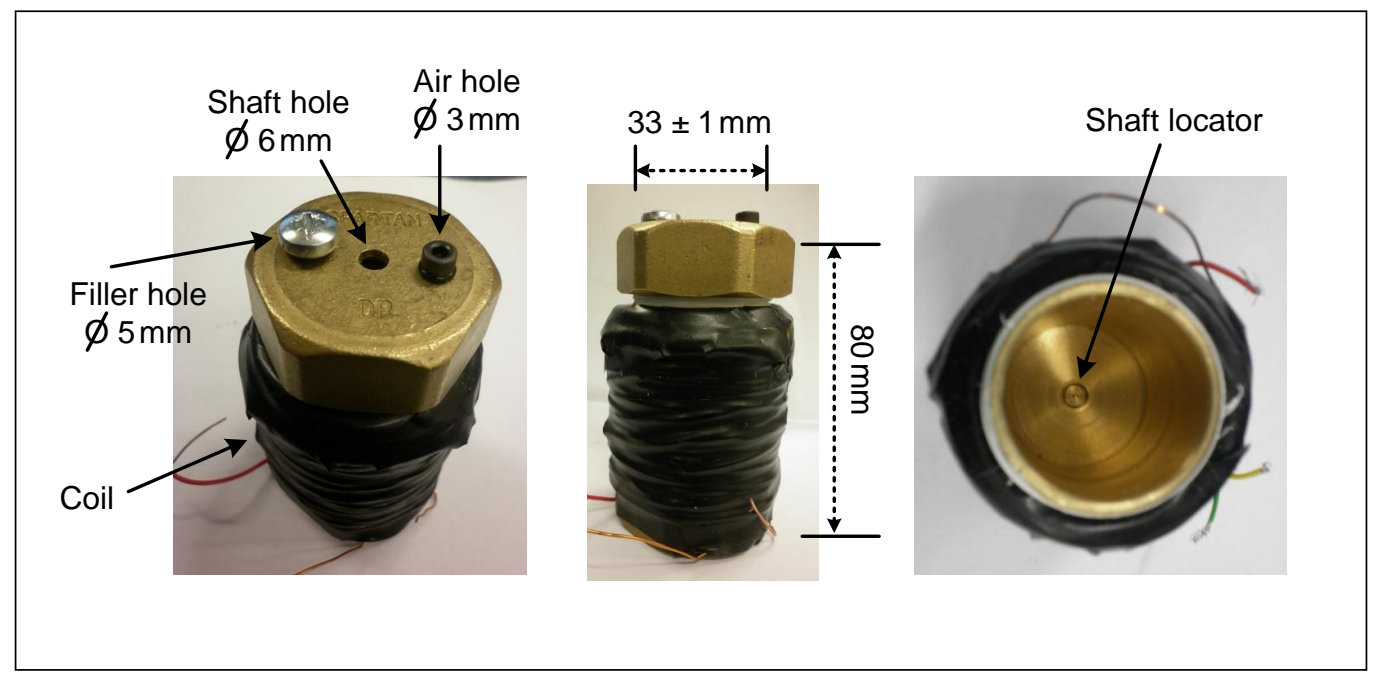

Figure 3.9: Brass housing indicating dimensions and points of interest. The length and width measurements refer to the inner dimensions of the tubing.

Copper wire with a diameter of $0.71 \mathrm{~mm}$ was used to make the electromagnet coil. Its large diameter provided a low resistance, but was flexible enough to wind the coil by hand. The damper contained approximately 370 turns. The orientation of the coil winding produced a magnetic field parallel to the shaft.

To monitor the temperature of the damper as was required in Objective One, a LM35 temperature sensor was attached to the outside of the brass tube, between the housing and the coil. The inclusion of the temperature sensor meant that the magnetic field generated was not uniform, however this would be a very small effect and have no discernible impact on the operation of the damper. 


\subsubsection{Rotary vane designs}

In addition to the commonly used disc design for rotary dampers, two alternative vanes designs, a paddle and a helix, were investigated. The designs were based on how their expected interaction with MR fluid could create a large output torque range and ease of manufacture. The following section describes the vane designs, how they were expected to interact with the fluid and their construction. The construction of the vane designs had a loose tolerance of approximately $1 \mathrm{~mm}$, this was realistic as the damper was required to be low cost and easy to manufacture.

\section{Discs design}

Rotating discs were included in the research as they are the most commonly known and used design for rotary dampers (Figure 3.10). As the disc rotates its shears the iron chains within the fluid, increasing the torque required to turn it. As the discs only shear the fluid, the damper has the disadvantage of not quickly (within a few rotations) dispersing iron particles that have settled.

As discussed in Section 2.3.2, increasing the surface area of the disc and reducing the size of the fluid gaps increases the output torque. As the width of the housing was limited by the overall size of the system, multiple discs were stacked to increase the surface area. Achievable manufacturing tolerances limited the size of the fluid gap.

Due to the complexity of the design and the required machining time, the design was 3D printed in PLA $^{9}$ (Polylatic Acid). Therefore the construction of the pieces were limited by the accuracy of the printer and the material properties of PLA. The minimum practical thickness of the discs was $3 \mathrm{~mm}$ as thinner discs resulted in bent and weak pieces. In total there were seven rotating discs with square slots (for the square shaft),

\footnotetext{
${ }^{9}$ http://www.matbase.com/material/polymers/agrobased/polylactic-acid$\mathrm{pla} /$ properties
} 
each of which was positioned between two stationary discs. The gap between each moving and stationary disc was $2 \mathrm{~mm}$. Washers were printed and used to hold the rotating discs equidistant between stationary discs. The stationary discs and an outer casing were printed as one piece and attached to the brass housing with silicone sealant. A square aluminium shaft was used to rotate the discs. As noted in section 4.1.1, due to the $0.4 \mathrm{~mm}$ accuracy of the printer, the discs were not perfectly circular and the layered printing resulted in coarse pieces that needed to be filed to fit together. It was unknown what effect the rough surface would have on the shearing of the fluid. One disadvantage of the multiple disc design was that the many required parts would increase manufacturing cost and assembly time of the damper.

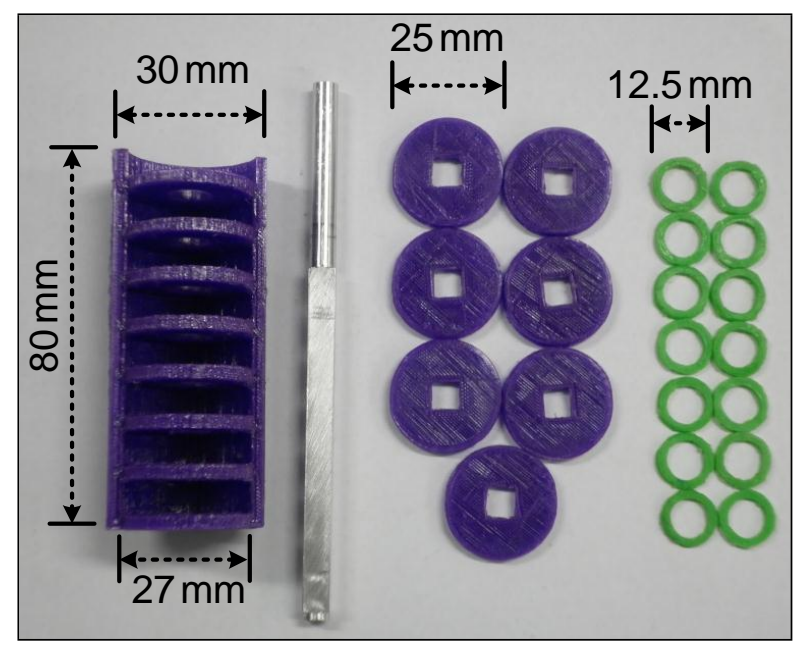

(a) Required parts

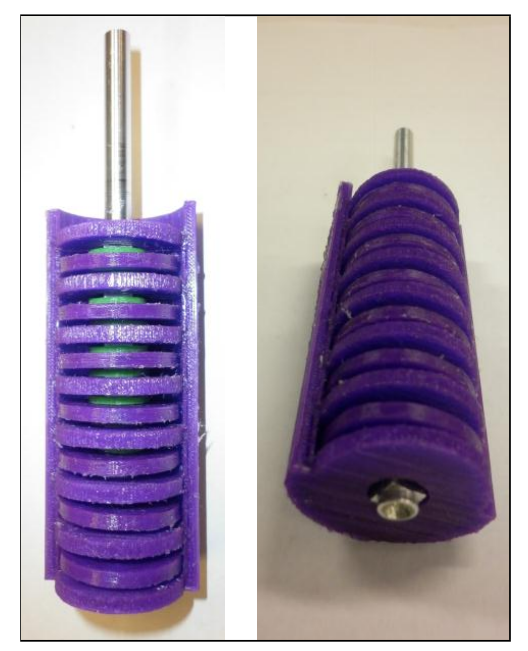

(b) Assembled view

Figure 3.10: Rotary disc design. 3.10a displays the required parts and dimensions. $3.10 \mathrm{~b}$ Assembled disc design, note that the shaft is circular at the bottom to fit inside the locater well in the bottom cap. 


\section{Flat paddle design}

An alternative to the disc design was the flat paddle vane shown in Figure 3.11. The simplicity of the paddle allowed it to be easily machined and aluminium was used as it is non-magnetic.

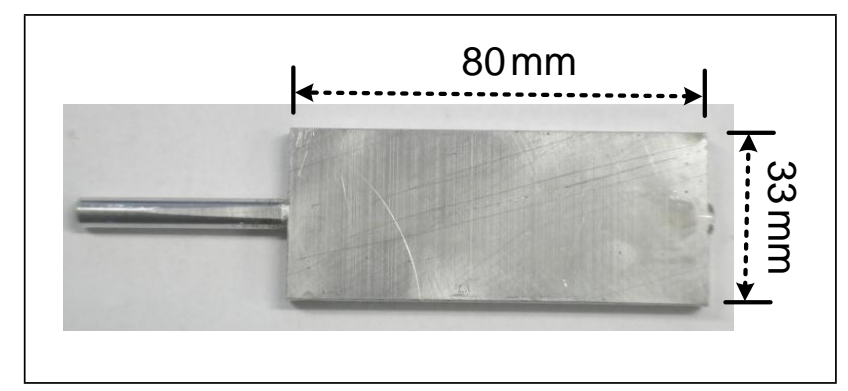

Figure 3.11: Flat paddle vane design with dimensions.

There were two hypotheses as to how the paddle interacted with the MR fluid. The first explanation was that as the paddle rotates it slices through the fluid breaking the iron chain structures. As the magnetic field strength is increased, it becomes harder for the paddle to break the chains, increasing the viscous torque. The second hypothesis is that the major cause of the change in viscous torque is due to the paddle moving the fluid as a bulk when it rotates. As the magnetic field strength is increased, the fluid behaves more like a solid, resisting the rotation of the paddle. In the "bulk" model the resistance to motion would come from wall interactions.

Determining which of the hypotheses was correct was beyond the scope of this research, as it was the relation between the strength of the applied magnetic field and the viscous torque that was to be investigated. Nonetheless preliminary experiments to understand the interaction were conducted (see Section 4.4.4). In either case an increase in magnetic field strength would increase the output torque and the interaction between the paddle and fluid during operation would help keep the iron particles suspended. 


\section{Helix design}

The final vane design was a helix, which was considered a combination of the disc and paddle designs (Figure 3.12). Similar to the disc design, as the vane rotates it shears the fluid. Increasing the magnetic field strength increases the fluid's resistance to shearing. The angled path traveled by the helix causes the vane to slice through the fluid, breaking the chain structures. This was assumed to be a similar mechanism to the paddle design's operation. The movement of the helix vane was also assumed to help disperse any settled iron particles.

The helix vane was 3D printed with a support structure, as the complicated design would have been time consuming to machine. The support structure was removed and filing was needed to allow the vane to rotate smoothly inside the housing. An adhesive was used to fix a stainless steel shaft through the vane. Unfortunately, the adhesive dried before the shaft was able to be pushed all the way through to allow an extra $1 \mathrm{~mm}$ to sit inside the locater. Therefore the helix vane sat flush on the bottom cap.

As the operation of the helix is analogous to an Archimede's screw, some amount of pumping was expected. This could result in the production of high pressure regions within in the fluid which would oppose the vane rotation. The severity of any pumping effects needed to be investigated (see Section 4.1.3). 


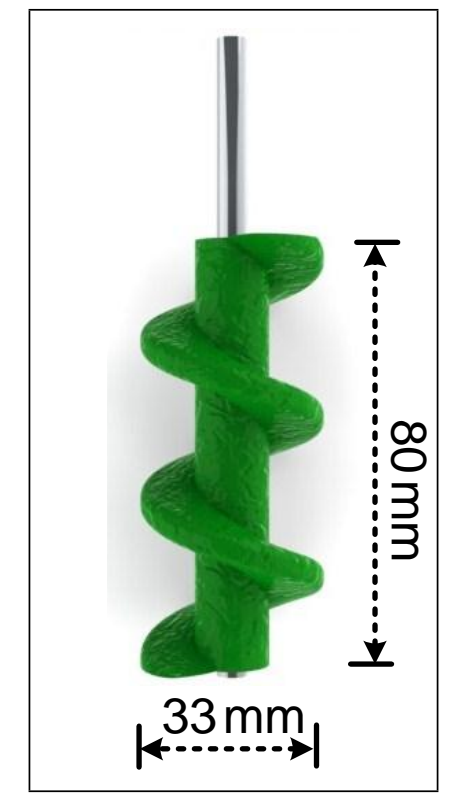

(a)

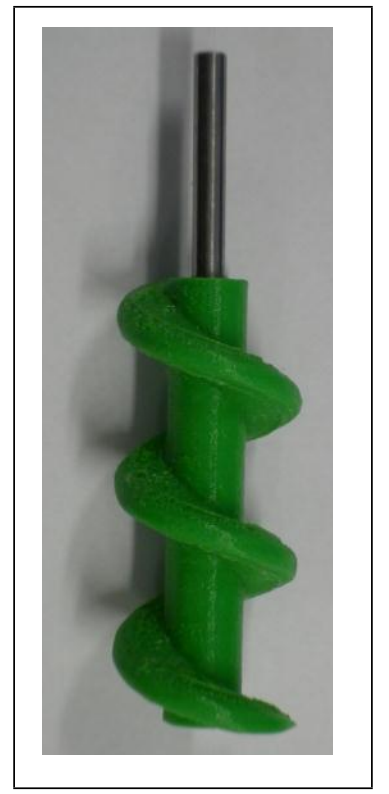

(b)

Figure 3.12: Helix vane design with dimensions. 3.12a SolidWorks rendered helix and printed helix $3.12 \mathrm{~b}$ noting the similarities

\subsection{Test Rig Operation}

The test rig was required to quantify the performance of each of the three vane designs and monitor the system's operating temperature. This was achieved by measuring the opposing force generated by each design and the temperature of the damper's coil. The opposing force was measured for varying values of the damper current and the motor's rotational speed.

A LabVIEW ${ }^{10}$ program was created to operate the test rig by controlling the servo motor and collecting measurements. LabVIEW was chosen as it was able to interface with the Vernier LabPro, National Instruments DAQ and the motor controller boards. The code can be found in Section A and the block diagram of the hardware setup is shown in Figure 3.13. As

\footnotetext{
${ }^{10}$ http:/ / www.ni.com/labview /
} 
required in Objective Three, the program was used to sample and collect force and temperature data, which were dynamically displayed on screen during test cycles. The sample rate for the temperature was set to $1 \mathrm{kHz}$ and as the temperature measurements did not vary greatly with time, the collected measurements were averaged twice a second. The Vernier LabPro was sampled at its maximum sample rate of $40 \mathrm{~Hz}$, which was adequate for experimentation (refer to Section 3.5.1).

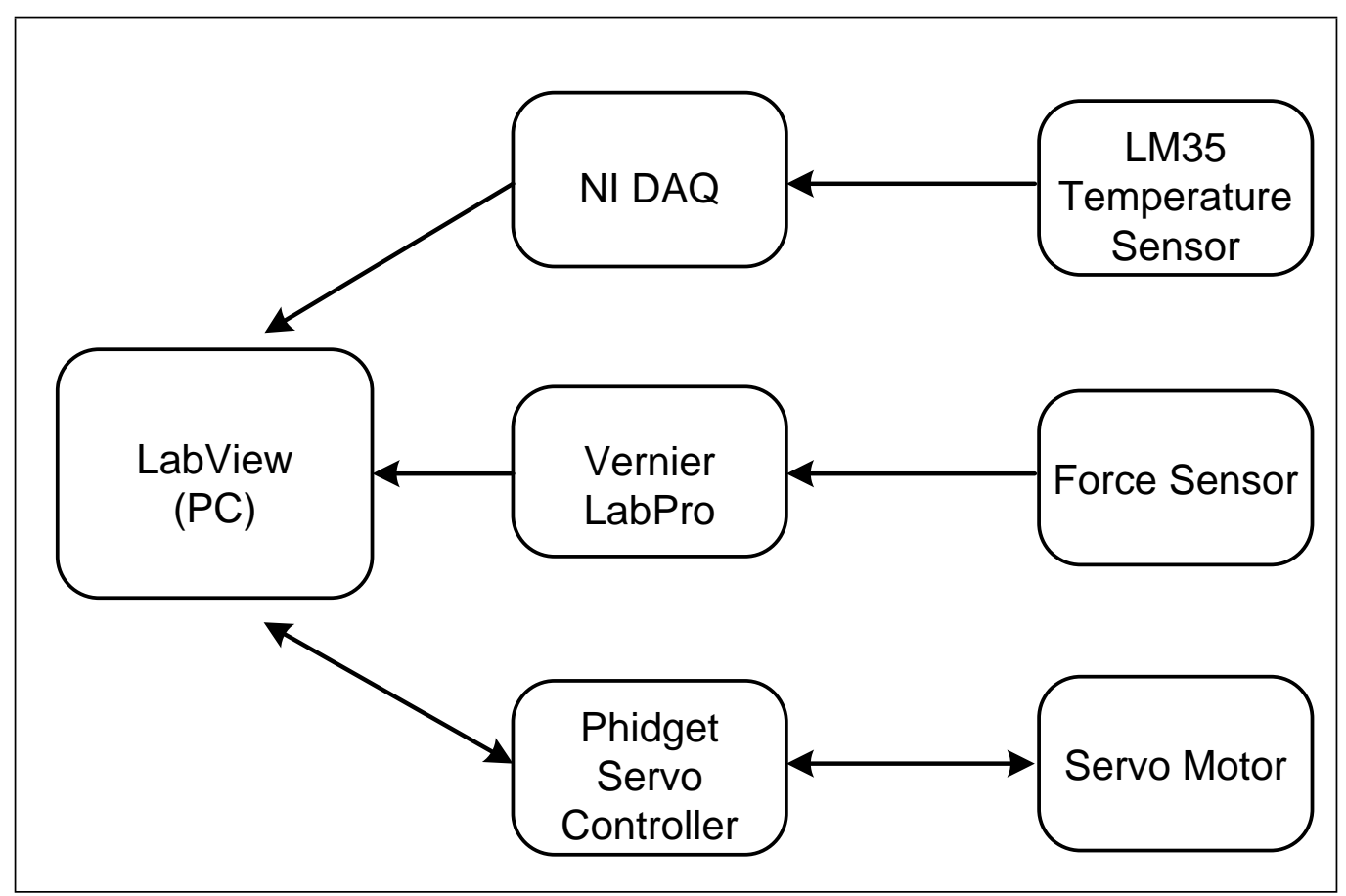

Figure 3.13: Block diagram showing how the different hardware components of the test rig interfaced with each other.

The front panel of the LabVIEW program used to control the test rig and record measurements is shown in Figure 3.14 with important features highlighted. The block diagram of the program is found in Section A. 


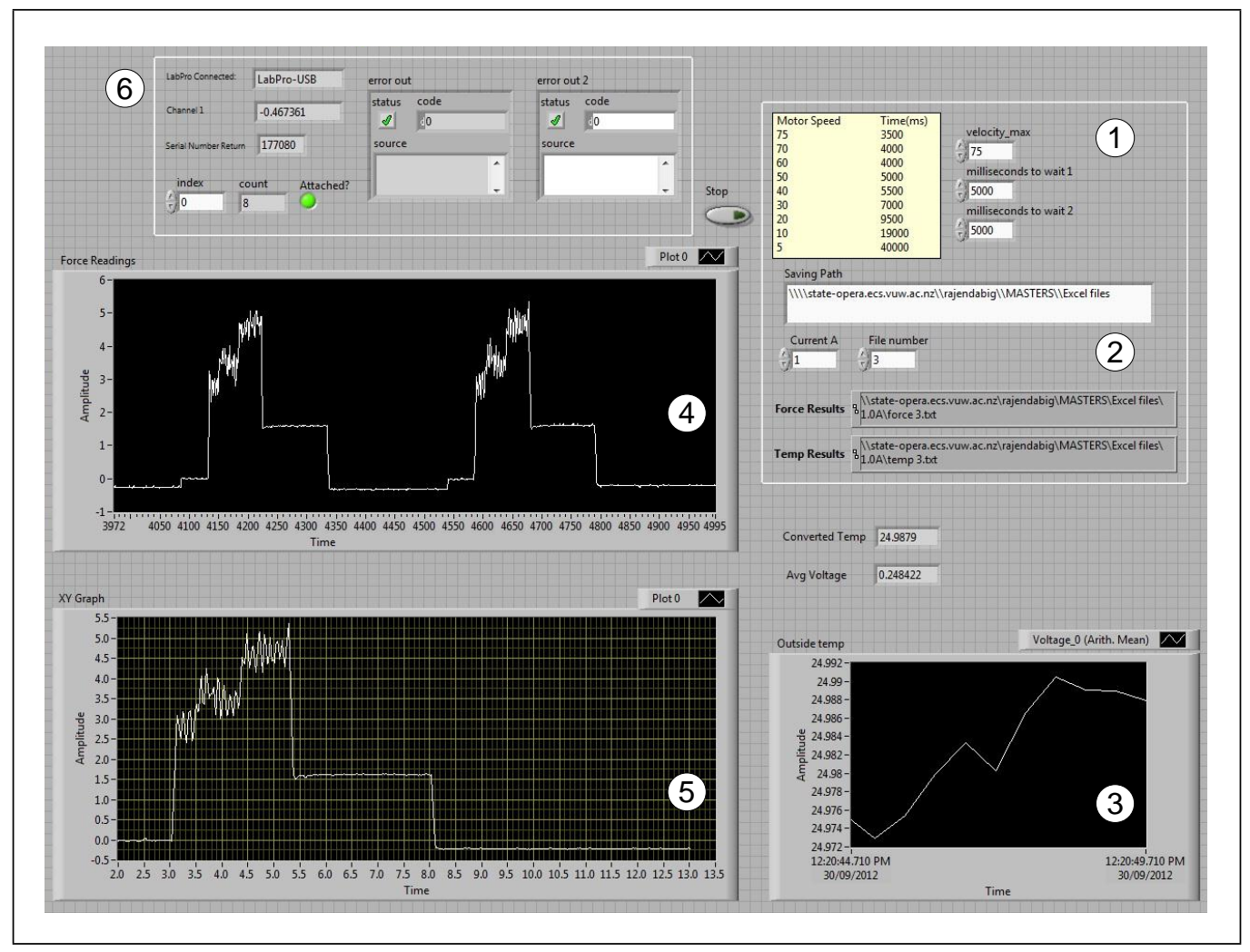

Figure 3.14: A labeled front panel of the LabVIEW program used to control the test rig and record force and temperature measurements. (1) Motor speed settings. (2) Data file specification for force and temperature measurements. (3) and (4) Dynamic displays of temperature and force measurements. (5) Force measurements for completed test cycle. (6) Notification of system errors.

With reference to Figure 3.14, the following is a description of the operation of the program and test rig:

Before the program was started, the required speed of the motor (1), and the file location of the force and temperature data (2) needed to be specified. Once the program had started, temperature measurements were logged and displayed on screen, allowing changes in temperature to be monitored (3). The DAQ had a resolution of $0.305 \mathrm{mV}$, but the accuracy of 
the temperature measurements were limited by the LM35 sensor to $2.5 \mathrm{mV}$, corresponding to $0.25^{\circ} \mathrm{C}$.

Contemporary to the logging of temperature, there was a two second wait period at the start of each test cycle. The length of this delay was arbitrary but it allowed time for the program to measure the current force value and use this value to offset all following force measurements, essentially zeroing the sensor. This calibration accounted for the weight of the pulley attachment and any drift in the force sensor between test cycles. After the calibration period, the program logged and dynamically displayed the force measurements to allow characteristics of the damper design to be identified(4).

Once the program had started logging the force measurements, there was a further arbitrary wait period of one second before the motor began rotating. The one second wait period was used to identify the beginning of the damper's rotation in the recorded data files, which was needed for post data analysis. Through the pulley system, the rotation of the motor caused the damper to be rotated. After the motor had reached its predefined end point, it held that position (the duration was dependent on the speed of the motor) before returning to its start position.

At the end of a test cycle the force and temperature measurements were recorded to text files for later analysis. The force measurements of the completed test cycle and notification of any errors that occurred were displayed. Between each test cycle the damper's pulley was rewound by hand, beginning at the same starting position for each run.

\subsubsection{Selection of motor speeds}

The range of motor speeds chosen needed to test the damper designs in similar operating conditions to that of a rehabilitation stroke device. The motor speeds were bounded by user interaction. The minimum speed of the motor was chosen to be $10^{\circ}$ / s as from the user's perspective this would 
be considered a slow but usable speed. Although a rotary damper was used, linear speed is often more intuitive to the end user. To convert the rotational speed of the motor to linear speed, Equation 3.1 is used, where $\mathrm{r}_{m}$ is the radius of the motor's pulley.

$$
\frac{2 \pi r_{m}}{360^{\circ}} \times \frac{\text { degrees }}{\text { seconds }}=\mathrm{mm} / \mathrm{s}\left(\text { where } r_{m}=75 \mathrm{~mm}\right)
$$

Considering the application of the stroke device, the range of speed of the user's hand movement would be low, approximately 0 to $80 \mathrm{~mm} / \mathrm{s}$ which from Equation 3.1 correspond to motor speeds below $75^{\circ} / \mathrm{s}$. A few faster motor speeds were chosen to investigate the changes in opposing force with velocity. For convenience the chosen motors speeds and their corresponding linear speeds are shown in Table 3.1.

Table 3.1: Selected motor speeds used and their conversion to linear speed

\begin{tabular}{|c|c|}
\hline Motor speed $\%$ s & Linear speed mm/s \\
\hline 10 & 13.1 \\
\hline 30 & 39.3 \\
\hline 50 & 65.4 \\
\hline 75 & 98.2 \\
\hline 100 & 130.1 \\
\hline 150 & 196.3 \\
\hline 200 & 261.8 \\
\hline
\end{tabular}

The maximum sample rate of the force sensor was $40 \mathrm{~Hz}$. At the maximum motor speed of $200^{\circ} / \mathrm{s}$ this equated to a measurement every $6.5 \mathrm{~mm}$ of the cord and at $10^{\circ} / \mathrm{s}$ it was $0.33 \mathrm{~mm}$, which was sufficient for this application. 


\subsection{Data Analysis Procedure}

It is important for the damper to operate consistently to allow the user and rehabilitation therapist to accurately assess and monitor the user's recovery progress. To help determine the consistency in the performance of the rotary damper designs, the force data was converted to torque and analysed statistically. This was to find variations in the measurements both within and between trials.

\subsubsection{Characteristics of force measurements}

The opposing force of the damper designs were measured to quantify the performance of each vane design. To understand the force results, the following representative force time response graph (Figure 3.15) highlights the commonly observed characteristics of the measurements and these characteristics are further discussed. 


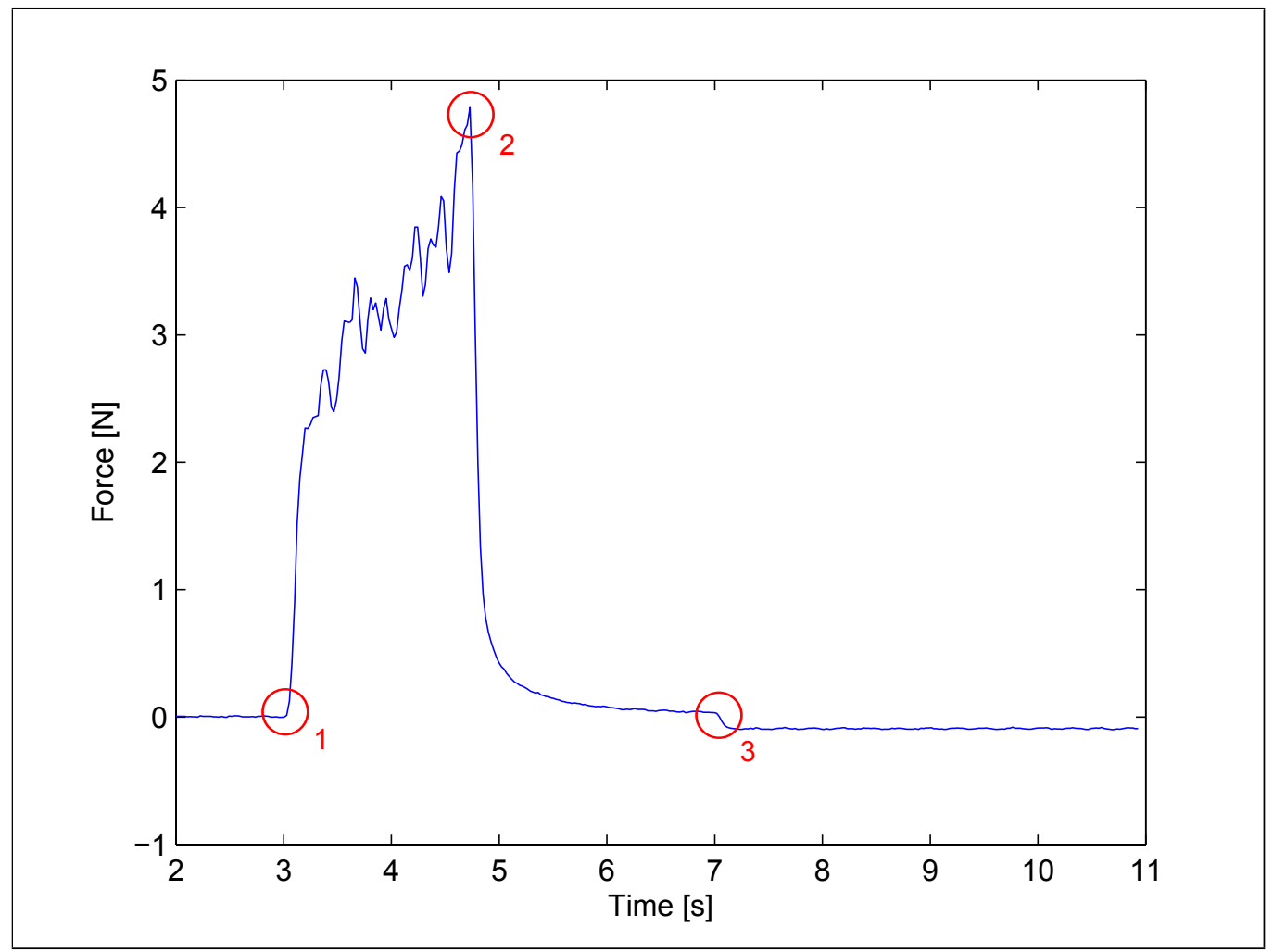

Figure 3.15: Representative force-time response measurement, noting characteristics common to all measurements. As labeled, (1) indicates the start of the motor and damper's rotation, (2) the motor has stopped rotating, ending the rotation of the damper and at (3) the motor begins returning to its start position.

The servo motor starts to rotate at point 1 (indicated by a circle in Figure 3.15), which also causes the damper to begin rotating. At point 2 the motor has reached its end position and stops rotating the damper. The motor holds its end position for a specified duration (dependent on the motor speed selected). The period between when the motor has started and when it has reached its end position, is of most interest, because during this period is when the damper is operational.

The increasing slope of the force measurements, observed when the 
damper was operating, was due to the decrease in radius of the cord wrapped around the damper's pulley. Unwinding the cord decreases the radius, which reduces the lever arm. This requires more force to rotate the damper's shaft.

Due to the abrupt stop of the motor, a spike in the force often occurred at the end of the damper's operational period. This spike and other unintentional forces caused by the servo motor distorting the force measurements needed to be accounted for when analysing the results.

After the motor had reached its end position the tension in the cord could decrease, decreasing the measured force. This occurred when the damper had enough momentum to continue rotating. As the motor moves back to its starting position, the resulting slack in the cord causes an additional drop in the force measurement.

Occasionally there were differences in the magnitude of the forces measured before the motor begins to rotate (before point 1 ) and as it returns to its starting position (after point 3). As the program used the first set of force measurements to zero the force sensor, the observed difference was dependent on the amount of slack in the cord at the beginning of the test cycle. If there was too much tension in the cord at the start, negative force readings would occur at the end, therefore the measured force was actually offset by a negative amount. However, too much slack in the cord would cause an inconsistent time delay in the collection of force data, as the motor would be pulling a slack cord at the beginning. It was important that the cord was neither too slack nor too tight. Therefore to ensure consistency between each trial, the pulley was rewound to the same starting position for each test cycle.

The pulley was unwound a total linear distance of $230 \mathrm{~mm}$ which corresponded to 6.5 rotations of the shaft. This distance would be sufficient to have the damper continuously operational during the opening and closing of a user's hand. When considering force time graphs, as the linear displacement of the test cycle was fixed at $230 \mathrm{~mm}$, the duration of each 
experiment was dependent on the set motor speed. Therefore when multiple graphs with varying motor speeds are plotted on the same figure, there is a noticeable difference in the duration of the results.

\subsubsection{Sample size}

To investigate the consistency of the damper's operation a number of trials were conducted for each motor speed and current setting, such that a statistical measure of variation could be applied. The Lilliefors test was used, which is a 2-sided goodness-of-fit test that is able to test the nullhypothesis that the data is normally distributed at a given confidence level. A $95 \%$ confidence level was used. The Lilliefors test was used instead of the Kolmogorov-Smirnov test as the parameters of the nulldistribution was unknown and needed to be estimated. The Lilliefors test was used on a sample of 30 trials, in the damper's operational region and found that the distribution of force measurements was Gaussian (Figure 3.16) within the $95 \%$ confidence level. As it was time consuming to run 30 test cycles for every motor speed and current setting, the number of trials was decreased to 10 . To check if 10 trials would still be representative of the larger set of 30 trials, the Lilliefors test was again used and within the $95 \%$ confidence level the distribution was also found to be Gaussian (Figure 3.17). 


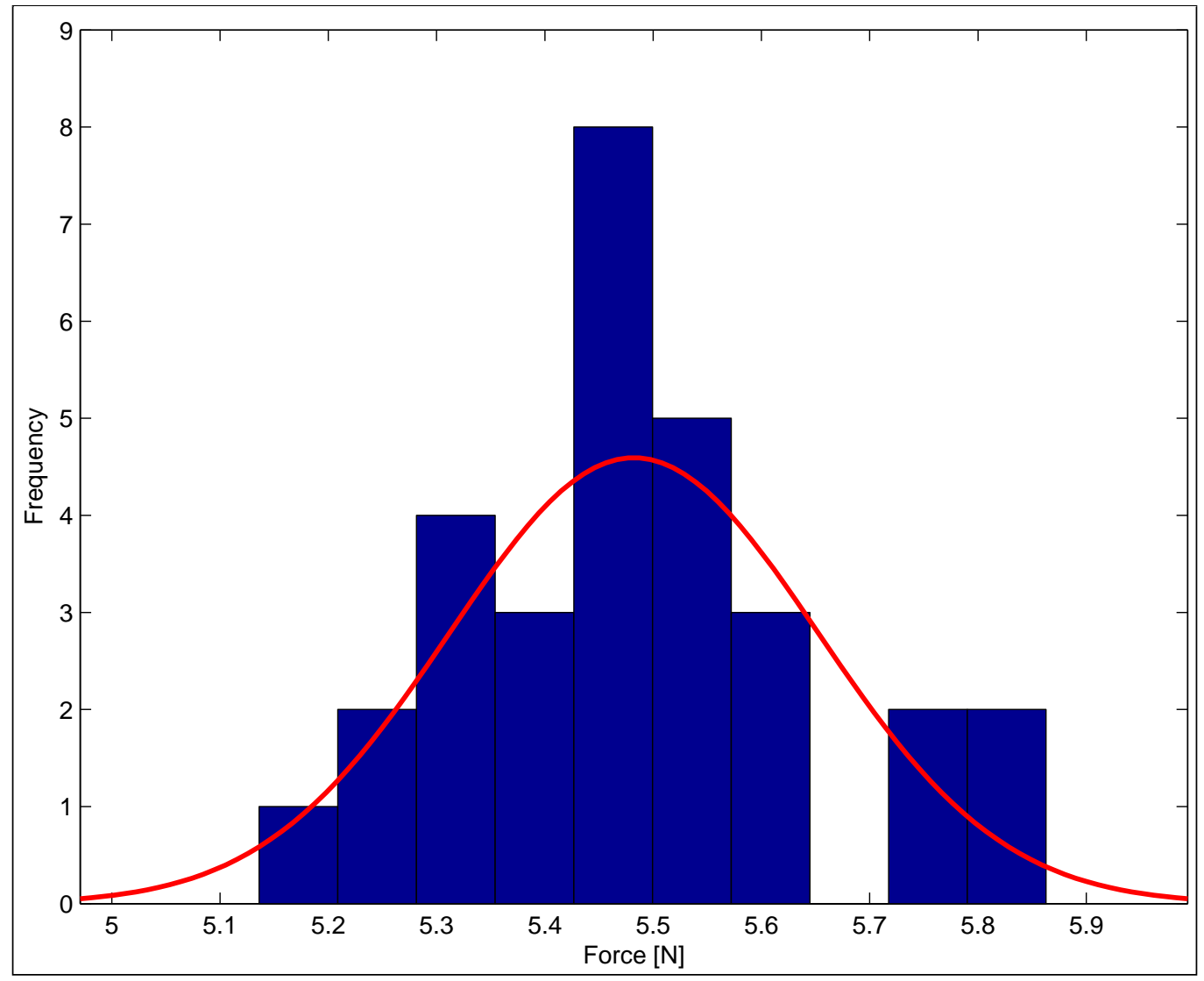

Figure 3.16: Distribution of representative sample of 30 force measurement trials, supporting the Lilliefors test that the data is normally distributed at the $95 \%$ confidence level. 


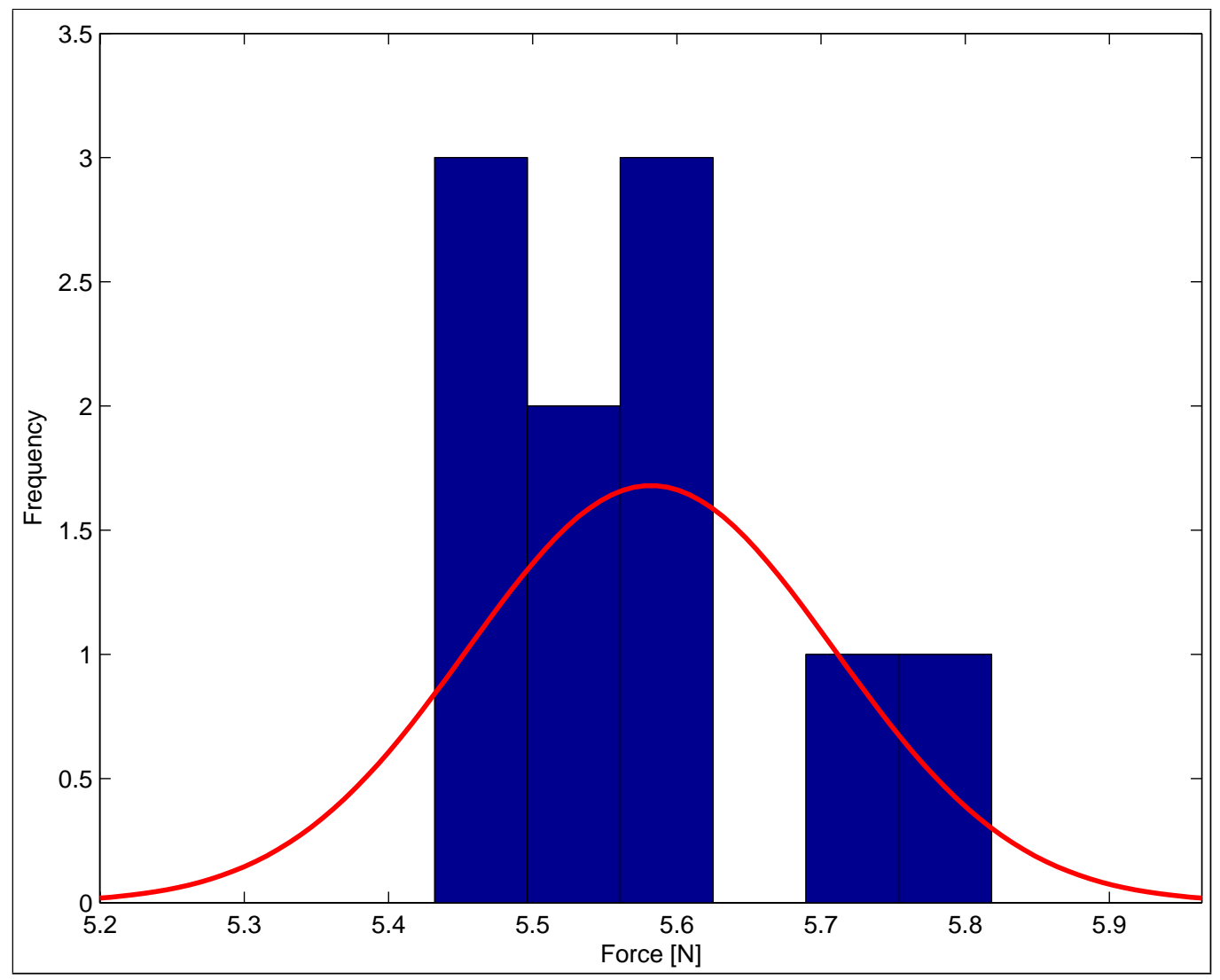

Figure 3.17: Distribution of representative subset sample of 10 force measurement trials, supporting the Lilliefors test that the data is normally distributed at the $95 \%$ confidence level.

Comparing the measured opposing force data from 30 trials (Figure 3.18) with force data using only 10 trials (Figure 3.19), the trend and range of the force measurements were similar with the exception of a couple of differences. Firstly there is a greater peak force (approximate $2 \mathrm{~N}$ higher) in the set of 30 trials, which occurs near the end of the damper's operational region. This increase in force is likely to be caused by the motor's sudden halt and would need to be compensated when the data is further analaysed. Hence it would not contribute to the analysis of the damper's behaviour. The other noticeable difference is the remaining tension in the 
cord, when the motor begins moving to its starting position in Figure 3.18 (observed by three graphs remaining at $4-5 \mathrm{~N}$ from time 4 to 6 seconds). Although this visually appears to be a significant difference between the two plots, as the remaining tension in the cord is outside the damper's operating region it would not be included in the analysis of the data. Therefore using 10 trials rather than 30 was a practicable decision.

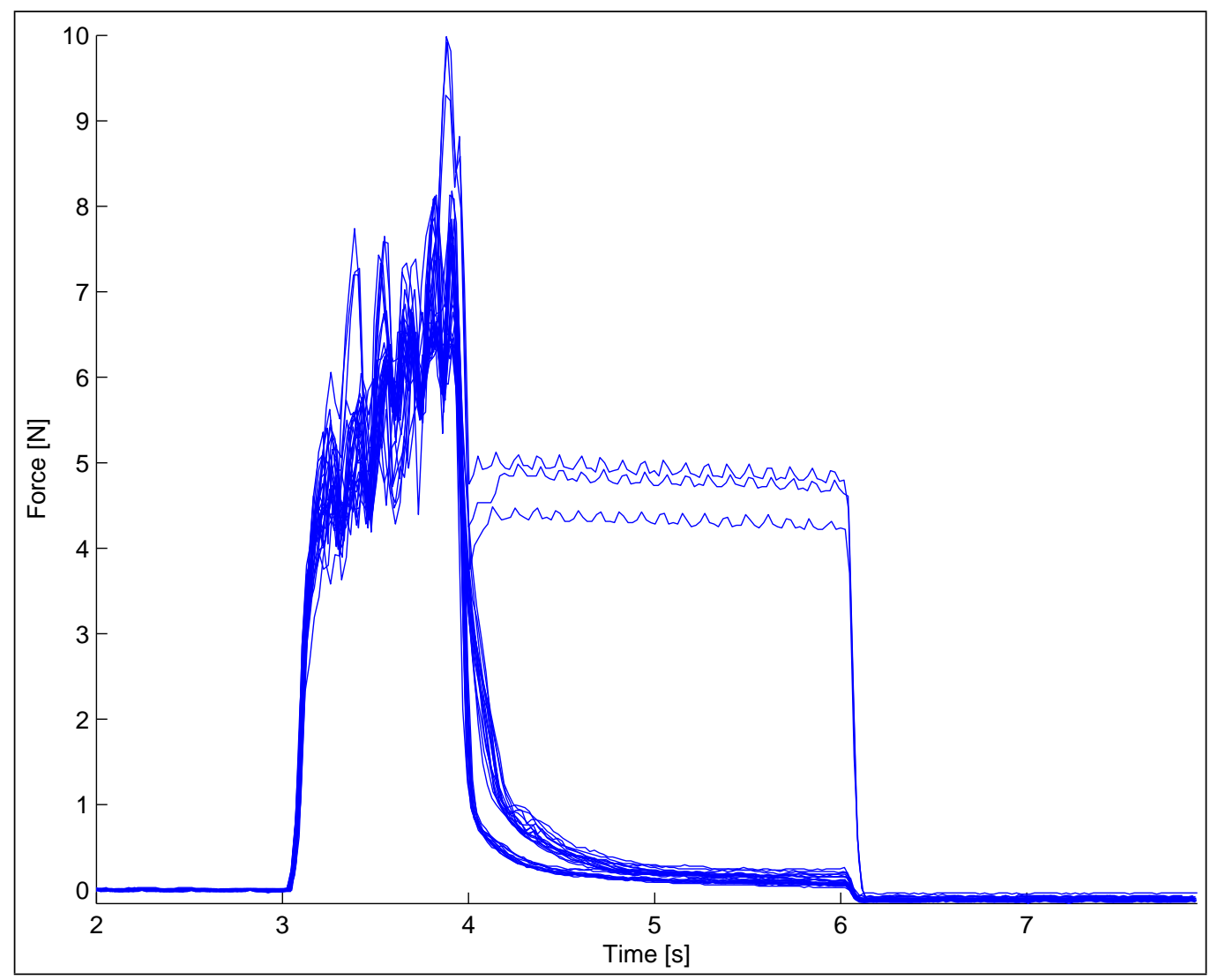

Figure 3.18: Representative sample of 30 force measurement trials. 


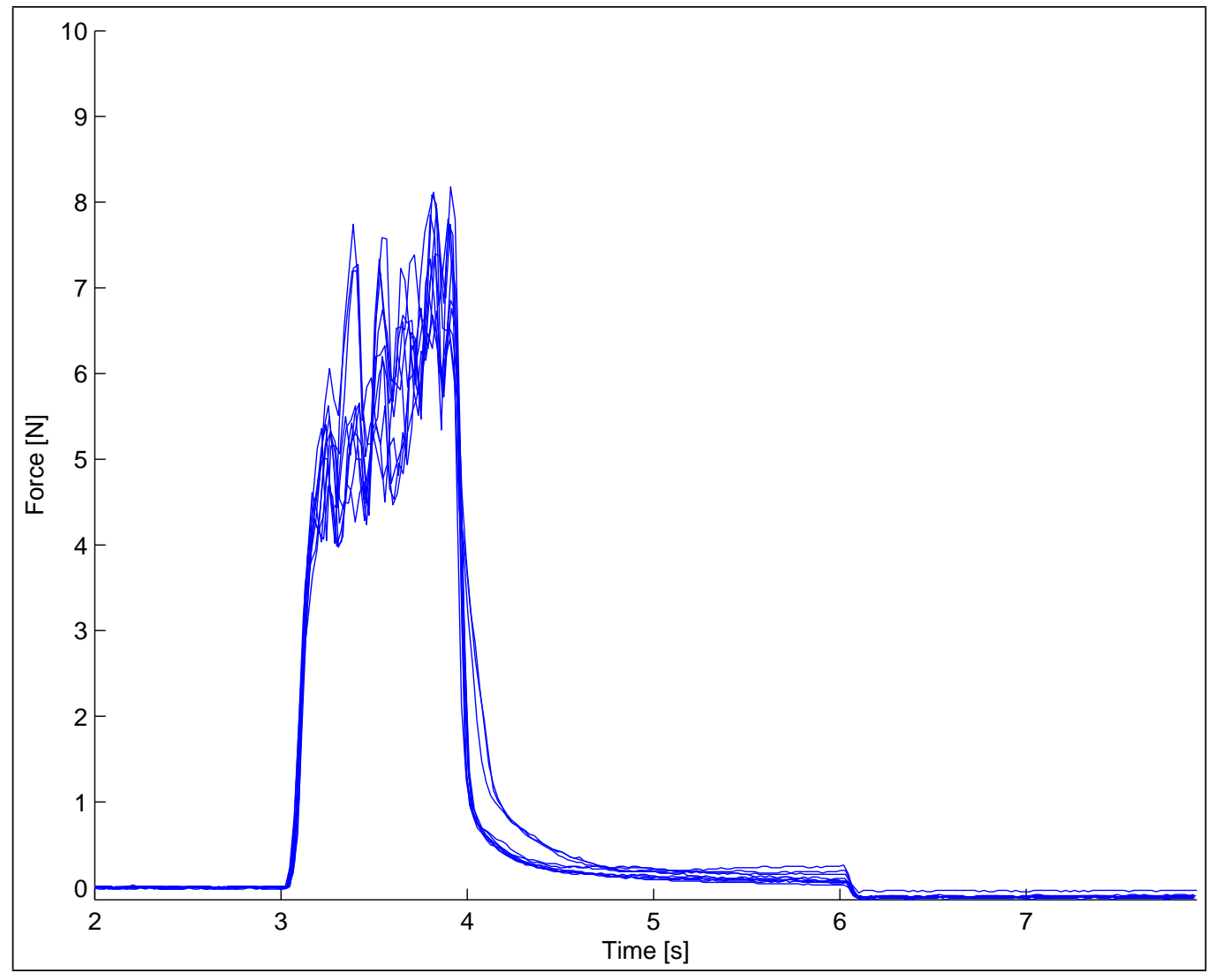

Figure 3.19: Representative sample of 10 force measurement trials, taken from the larger set of 30 trials.

\subsubsection{Averaging force graphs}

Averaged force graphs were used to visualise the damper's response with changes to the motor's speed or input current setting. The graphs were an average of the 10 trials associated with a specified setting. For a particular setting, the opposing force for 10 trials are shown in Figure 3.20 with the averaged force graph in black. 


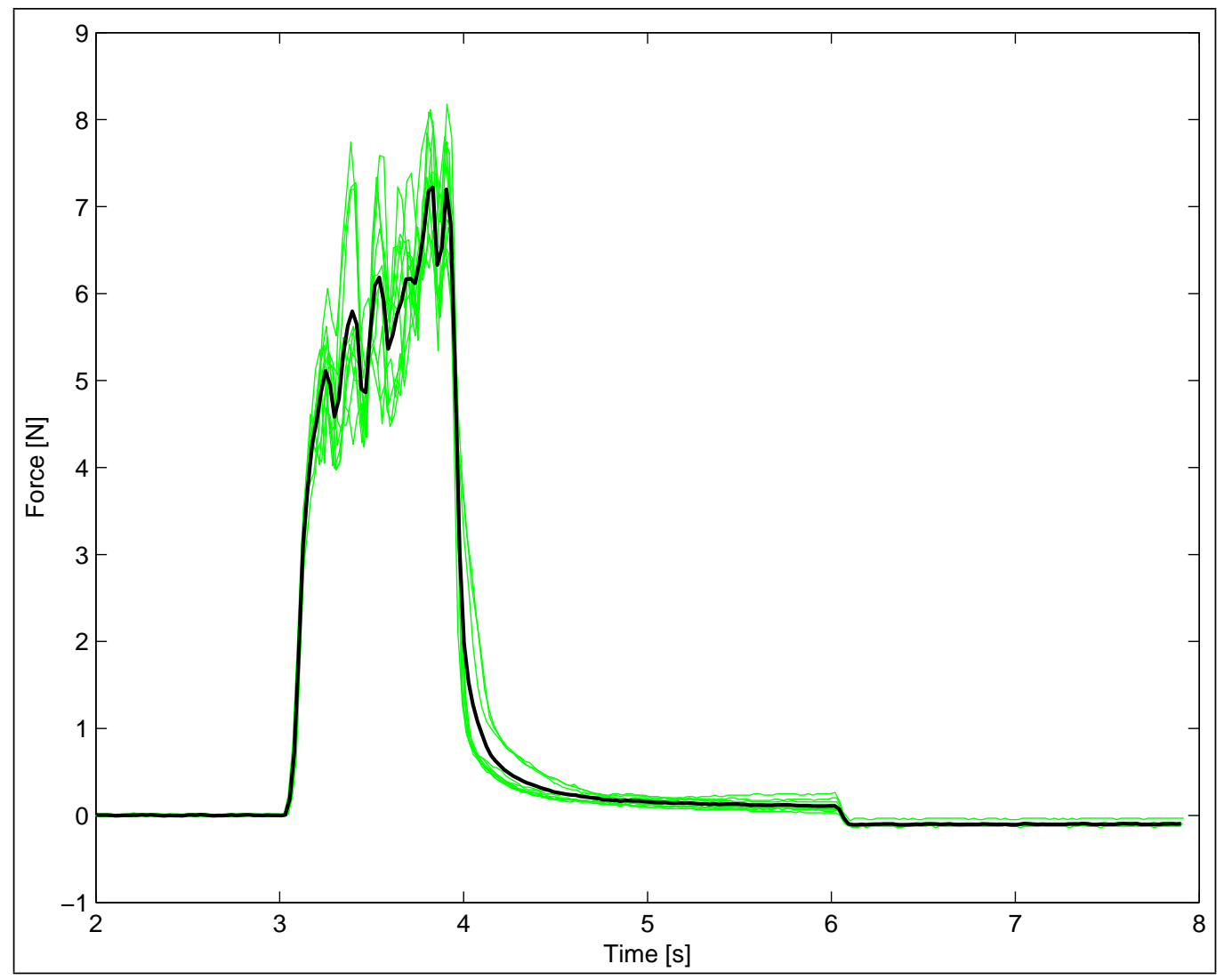

Figure 3.20: Opposing force measurements for 10 trials, at a particular speed and current setting of the damper, with the average of the 10 trials shown in black.

\subsubsection{Conversion from force measurements to torque}

As a rotary damper was used, the opposing force measurements needed to be converted to torque. As highlighted in section 3.6.1, as the damper rotated, the radius of the cord around its pulley decreased, proportionally increasing the force required to rotate the shaft. As this increase in force was not a property of the damper design it needed to be compensated when converting force to torque. The measured decrease in cord radius was from $6.5 \mathrm{~mm}$ to $4.5 \mathrm{~mm}$. 
A model of the relationship between the measured force and the generated torque in the damper was used. It would have been possible to infer the relationship between force and torque by fitting a linear trend to the force curve. However this could have masked linear trends in the torque that may have resulted from fluid effects, such as the pressurising portions of the damper, and therefore a data driven approach was not used.

Due to the alignment of the components in the test rig, the cord was considered to be pulled tangentially to the rotation of the damper's pulley.

$$
\tau=r_{p} \times F \times \sin \theta
$$

(where $\sin \theta=1$, and $r_{p}$ is the damper's pulley radius)

Using averaged measurements to compare results, could easily hide characteristics of the damper. Therefore each trial was converted individually to prevent external factors, which were not directly related to the intrinsic properties of the damper, influencing the results and subsequent findings.

To convert the force measurements to torque the data was split into three regions, shown in Figure 3.21. All force measurements prior to the start of the damper's rotation (region a) was multiplied by the maximum radius of $6.5 \mathrm{~mm}$ and all force measurements after the damper has stopped rotating (region c) was multiplied by the decreased radius of $4.5 \mathrm{~mm}$. Compensation occurred in the region where the damper operated. In this region the radius value was proportionally decreased from $6.5 \mathrm{~mm}$ to $4.5 \mathrm{~mm}$ depending on the number of force data points available. It is this compensated region, where the damper was operational that is used for further analysis. 


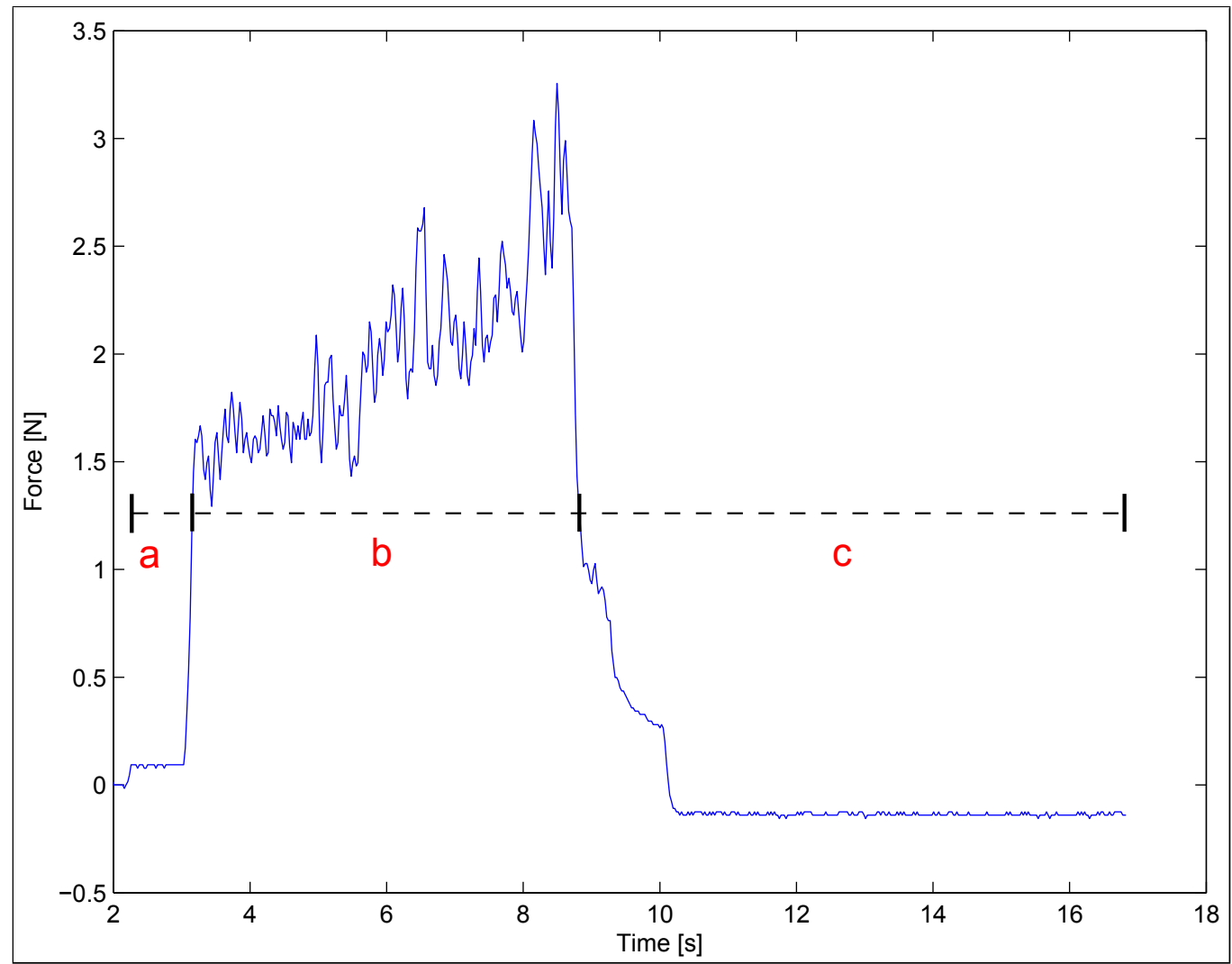

Figure 3.21: Force-time response graph showing the regions where the radius value used changes. This was to compensate for the physical change in the damper's pulley radius, to convert the force measurements to torque. (a) $r_{p}=6.5 \mathrm{~mm}$ (b) $r_{p}$ decreases from $6.5 \mathrm{~mm}$ to $4.5 \mathrm{~mm} \mathrm{(c)} r_{p}=$ $4.5 \mathrm{~mm}$

Occasionally there where times when the external factors influenced the results of the force measurements, such as force spikes caused by the halt of the motor or the damper's shaft getting stuck (see section 4.3.3). In these cases the compensated operational region of the damper was calculated as previously described, but the range of calculated torque values used was truncated as shown in Figure 3.22. 


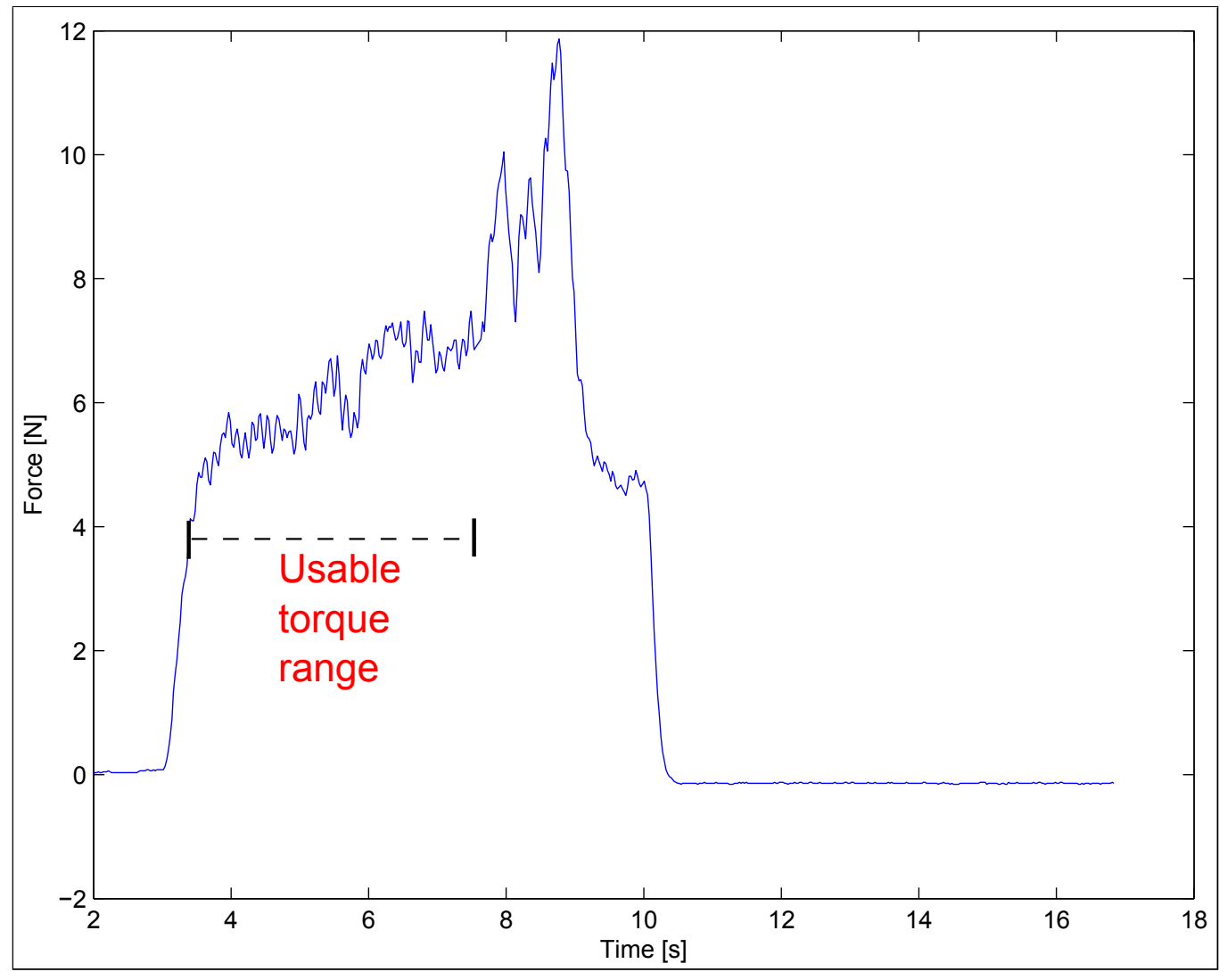

Figure 3.22: Force-time response graph showing the truncated torque measurement range used for analysis.

The difference between a compensated torque graph and an uncompensated torque graph is shown in Figure 3.23. The uncompensated graph (in red) used an average radius of $5.5 \mathrm{~mm}$ in the damper's operational region. As expected, the compensated torque graph (in black) is less steep in the operational region. 


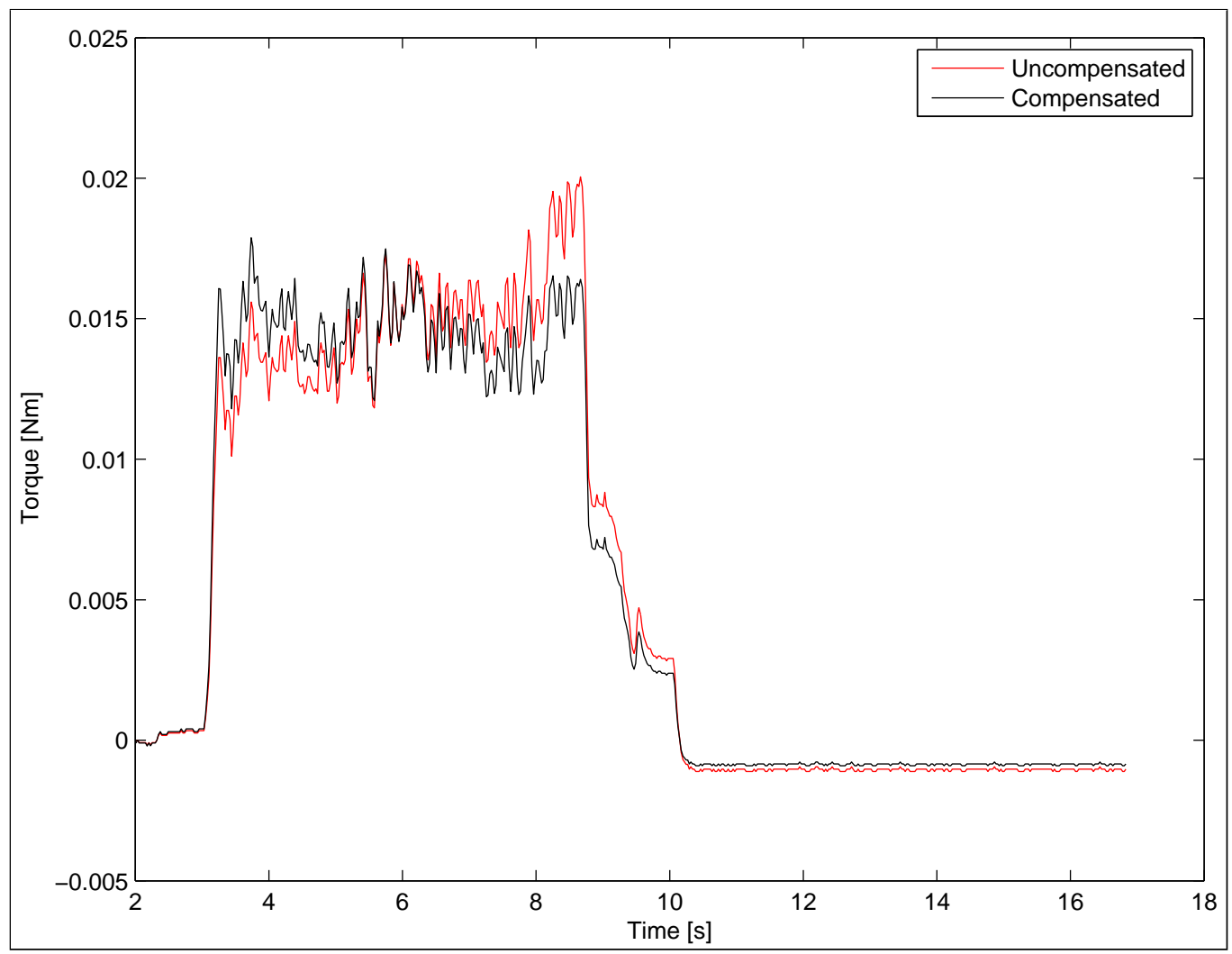

Figure 3.23: Compensated (black) and uncompensated (red) calculated torque graphs for a particular setting. The compensation in the calculation was to account for the change in radius of the damper's pulley during operation. The uncompensated graph used an averaged radius length of $5.5 \mathrm{~mm}$. Note due to the compensation in the varying radius the compensated torque graph is less steep in the damper's operational region.

\subsubsection{Torque plots statistics}

The Lilliefors test was used on a representative sample of the calculated torque data, within the damper's operational region. At the $95 \%$ confidence level the data was found to have a normal distribution. The region of the torque data used is illustrated in Figure 3.24 and the distribution of the data which supports the Lilliefors test is shown in Figure 3.25. 


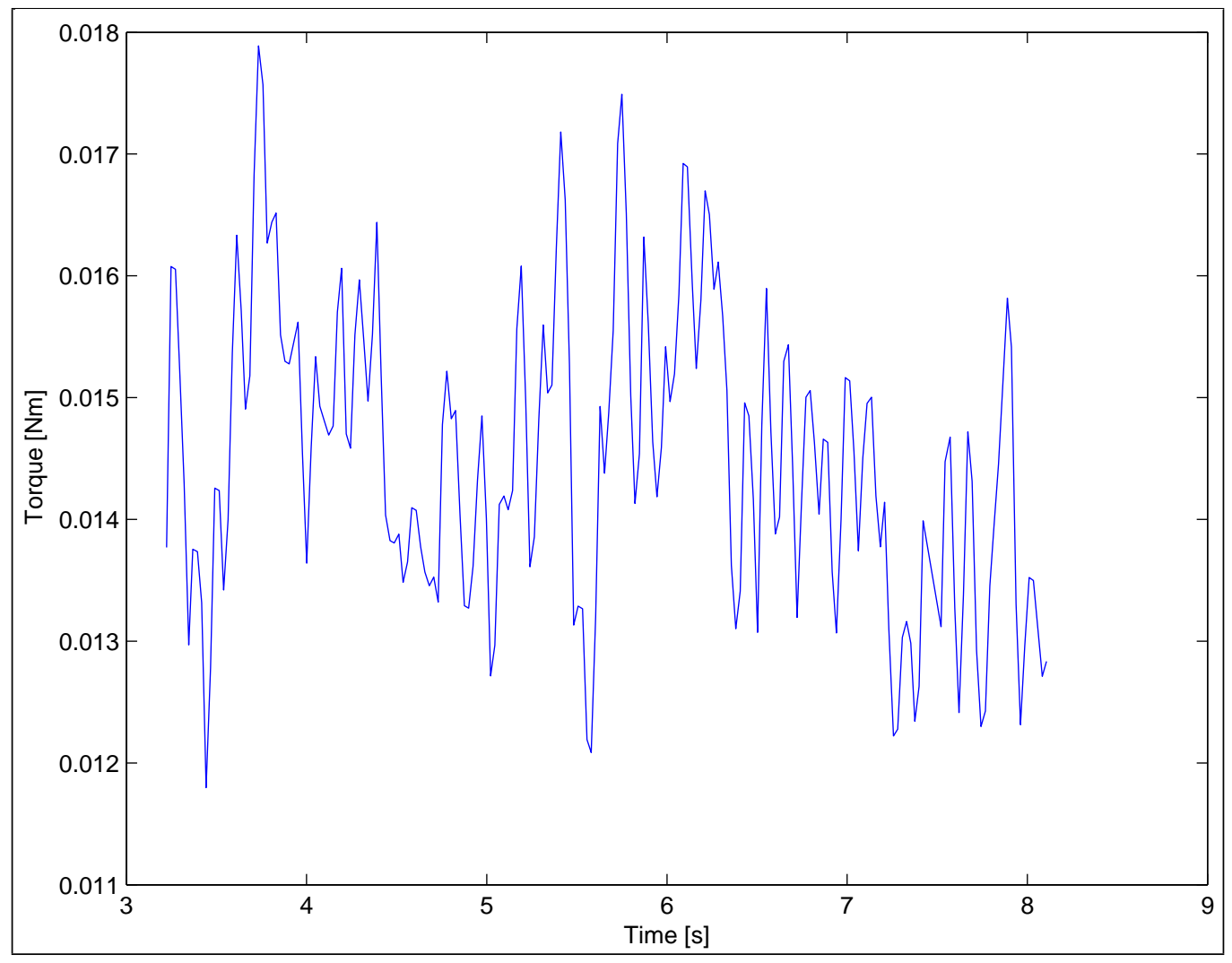

Figure 3.24: The calculated torque measurements in the operational region of the damper used to determine if the distribution was Gaussian. Note the graph is a section of one test cycle. 


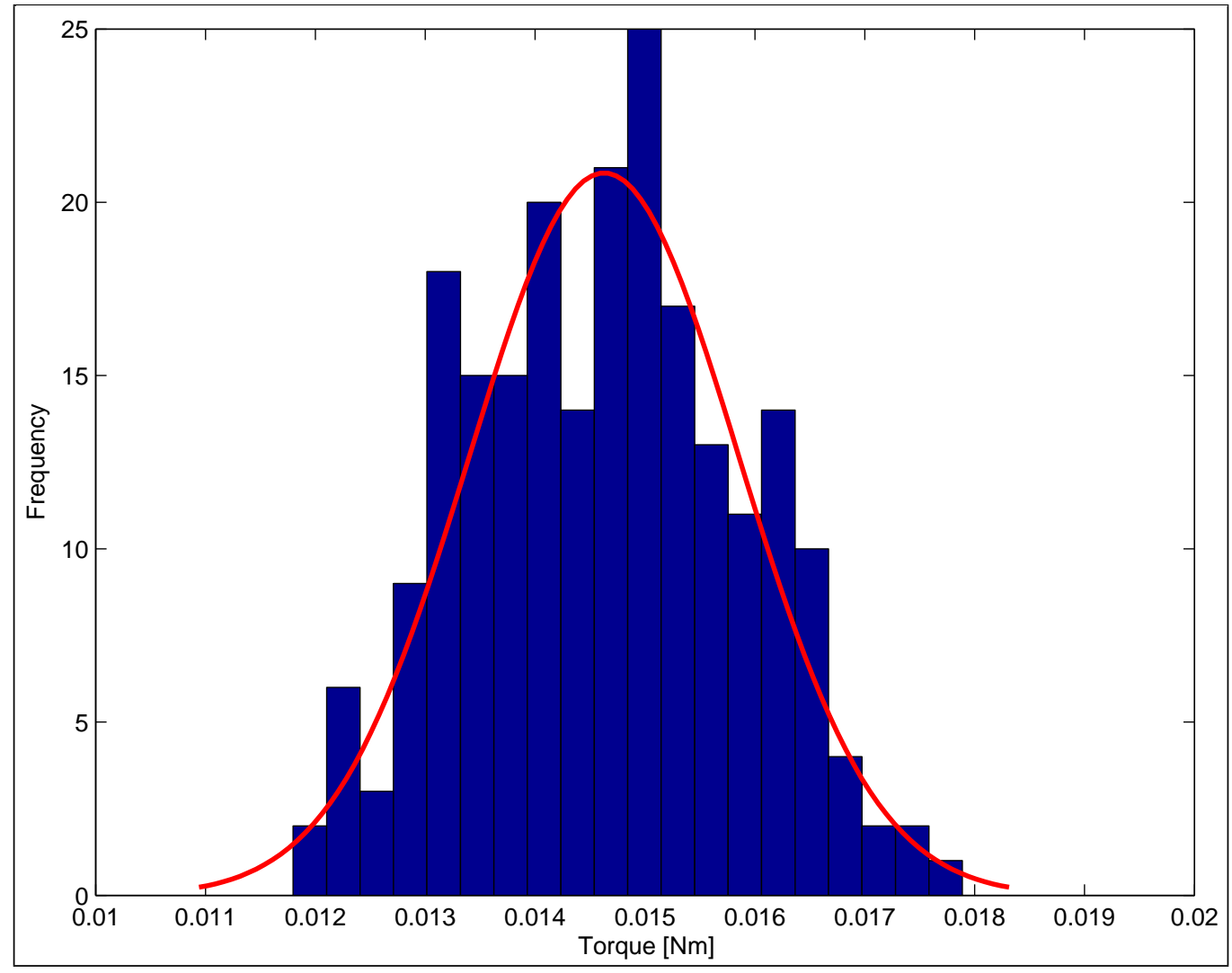

Figure 3.25: Distribution of calculated torque measurements withing the operational region of the damper. The plot supports the test that the torque results are normally distributed at the $95 \%$ confidence level.

To help determine the consistency of the performance of each damper design, the mean and standard error were calculated. These values were used to characterise the variance of measurements within a trial and the spread in the measurements over the 10 trials (Figure 3.26). 


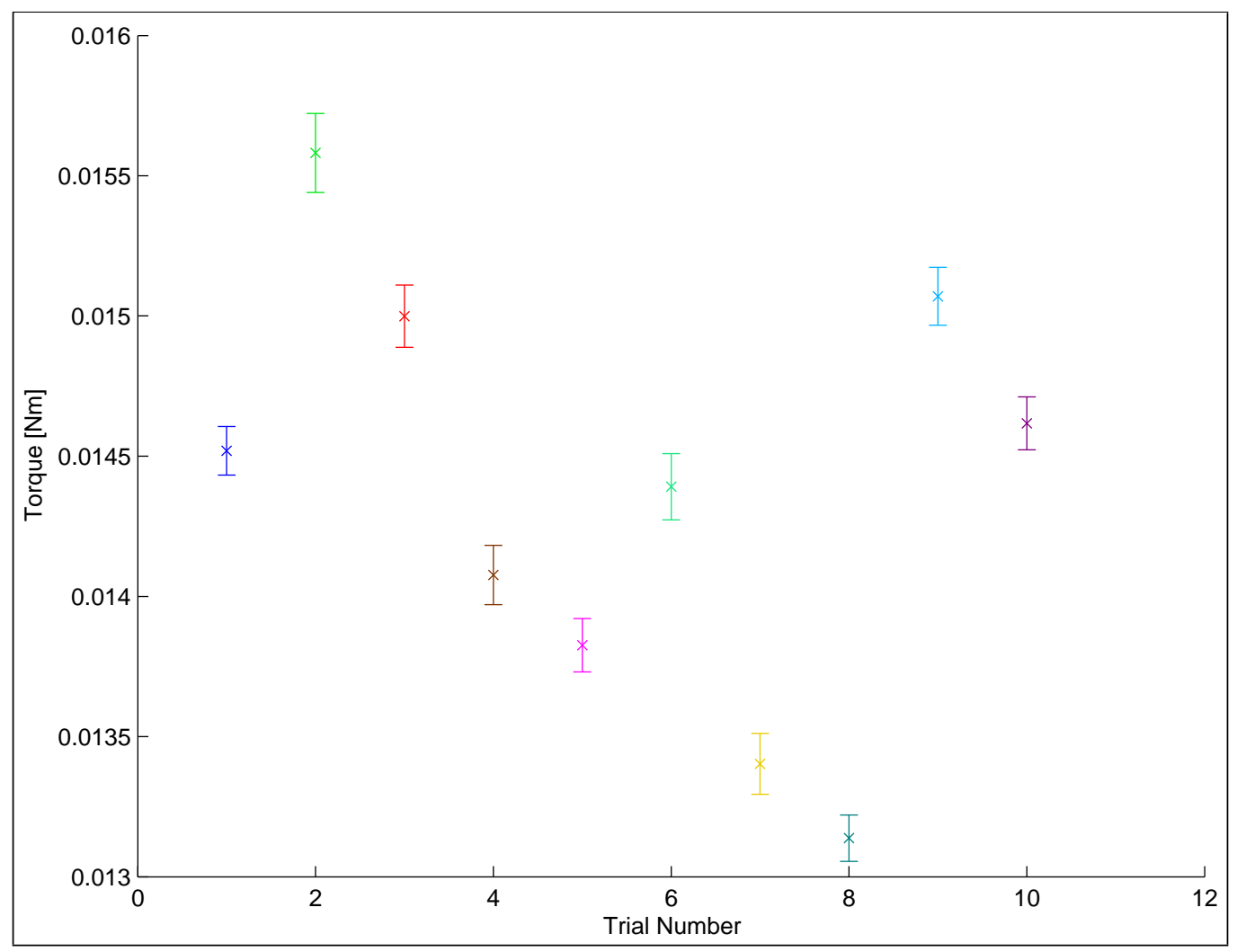

Figure 3.26: Calculated torque measurements for a particular motor speed and current setting. Each point represents the mean torque for a single trial and the error bars show the associated standard error in the torque measurements for that trial. All 10 trials, are plotted to show the spread in measurements between trials and hence the consistency of the damper's operation.

As shown in Figure 3.26 the standard error within a trial is lower than the inter trial variation. Therefore it is possible to adequately characterise variations in damper performance. 


\subsection{Summary}

Using the specified objectives the linear and rotary damper configurations were compared and a rotary damper was selected.

A test rig containing a pulley system was created to measure the output opposing force produced by the various dampers. To minimise unwanted movement and ensure repeatability of the measurements, all the components were aligned and fixed. The design of the text rig was flexible to allow dampers ranging from $55 \mathrm{~mm}$ to $140 \mathrm{~mm}$ in height to be used.

Three different vane designs for the damper were designed and constructed. One of the vanes was the commonly used disc design and the other two were a paddle and helix design.

Computer interface hardware was used to sample and collect force and temperature measurements. A program was integrated with the test rig to display the force and temperature during testing and recorded the measurements for later data analysis.

A description of the data analysis procedure was provided. The force measurements obtained from the test cycles were converted to torque results and statistical analysis was used to find the variation (and hence consistency) of the measurements both within and between trials. 


\section{Chapter 4}

\section{Results}

It was necessary to quantify the performance of the vane design under a number of conditions to determine if the overall system was feasible for a stroke rehabilitation device. Initial experiments using air, water and oil in the damper were used to investigate existing points of friction between the vane and housing and get an indication of whether the vane designs would be suitable to operate as a damper.

An experiment was conducted using homemade MR fluid to determine the controllability and consistency of the damper's response using the three vane designs. The conducted experiment measured the opposing force produced by the vanes as a function of damper coil current. The results of the experiment were used to select the vane design to be further developed.

The paddle design was selected and commercial MR fluid was used to validate the previous results that were obtained using the homemade MR fluid. The settling of both fluids was investigated to determine if it would hinder the performance of the damper. The damper was scaled in size to reduce its mass and increase portability. The effect the size of the damper had on the generated force was investigated. The effect of temperature on the viscous torque was measured to determine how heating of the coil affected the fluid and therefore performance of the damper. 


\subsection{Understanding the behaviour of the damper designs using three different fluids}

An experiment was conducted to measure the opposing force generated by the three vane designs when operating in three different fluids, air, water and oil. The results of the experiment were used to identify pre-existing points of friction and to determine if the vane designs were suitable for use in a MR damper. The experiment conducted with only air in the damper was used to measure the magnitude of existing friction between the vane and housing. Water and oil were used to test if the vane designs caused a change in the generated force of the damper with the known changes in viscosity. The measured force using water and oil allowed an estimate of the force range to be found. This was to be used as a check when using MR fluid to determine whether low measured forces were caused by the design of the vane or whether the viscosity of the fluid was not high enough to provide sufficient damping (i.e. the damper needed more current or coil turns).

There were 10 trials obtained for each of the three fluids and motor speeds.

\subsubsection{Air filled damper results}

Experiments were conducted with only air in the damper to measure the structural friction force occurring between the vane and housing. The motor's speed was varied to investigate how the magnitude of the frictional forces changed with an increase in the rotational velocity of the damper. Where possible modifications to the vanes were made to minimise any identified points of friction. 


\section{Air: Discs design}

The frequency of the spikes in force measurements shown in Figure 4.1 indicate that there was a consistent frictional point that would catch as the shaft rotated. This catching was more pronounced at lower speeds due to the lower momentum of the rotating shaft being insufficient to overcome the friction. The construction of the vane required each rotating disc to be positioned between two stationary discs and separated by washers. As the pieces were 3D printed, although they were filed, there still remained irregularities and roughness of the pieces that contributed to the observed frictional forces. Based on the mean values of the averaged graphs in Figure 4.1 the average frictional force was estimated to be approximately $0.2 \mathrm{~N}$. 


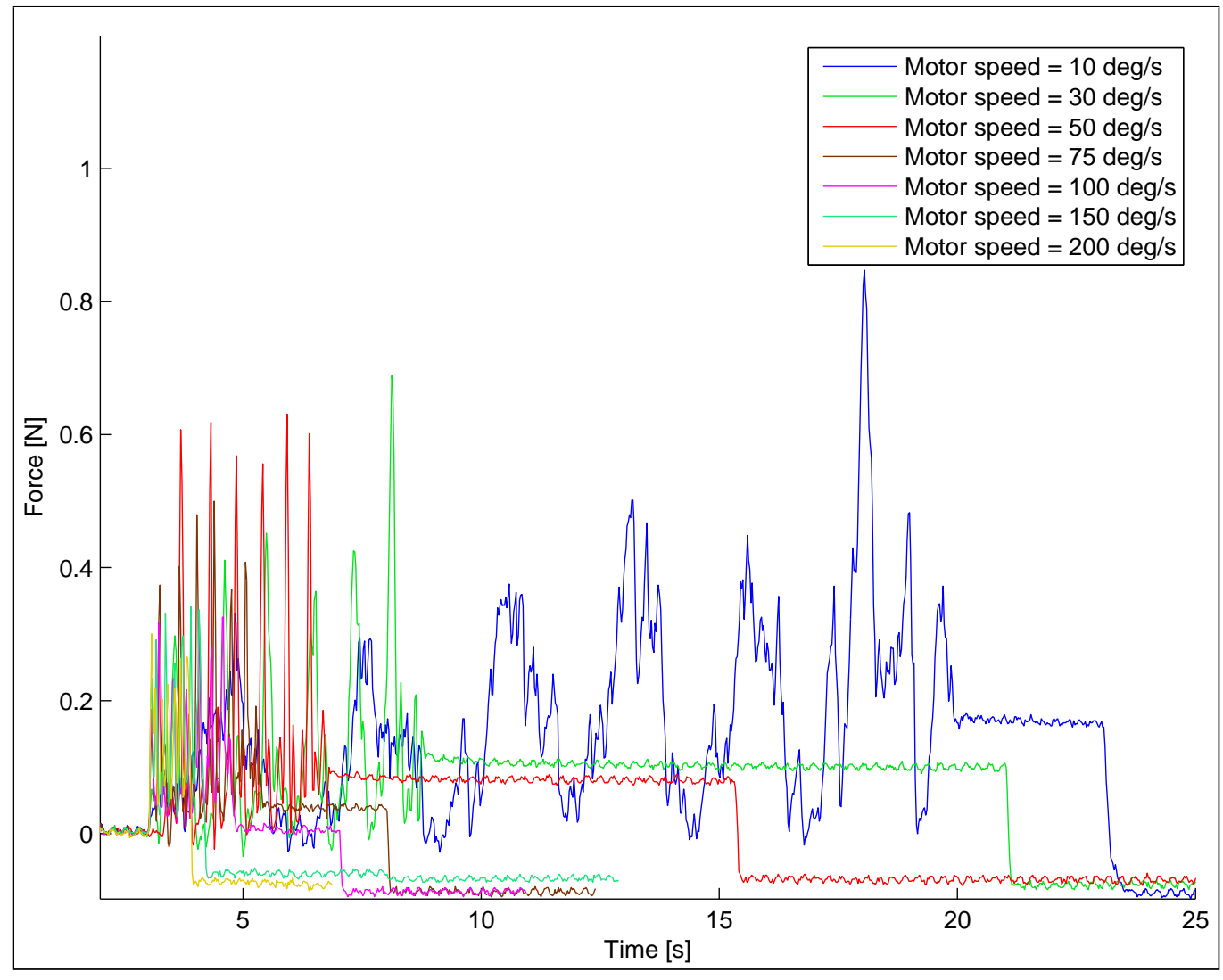

Figure 4.1: The discs vane design rotating inside the air filled damper with varying motor speeds, where each graph represents an average of 10 trials. Note the periodic force measurements indicate a consistent friction point.

\section{Air: Flat paddle design}

There was a spike in the force measurement at the beginning of each test cycle that may have been caused by the static friction between the vane and the housing, which needed to be overcome. It is interesting to note that the change in motor speed did not cause distinctive changes in the generated opposing forces of the damper, which based on the mean of the graphs is estimated to be approximately $0.04 \mathrm{~N}$. 


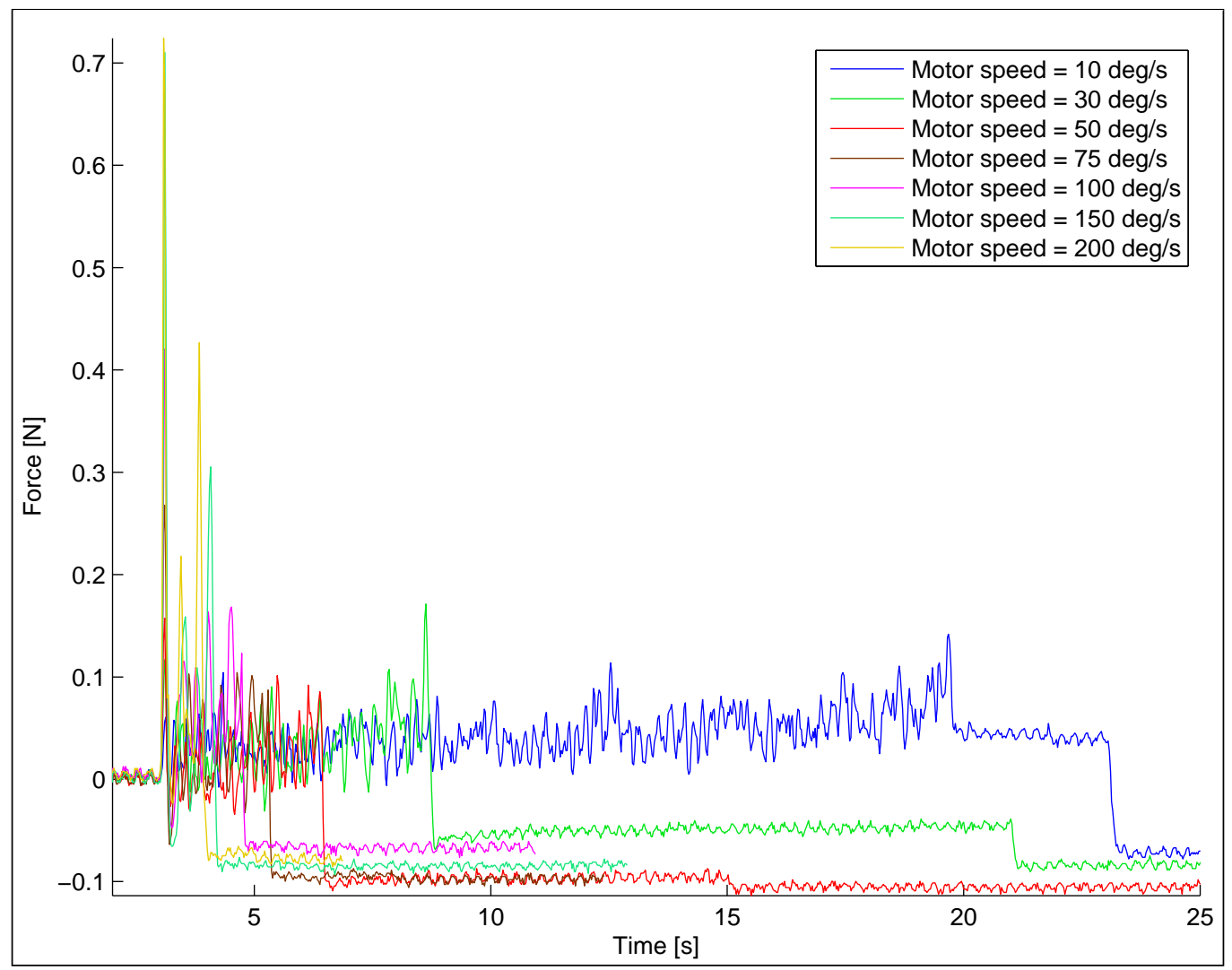

Figure 4.2: The paddle vane rotating inside the air filled damper with varying motor speeds where each graph represents an average of 10 trials.

\section{Air: Helix design}

Similar to the force results of the discs in air, Figure 4.3 indicates that the helix vane also had a consistent frictional point. Some of the friction may have been due to the helix vane sitting flush on the bottom of the housing, therefore there was constant contact between the rotating vane and the housing. Unlike the discs and paddle vane designs, there was a noticeable increase in the measured opposing force with an increase in motor speed. As the shaft of the vane did not sit inside the locater (see Section 3.4.2), the vane was able to move horizontally around its vertical axis causing it 
to contact with the inner wall of the housing. The horizontal movements of the vane may have contributed to the increase in the observed force, as greater rotational speed increased the impact force between the vane and the housing.

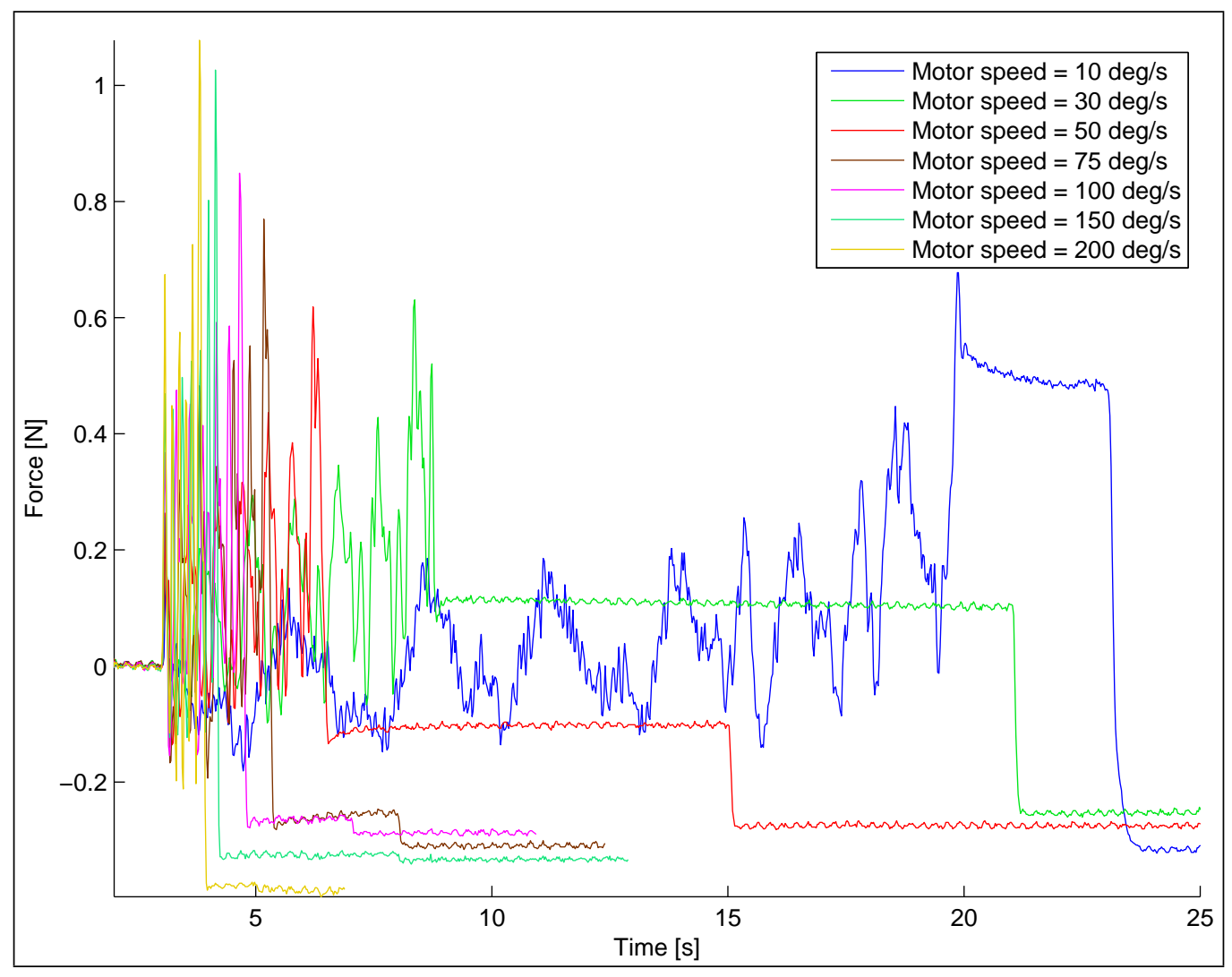

Figure 4.3: The helix vane rotating inside the air filled damper with varying motor speeds where each graph represents an average of 10 trials. Note the periodic force measurements indicate a consistent friction point.

\section{Comparison of vanes in air filled damper}

Since no bearings were used in the housing, it was understandable that there would be frictional forces between the vane designs and the housing. As the discs vane contained many moving parts, there was more op- 
portunity for additional friction to be introduced. Both the discs and helix designs had consistent mechanical sticking that caused periodic spikes in the measured force. The paddle vane produced the lowest estimated averaged force, which may be due to the vane being machined and made from metal. This reduced the irregularities in the shape of the vane and it had a smoother surface in comparison to the other two 3D printed vanes.

However the magnitude of the frictional forces in comparison to the intended output force range of $0-20 \mathrm{~N}$ was still quite low. The experiments containing water and oil will help indicate whether the existing friction in the system could become a problem in later experiments, when MR fluid is used (Section 4.3).

\subsubsection{Water filled damper results}

To observe how the different vane designs interacted in a fluid with low viscosity, the experiment was repeated with the damper filled with water. The force results of the experiment containing the paddle and helix were similar to the magnitude and general trend of the opposing force graphs generated with only air in the damper. The opposing force of the discs design decreased to an estimated average of $0.1 \mathrm{~N}$. This may be due to the water acting as a mild lubricant between the moving parts.

These trials did not uncover anything further of note, so are not discussed further. Plots of the experiment can be found in the Appendix (Section B).

\subsubsection{Oil filled damper results}

Motor oil was used in the damper to observe how the opposing force of the various vane designs responded in a fluid with viscosity similar to MR fluid. It was anticipated that the use of oil may also reduce some of the frictional forces previously seen in the air and water results. To get a better understanding of the consistency of the opposing forces of each 
vane design, the force measurements were converted to torque and the variance in measurements within a trial and the spread between the 10 trials were plotted.

\section{Oil: Discs design}

In comparison to the air and water results for the discs vane, Figure 4.4 shows a noticeable increase in the opposing forces with increases in the motor's speed, with a estimated maximum mean force of approximately $4.5 \mathrm{~N}$ (factoring in the increase in force due to change in pulley radius). The lubrication of the oil appears to have reduced the points of friction that were observed in Figure 4.1. To determine the consistency of the results the change in torque for given motor speeds were plotted, see Figure 4.5. The response of the calculated torque appears linear with changes in motor speed. As the motor's speed increases the variation within a trial and the spread between the trials increases. 


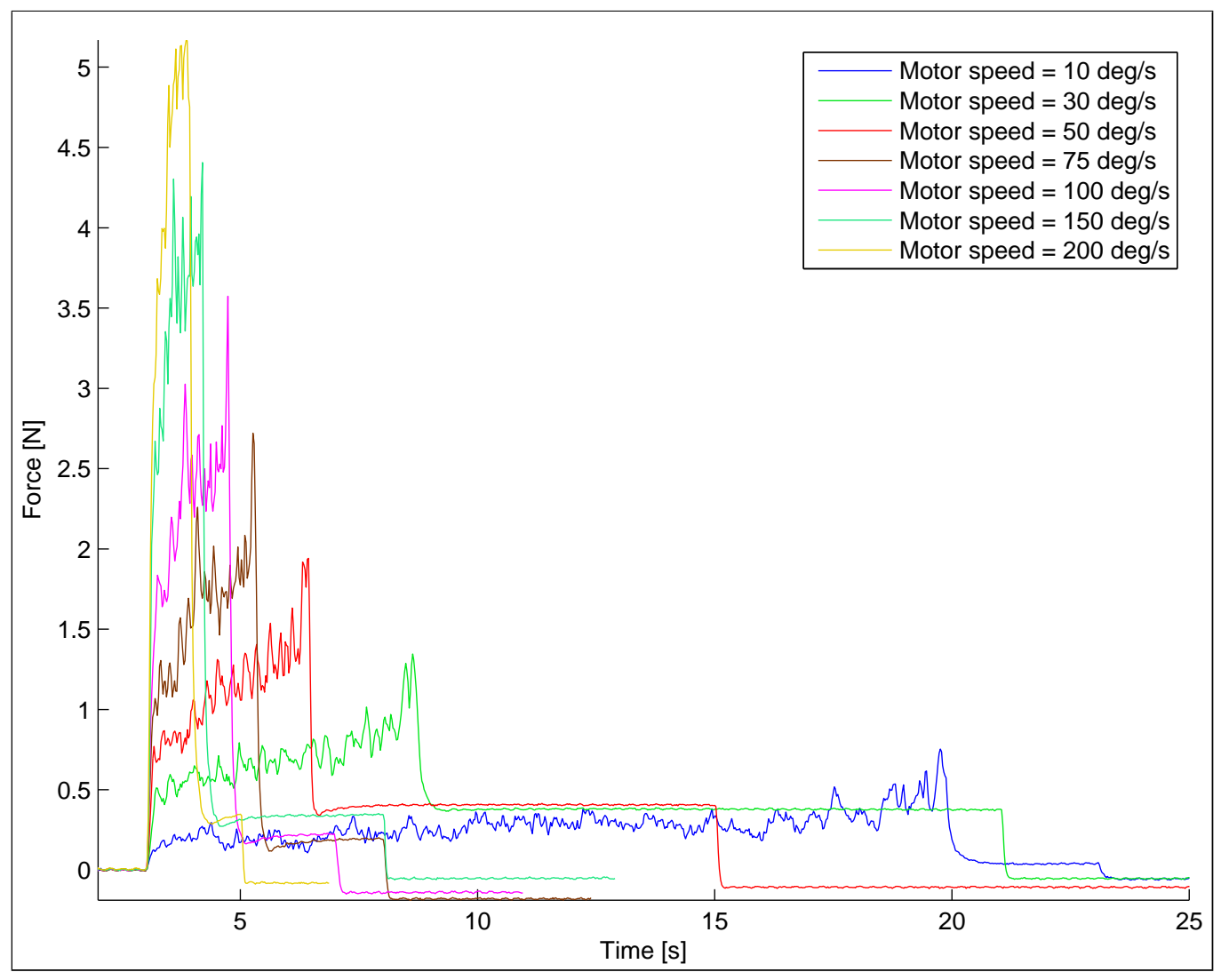

Figure 4.4: The discs vane rotating inside the oil filled damper with varying motor speeds where each graph represents an average of 10 trials. 


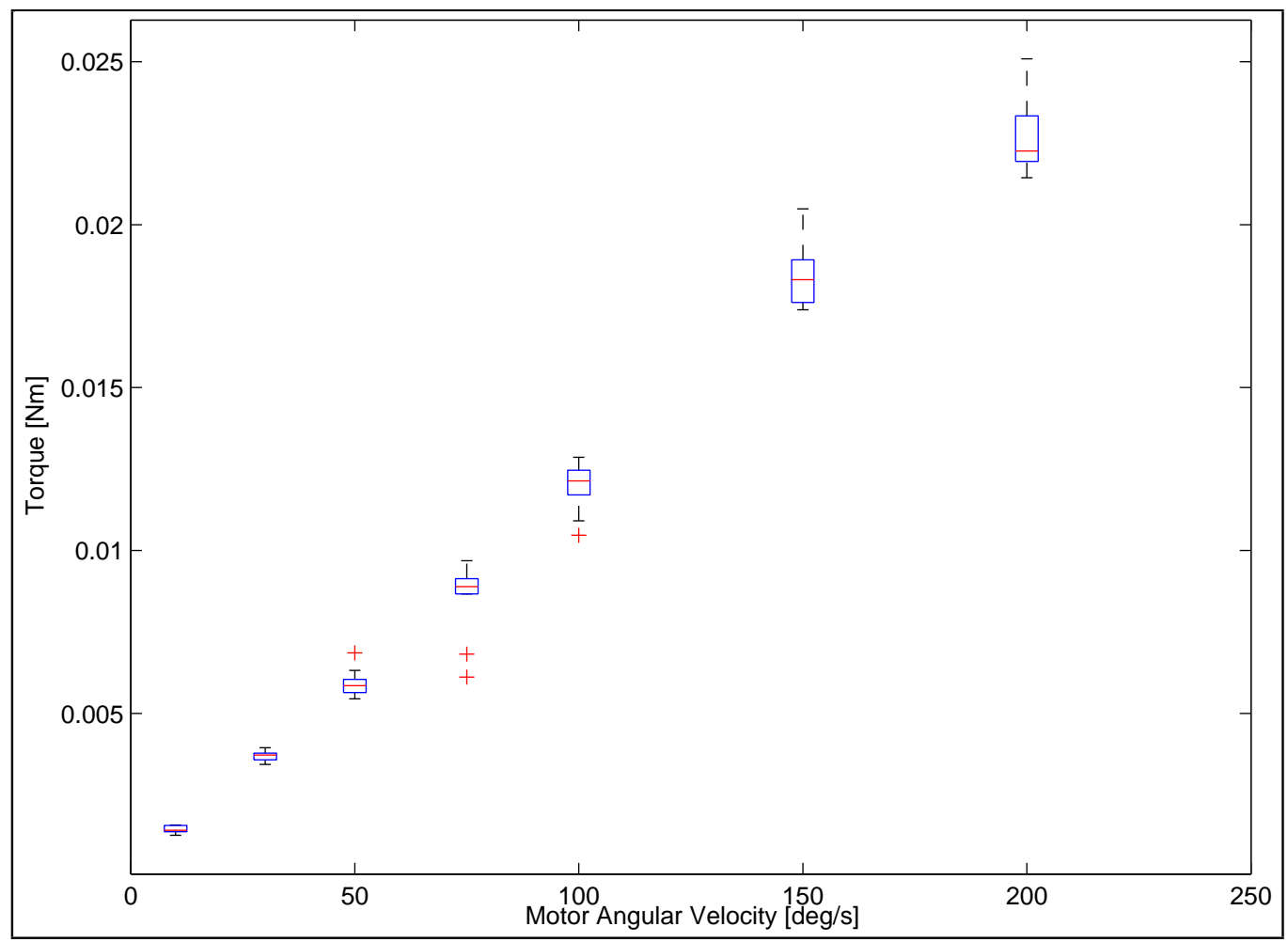

Figure 4.5: Calculated torque measurements of the discs rotating at various motor speeds inside the oil filled damper. There were 10 trials for each of the seven motor speeds. Note the spread in the calculated torque as the motor's speed increases.

\section{Oil: Flat paddle design}

In comparison to the air and water results for the paddle, there is a noticeable increase in the opposing forces shown in Figure 4.6. Considering the change in the radius of the damper's pulley, there is an estimated maximum mean force of approximately $5.5 \mathrm{~N}$. When observing the plot of the calculated torque measurements in Figure 4.7, aside from the measurements at $50^{\circ} \%$, there are no overlaps between the trials of the different motor's speed settings. 


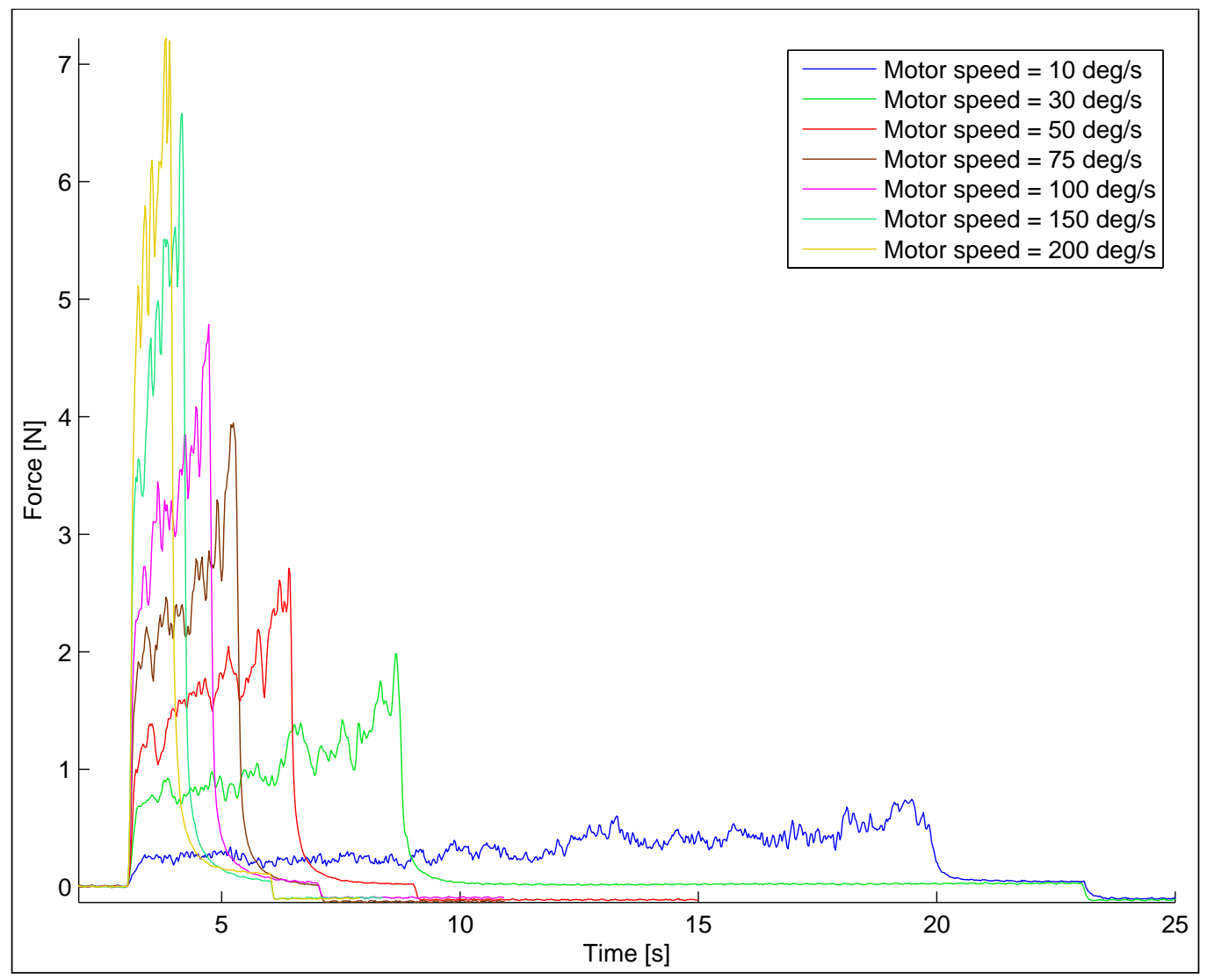

Figure 4.6: The paddle vane rotating inside the oil filled damper with varying motor speeds where each graph represents an average of 10 trials. 


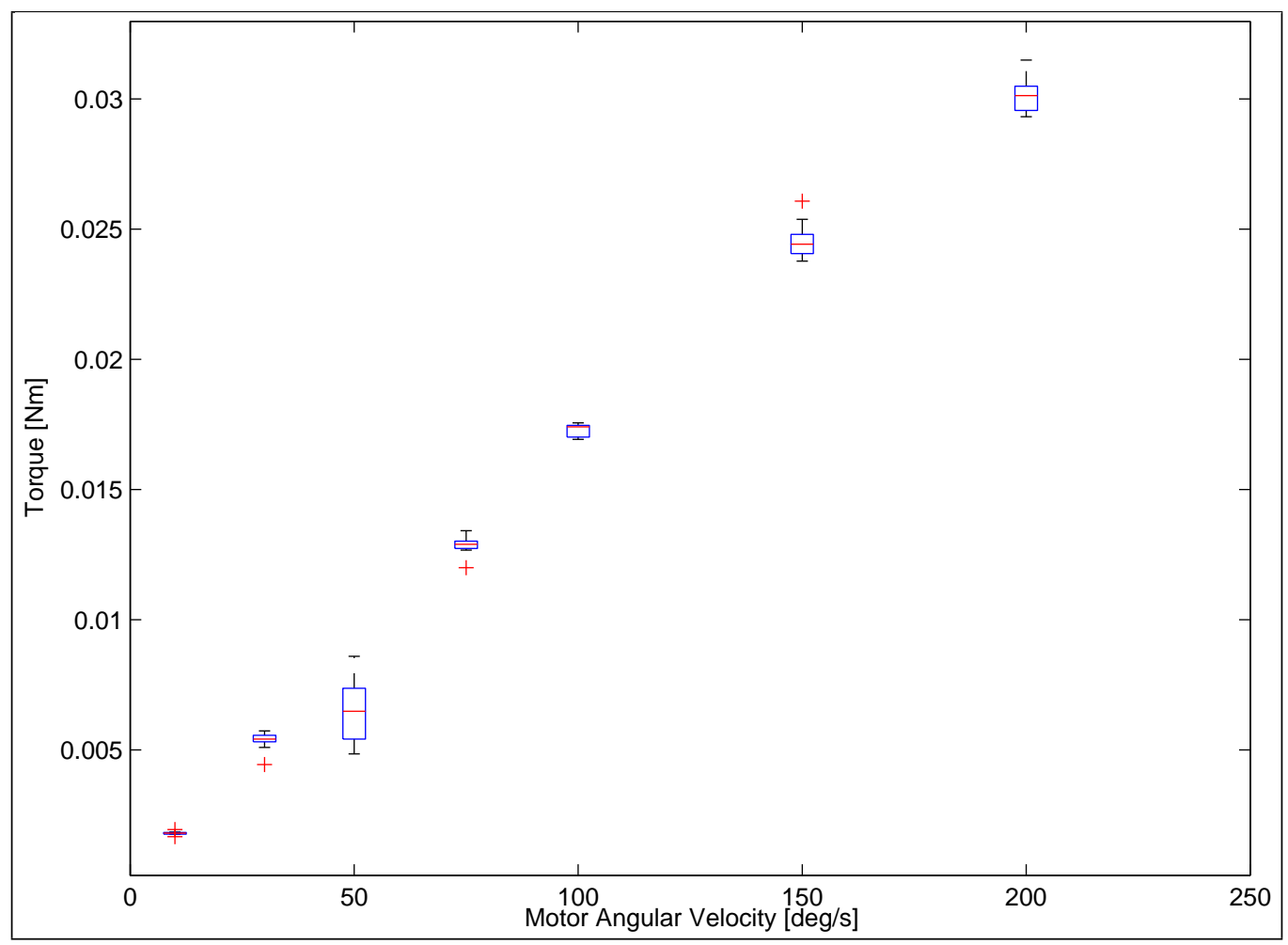

Figure 4.7: Calculated torque measurements of the paddle rotating at various speeds, inside the oil filled damper. There are 10 trials for each of the seven motor speeds.

\section{Oil: Helix design}

It was anticipated that during operation the helix vane would pump the fluid to increase the pressure inside the housing. The measured opposing force of the damper when set at the lowest motor speed of $10^{\circ} / \mathrm{s}$ is shown in Figure 4.8. 


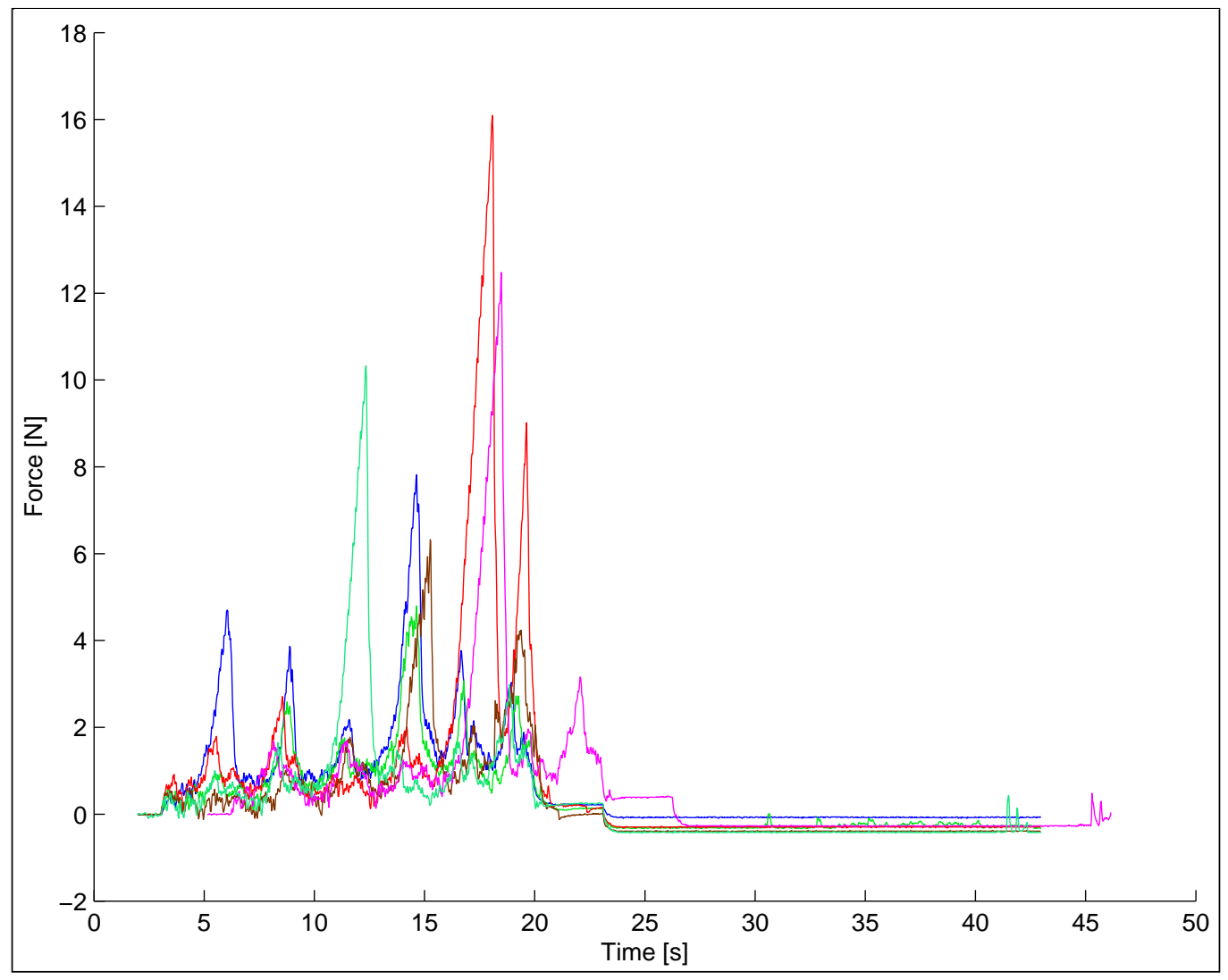

Figure 4.8: Helix vane rotating inside the oil filled damper with a motor speed of $10^{\circ} / \mathrm{s}$. Each graph represents one of the 6 trials. It was hypothesised that the spiking observed in the graph was the result of pressure build up caused by the helix vane pumping the oil.

It was hypothesised that the cause of the force spikes were due to the pressure build up caused by the helix pumping the oil. Therefore three, $3 \mathrm{~mm}$ holes were alternatively drilled vertically, through the helix vane and parallel to the shaft, to provide the fluid with a return path. Using the modified helix, for the same motor speed there was a significant decrease in the force measurement with no visible spiking in the force, shown in Figure 4.9. The results support the hypothesis of pressurisation as the holes were able to equalise the pressure. 


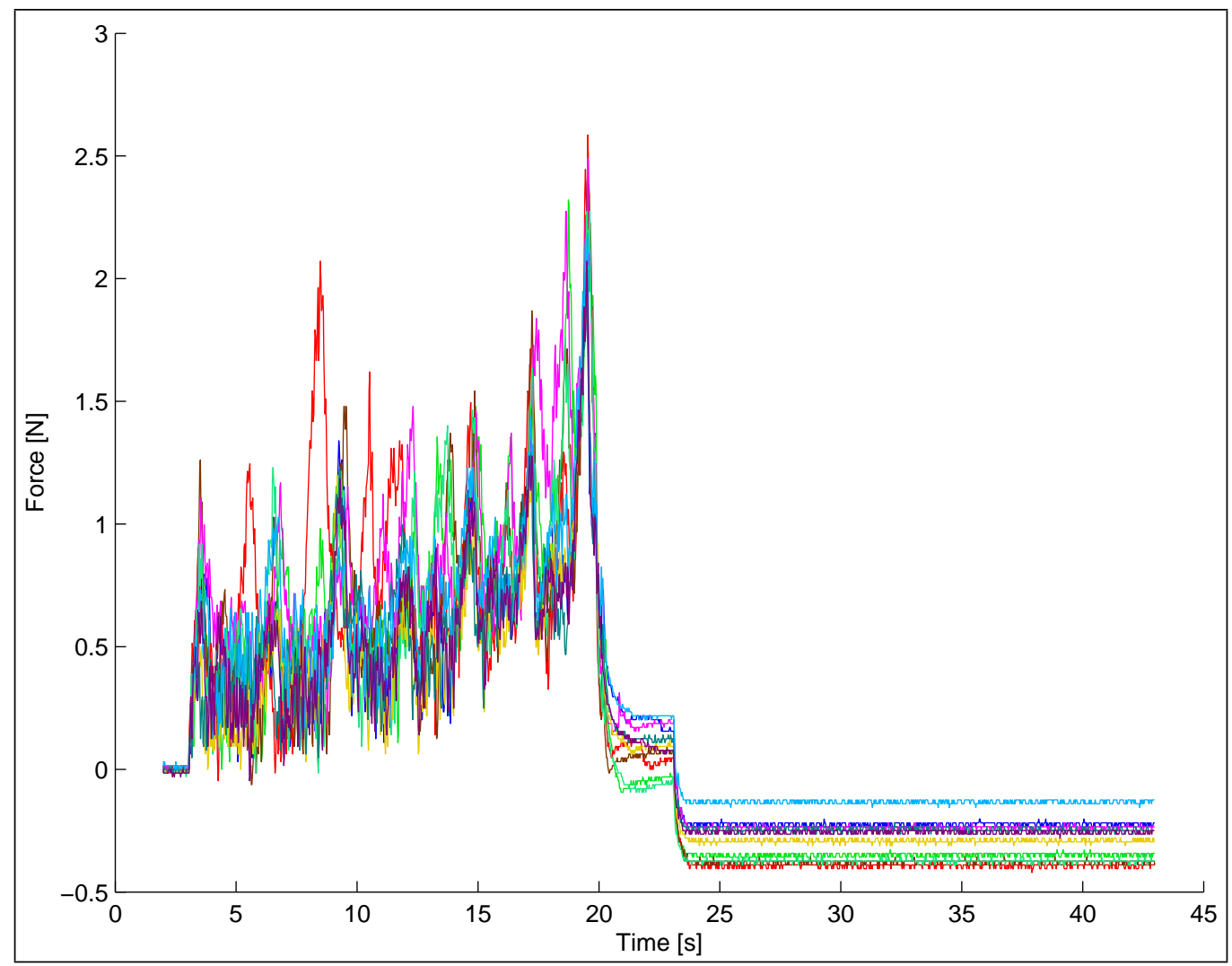

Figure 4.9: The modified helix vane, containing a return path for the fluid, rotating inside the oil filled damper at a motor speed of $10^{\circ} / \mathrm{s}$. Each graph represents one of 10 trials. Note the absence of force spikes of the same magnitude seen in Figure 4.8

There was a periodic oscillation in the force measurements caused by horizontal movements of the vane, about its vertical axis, shown in Figure 4.10. The helix vane produced an estimated maximum mean force of approximately $10 \mathrm{~N}$, with a noticeable increase in the opposing force with an increase in the motor's speed. The inconsistency of the performance of the helix vane is revealed in Figure 4.11. There are large variations in the torque measurements both within a trial and between a set of 10 trials, which increases as the motor's speed increases. At a motor speed of $200^{\circ} / \mathrm{s}$ 
the spread in the calculated torque is approximately $0.018 \mathrm{Nm}$, about $30 \%$ of its total torque range, which would be significant in the control of the damper.

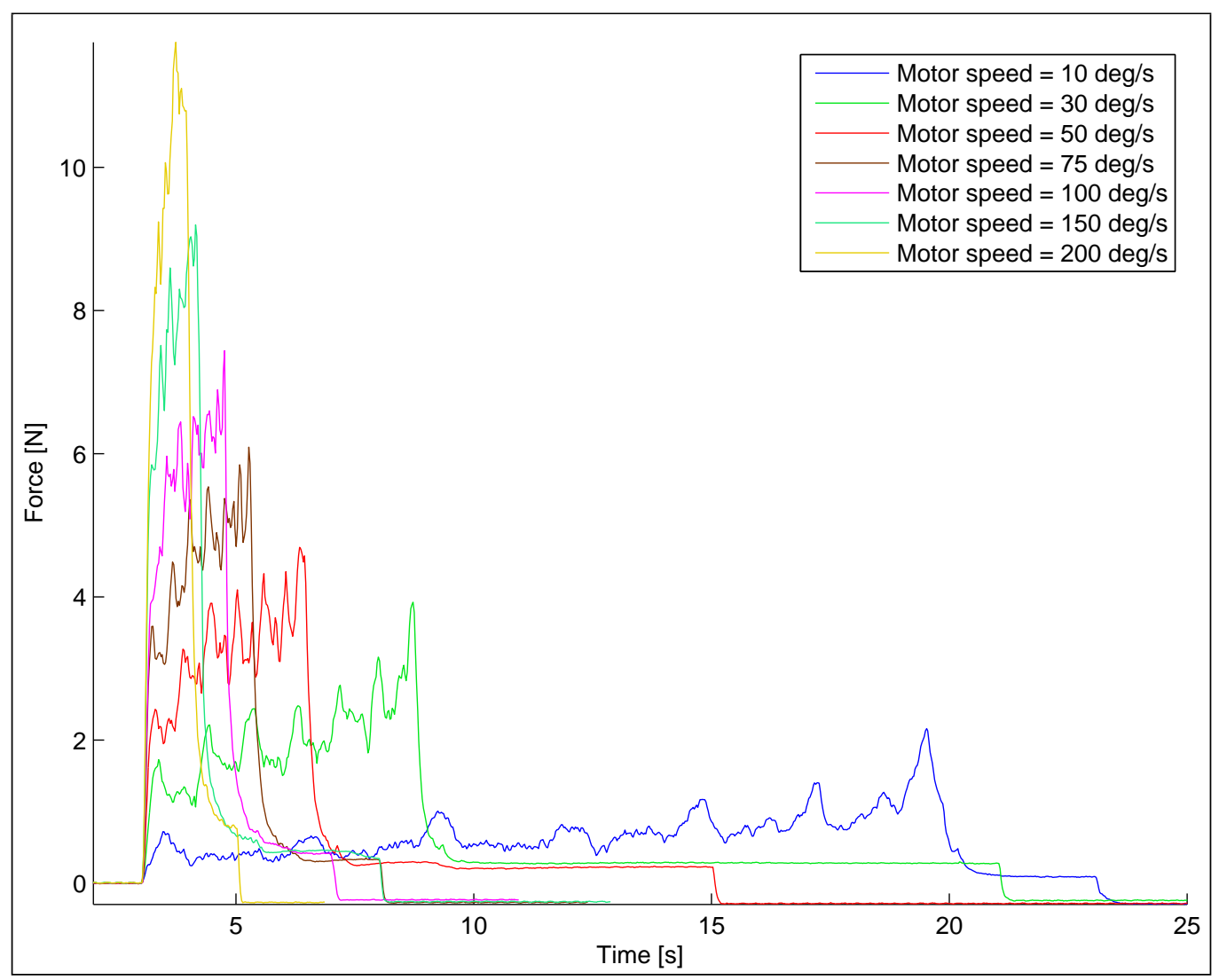

Figure 4.10: The helix vane rotating inside the oil filled damper with varying motor speeds where each graph represents an average of 10 trials. 


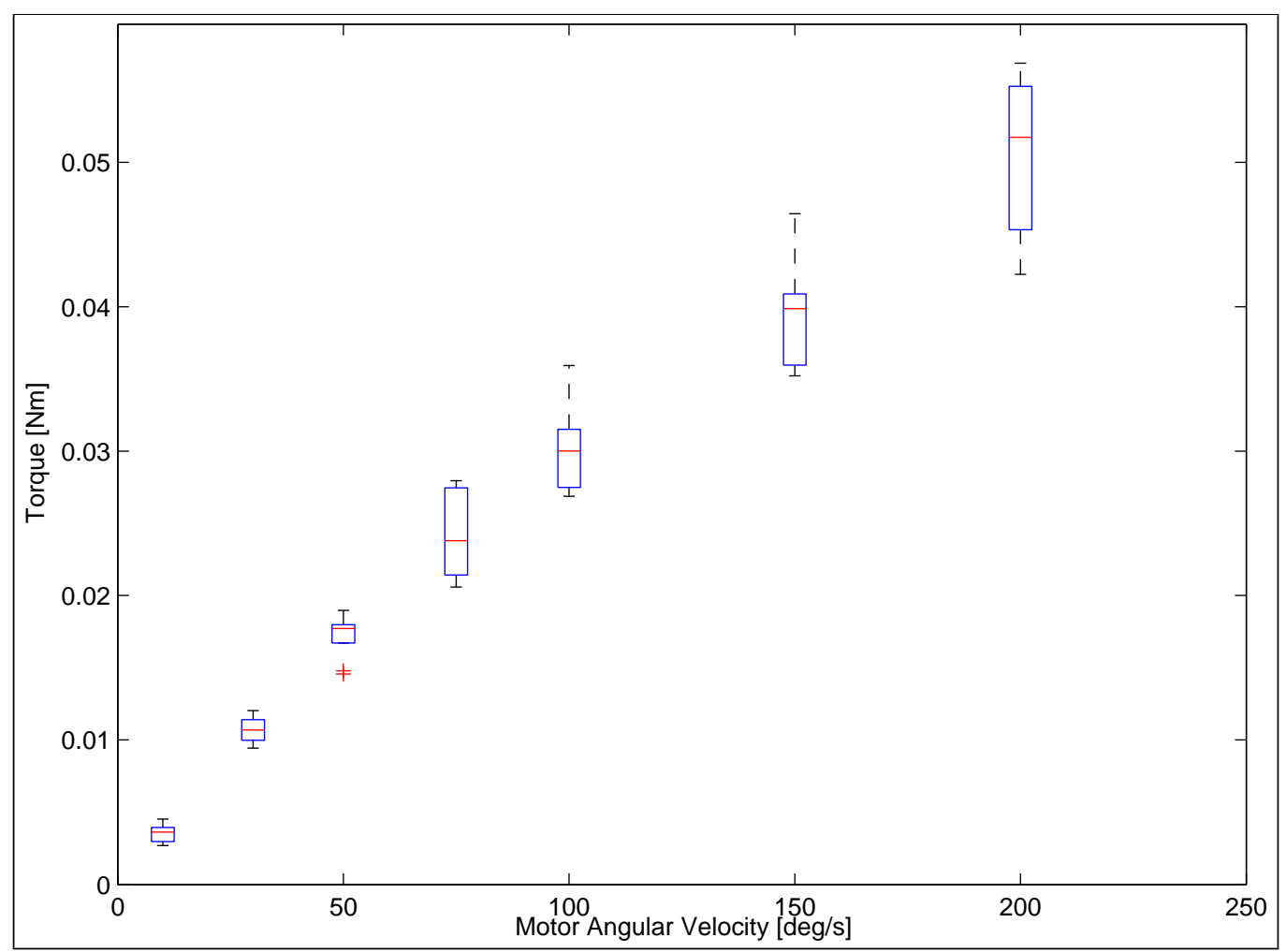

Figure 4.11: Calculated torque measurements of the helix rotating at various speeds, inside the oil filled damper. There are 10 trials for each of the seven motor speeds. Note the spread in calculated torque increases as the motor's speed increases.

\section{Comparison of vanes in oil filled damper}

The oil results showed that with an increase in the viscosity of the fluid, all three vane designs caused an increase in the generated force of the damper. This was a good indication that the vanes were able to be used in an MR damper, where changes in the viscosity of the fluid correspond to changes in the opposing force of the damper. All three vane designs had their range of force within the specified 0-20 N, the helix had the greater generated force approximately $10 \mathrm{~N}$, followed by the paddle at approximately $5.5 \mathrm{~N}$ and the discs with approximately $4.5 \mathrm{~N}$. However when comparing the 
consistency of the results, the calculated viscous torque of the helix varied greatly both within trials and up to an estimated $30 \%$ of the total torque between trials.

\subsection{Temperature Measurements}

Experiments were conducted to determine the acceptable operating temperature range of the PLA plastic and the time response of the heat transfer from the electromagnetic coil to the inside of the housing during operation. Current is applied to the coil to generate the magnetic field needed to change the properties of the MR fluid. As the current is applied the coil heats up, transferring its heat to its surroundings.

Further experiments were required to determine the maximum temperature the $3 \mathrm{D}$ printed parts could tolerate without comprising their shape and strength, and also to determine what the time response of the heat transfer would be.

\subsubsection{Determining a safe operating temperature range of PLA}

An experiment was conducted to determine the safe operating temperature range of the PLA plastic to ensure there was no compromise in shape or strength of the $3 \mathrm{D}$ printed parts during operation. PLA plastic has a rated softening point at $50^{\circ} \mathrm{C}$, however it was important to confirm the temperature at which PLA begins bending with an applied force. A $10 \mathrm{x}$ $150 \times 3 \mathrm{~mm}$ plank of PLA (which had less or equivalent thickness to the parts required for the helix and vane designs) was immersed in water with varying temperatures. A thermometer was used to monitor the temperature. The response of the PLA plank was recorded with changes in applied force (bending it with two hands). The observations of the experiment is shown in Table 4.1. 
Table 4.1: Observed changes to the strength of PLA plastic with changes in temperature

\begin{tabular}{|l|l|}
\hline Temperature ${ }^{\circ} \mathrm{C}$ & Observations \\
\hline$\geq 65$ & The plank bends and morphs on its own \\
\hline $50-55$ & The plank bends easily with little force \\
\hline $40-50$ & The plank can be bent with sufficient force applied \\
\hline$\leq 35$ & The plank holds its shape well \\
\hline
\end{tabular}

From the experiment it was found that to maintain the integrity of the vane and prevent the plastic from morphing, the operating system temperature should not exceed $35^{\circ} \mathrm{C}$. As $35^{\circ} \mathrm{C}$ is above the maximum specified operating temperature of the system, $34^{\circ} \mathrm{C}$ (Section 1.4), there should be no appreciable deformation of the printed helix and discs vane designs during the operation of the system.

\subsubsection{Temperature time response of the system}

An experiment was conducted to estimate the likely change in temperature of the damper containing MR fluid and the time response of the heat transfer from the coil to the inside of the damper. Two LM35 temperature sensors were used to measure the temperature. A fixed sensor under the coil of the damper measured temperature at the heat source and a water proofed LM35 was used to measure the change in temperature inside the damper.

To determine the likely range in time response of the heat transfer, two experiments were conducted. The first experiment measured the temperature change of the inside wall of the brass damper with changes in coil input current, this was to find the fastest time response. Heat paste was used to increase the thermal conductivity between the LM35 and the inner wall. In the second experiment the LM35 was placed in the middle of the damper cavity, which was filled with motor oil. Due to the high iron 
content of MR fluid it was expected that it would likely heat up faster than motor oil, but still slower than then inner wall of the housing. Therefore the results of the two experiments provided a range for the likely change in temperature of the MR fluid.

For each experiment the input current was varied from 0 to $2 \mathrm{~A}$ (the selected range is based on Section 3.1) in increments of $0.5 \mathrm{~A}$. The current was supplied continuously to the electromagnet for over 60 minutes, to simulate the likely operation time of the stroke rehabilitation device. The resulting temperature changes are shown in Figure 4.12 and Figure 4.13. 


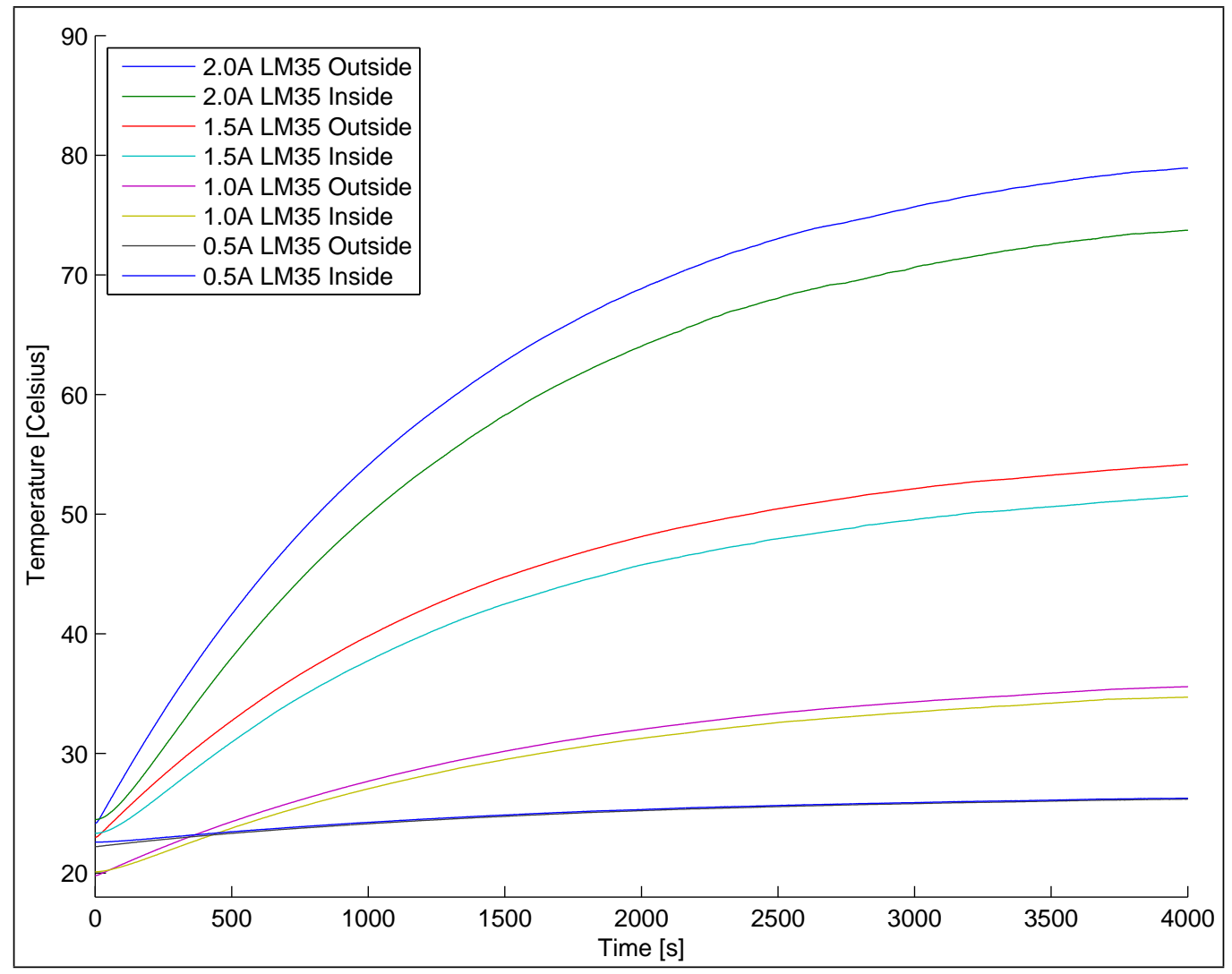

Figure 4.12: Heat transfer from the electromagnet to the inside of the brass housing, in air filled damper. The outside temperature refers to the temperature readings of the LM35 under the coil and the inside temperatures refers to the temperature reading of the LM35 attached to the inside wall of the housing. Note the starting (ambient) temperature for each test is about the same. 


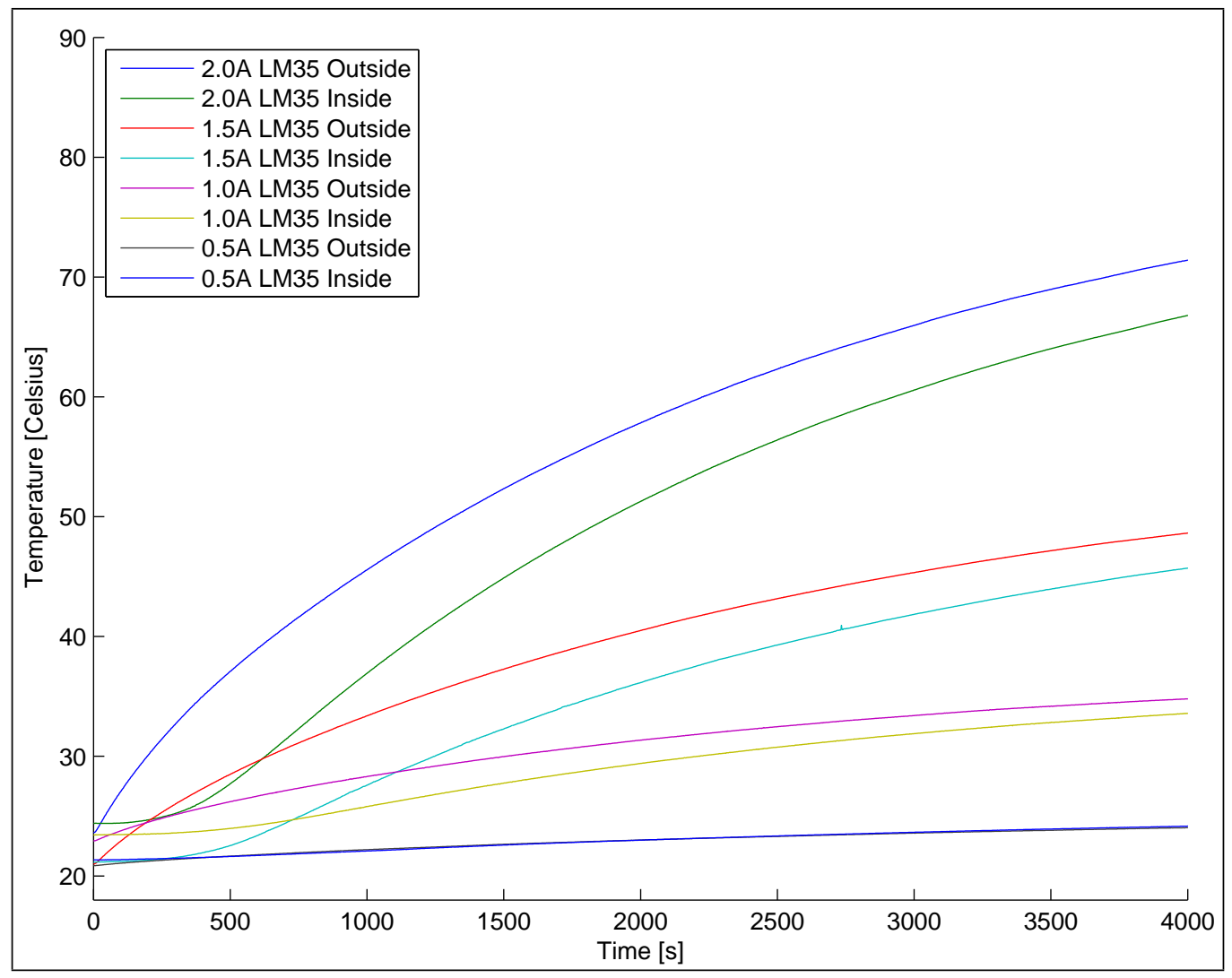

Figure 4.13: Heat transfer from the electromagnet to the motor oil contained in the damper. The outside temperature refers to the temperature readings of the LM35 under the coil and the inside temperatures refers to the temperature reading of the LM35 immersed in the middle of the damper filled with motor oil. Note the starting (ambient) temperature for each test is about the same.

When comparing the outside temperature measurements (readings from LM35 sensor under the electromagnet) the gradient of the graphs in Figure 4.13 are lower than those in Figure 4.12 due to the higher thermal conductivity of oil than air. The heat from the coil is able to be dissipated quicker into the oil than into air. As expected the time delay until changes occur in the temperature, increases as the LM35 is moved further from the 
heat source (from the inside wall results to the oil results). An important finding from the experiment is the steady state temperature values, in the worst case the temperature nears $80^{\circ}$ at 2 A (Figure 4.12).

The longest duration of one of the subsequent damper test cycles was $83.5 \mathrm{~s}$, when the motor speed was set to $5^{\circ} / \mathrm{s}$ (Section 4.4.4). This was still below the shortest time taken for the coil to heat up to $34^{\circ} \mathrm{C}$, which was $266 \mathrm{~s}$ when using the temperature readings of the coil and $365 \mathrm{~s}$ using the temperature readings from the inner wall of the damper. These time durations are the temperature change from ambient temperature with a current of 2 A.

The results indicate that each test is unlikely to be compromised by temperature induced changes in fluid viscosity or temperature changes causing appreciable deformation of the printed helix and discs vane designs. However, the results illustrate the need to allow the damper to cool down between tests.

\subsection{Understanding the behaviour of the damper designs using MR fluid}

To select a vane design, an experiment using MR fluid was performed to determine the controllability and consistency of each design. The conducted experiment measured the minimum and maximum opposing force produced by the vane designs with changes to the input current of the damper. The upper speed limit of the motor was reduced from $200^{\circ} / \mathrm{s}$ to $75^{\circ} / \mathrm{s}$ as this corresponded to the likely maximum speed of the user's interaction. The results of section 4.1 showed that the opposing force generated by each vane design increased with an increase in the motor's speed. Therefore it was assumed that the possible range of forces would be within the upper and lower speed limits of $75^{\circ} / \mathrm{s}$ and $10^{\circ} / \mathrm{s}$. Motor speeds of only $75^{\circ} / \mathrm{s}$ and $10^{\circ} / \mathrm{s}$ were used for this experiment and the input current range 
was between 0 and $2 \mathrm{~A}$. As it was unknown how the opposing force would vary with changes in the current of the damper, input currents at $1 \mathrm{~A}$ and $1.5 \mathrm{~A}$ were also tested so that any non-linear behaviour could be identified.

The order of data collection was conducted similarly to how the damper would operate in the stroke rehabilitation device, many repetitions at the same current setting. For each motor speed all 10 trials were taken consecutively, beginning at the lowest current setting to the highest. At the beginning of each test cycle, the current was turned on after the force sensor was zeroed during the one second wait period. This was to ensure any increased tension in the cord caused by the change in viscosity of the MR fluid, was not zeroed during the force sensor's calibration period. To prevent the damper from overheating the current was turned off between trials and if the temperature measurement of the electromagnetic coils was measured to be above $33^{\circ} \mathrm{C}$, testing was paused to allow the damper to cool down.

The results of the opposing force measurements and calculated torque measurements for each of the vanes designs are presented. The measured opposing force with changes in input current and motor speed, are used to quantify the generated range of force for each vane design in MR fluid. The torque measurements were calculated and are presented with changes to the input current to show the spread in results and therefore the consistency of the damper design.

Because of its cost of MR fluid and the difficulty of purchasing small quantities, the MR fluid used in these tests was made using an obtained online recipe [40]. The recipe is as follows:

- 55 g Oil (3-in-1)

- $5 \mathrm{~g}$ White lithium

- 150 g Iron powder 


\subsubsection{Force results for the discs design in MR fluid}

At first inspection, the damper appears controllable as increases in input current cause distinct increases in the opposing force of the damper shown in Figure 4.14 and Figure 4.15. The controllability of the damper will be furthered investigated when analysing the spread in the calculated torque measurements in Section 4.3.4.

At the slower motor speed of $10^{\circ} / \mathrm{s}$ there was a consistent friction point that occurred at each rotation of the shaft. The resultant spikes in the force become more dominant with an increase in current. This is due to the increase in the fluid's viscosity making it more difficult for the vane to both shear the fluid and overcome the friction point. At the higher rotational speed of $75^{\circ} / \mathrm{s}$ the damper contains greater momentum and is able to overcome the sticking point, as shown in Figure 4.15 where the spikes in the force are not visible.

An estimate of the force range of the vane design, (not including the force spikes as this is caused by the construction of the vane and not the design of the vane) would be between $0.2 \mathrm{~N}$ and $3 \mathrm{~N}$ for the motor's speed of $10^{\circ} / \mathrm{s}$ and $0.8 \mathrm{~N}$ to $4.5 \mathrm{~N}$ for a speed of $75^{\circ} / \mathrm{s}$. 


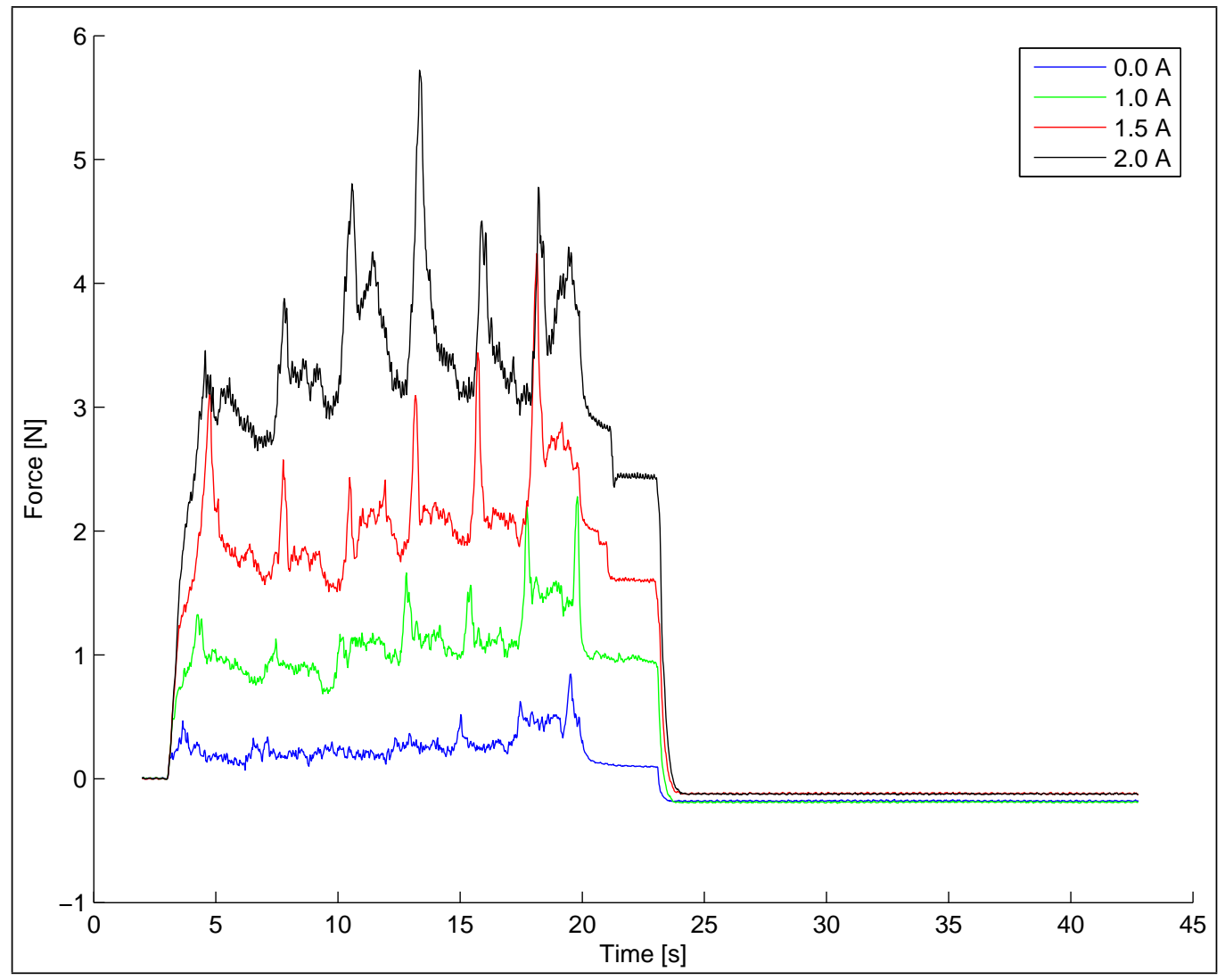

Figure 4.14: The discs vane rotating inside the damper filled with MR fluid with a change in the input current. The motor's speed was set to $10^{\circ} \mathrm{s}$ and each graph is an average of 10 trials. Note the spikes in the force caused by a sticking point in the rotation. 


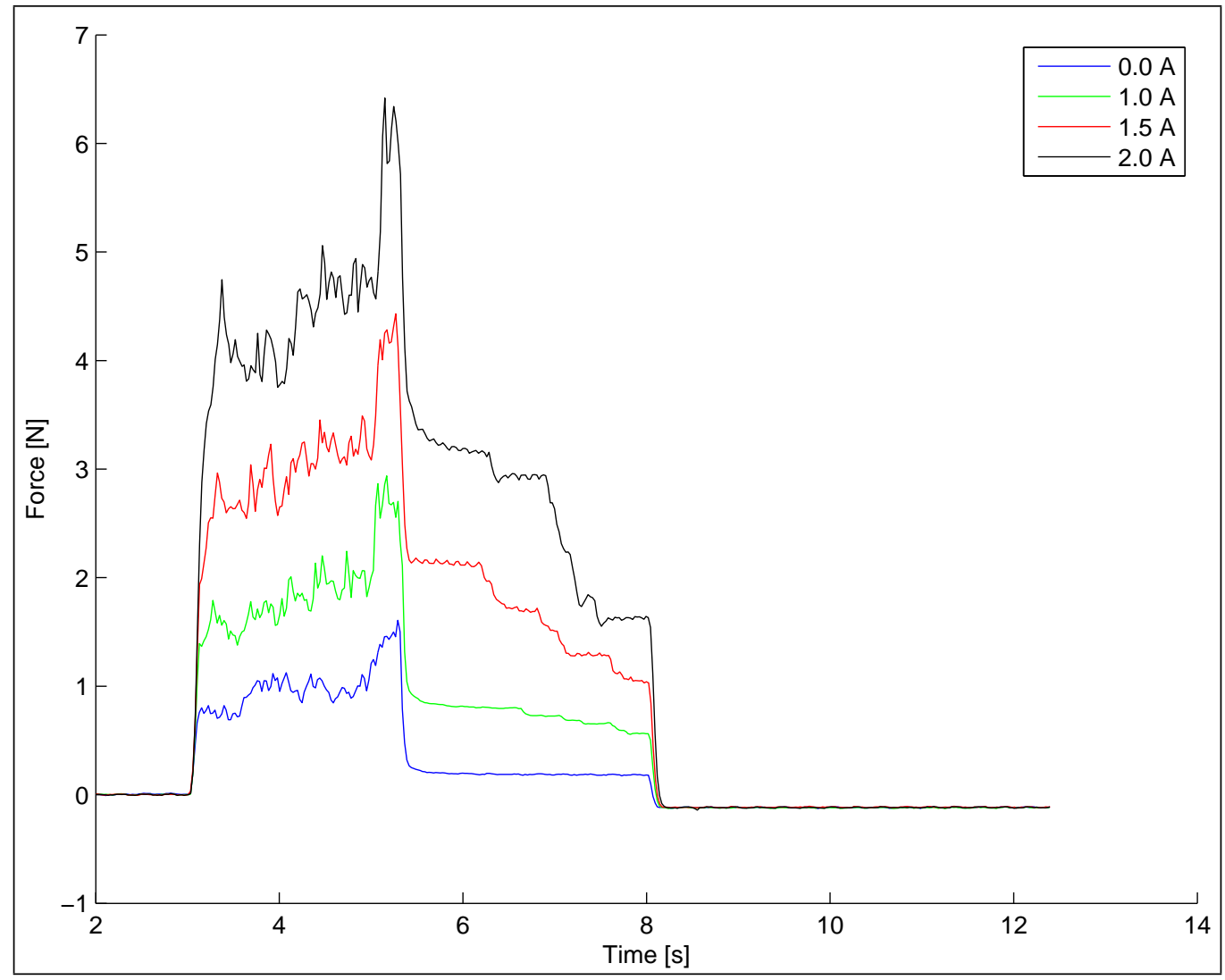

Figure 4.15: The discs vane rotating inside the damper filled with MR fluid with a change in the input current. The motor's speed was set to $75^{\circ} / \mathrm{s}$ and each graph is an average of 10 trials. Note the absence of the dominant spikes in the force observed in Figure 4.14.

\subsubsection{Force results for the paddle design in MR fluid}

Similar to the results of the discs design (Figure 4.14), there are distinct differences in the measured force with changes in the input current, indicating the potential to control the damper.

As the housing only contained a locater at the bottom to guide the shaft, the paddle was able to wobble away from its ideal vertical axis. As the input current was increased, it was easier for the shaft to move in an el- 
liptical path about its vertical axis than it was to rotate around the vertical axis and move the vane through the higher viscosity fluid. This horizontal movement caused oscillations in the measured force. Shown in Figure 4.16 there are six major peaks in the force, which corresponds to the number of rotations of the damper. Each of the peaks contain two smaller peaks, due to the elliptical movement of the shaft. At a motor speed of $10^{\circ} / \mathrm{s}$ the approximated $6 \mathrm{~N}$ opposing force range is over twice the measured force range of the discs design with the equivalent speed.

With reference to Figure 4.17 the unexpected increase in the averaged force graph of $1.5 \mathrm{~A}$ at approximately five seconds, is due to an outlier in the trials, which had a peak force of $24 \mathrm{~N}$ and was caused by the shaft getting stuck while rotating. The presence of the outlier is seen in Figure 4.21 . 


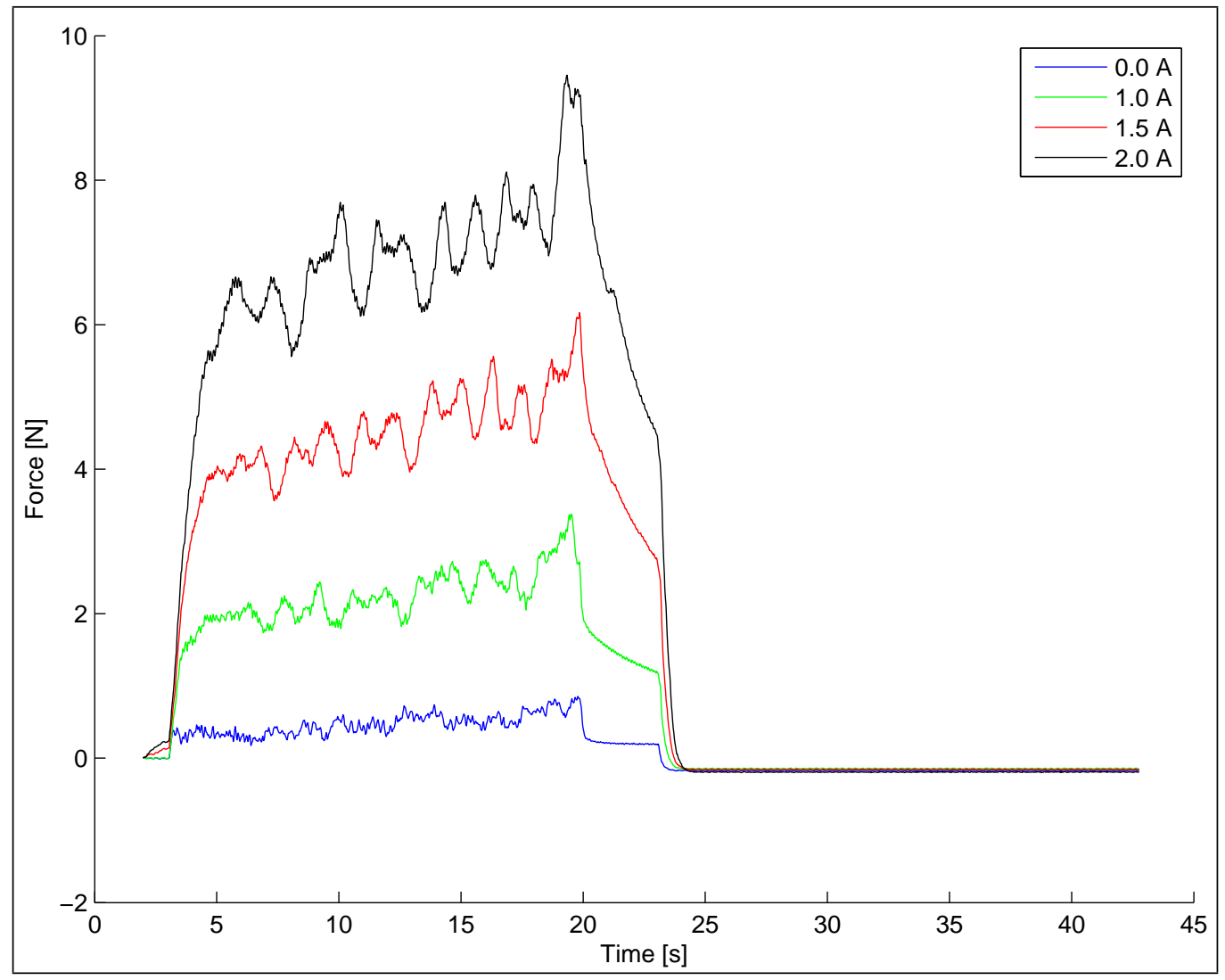

Figure 4.16: The paddle vane rotating inside the damper filled with MR fluid with a change in the input current. The motor's speed was set to $10^{\circ} / \mathrm{s}$ and each graph is an average of 10 trials. Note the periodic spikes in the measured force due to the elliptical movement of the shaft about its vertical axis. 


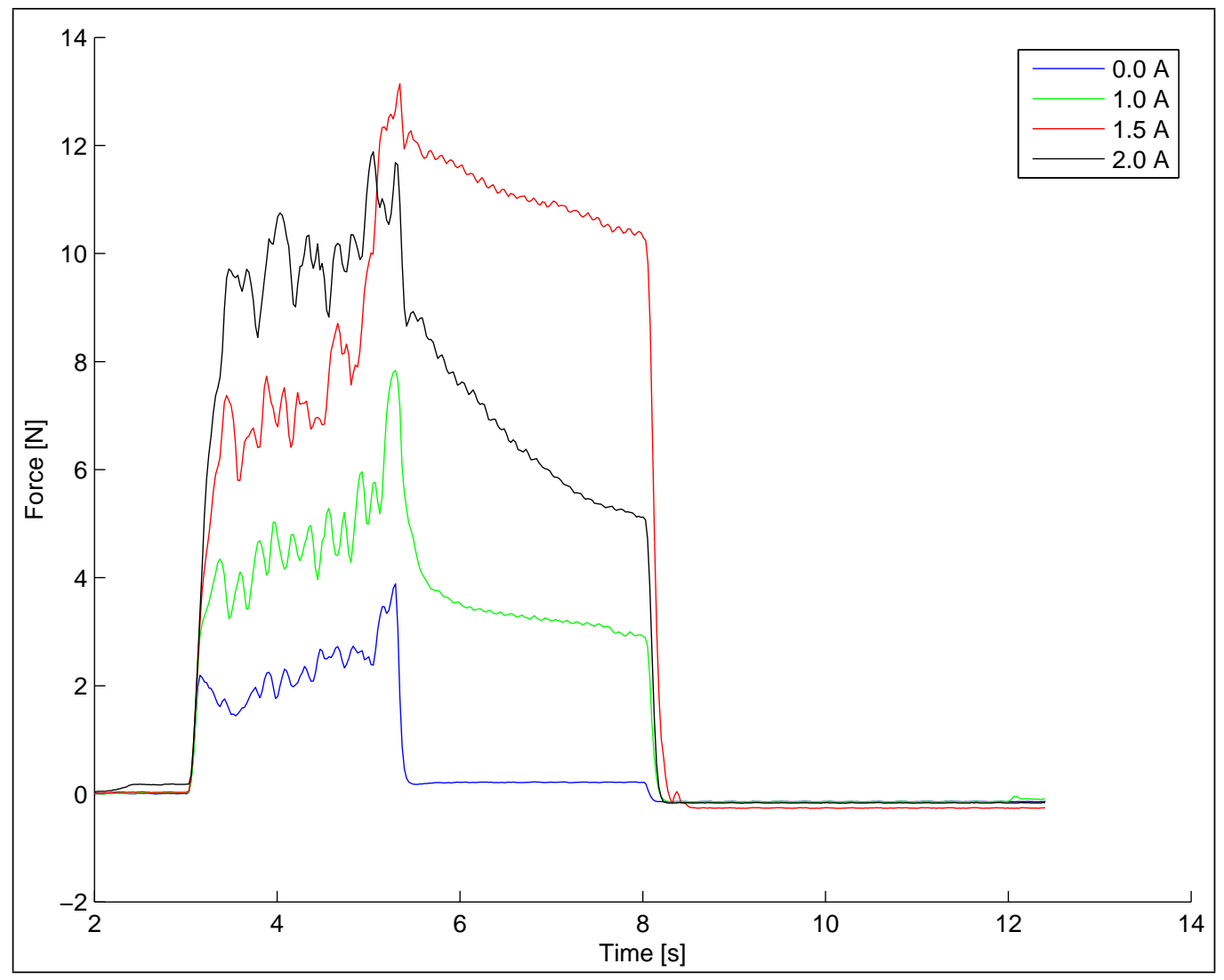

Figure 4.17: The paddle vane rotating inside the damper filled with MR fluid with a change in the input current. The motor's speed was set to $75^{\circ} / \mathrm{s}$ and each graph is an average of 10 trials. Note the unexpected force increase in the graph of $1.5 \mathrm{~A}$ at approximately five seconds, due to an outlier in the results

\subsubsection{Force results for the helix design in MR fluid}

At times the bottom face of the helix vane would catch near the base of the inner wall of the housing. This may have been due to the irregular inner diameter of the brass tubing. To reduce this problem the vane was elevated by a couple of millimetres by modifying the shaft. This reduced the occasional spike in the force, previously seen in the air results. Similar 
to the paddle design, the shaft of the helix vane also moved in an elliptical path around its vertical axis. The periodic oscillations can be seen in Figure 4.19.

The range in opposing force of the helix was greater than the paddle and discs design. The helix had an estimated mean force range of 1-12 N with a motor speed of $10^{\circ} / \mathrm{s}$ and $2-18 \mathrm{~N}$ with a motor speed of $75^{\circ} / \mathrm{s}$, (discounting the periodic spike in force measurements as the spikes were not caused by the design of the vane).

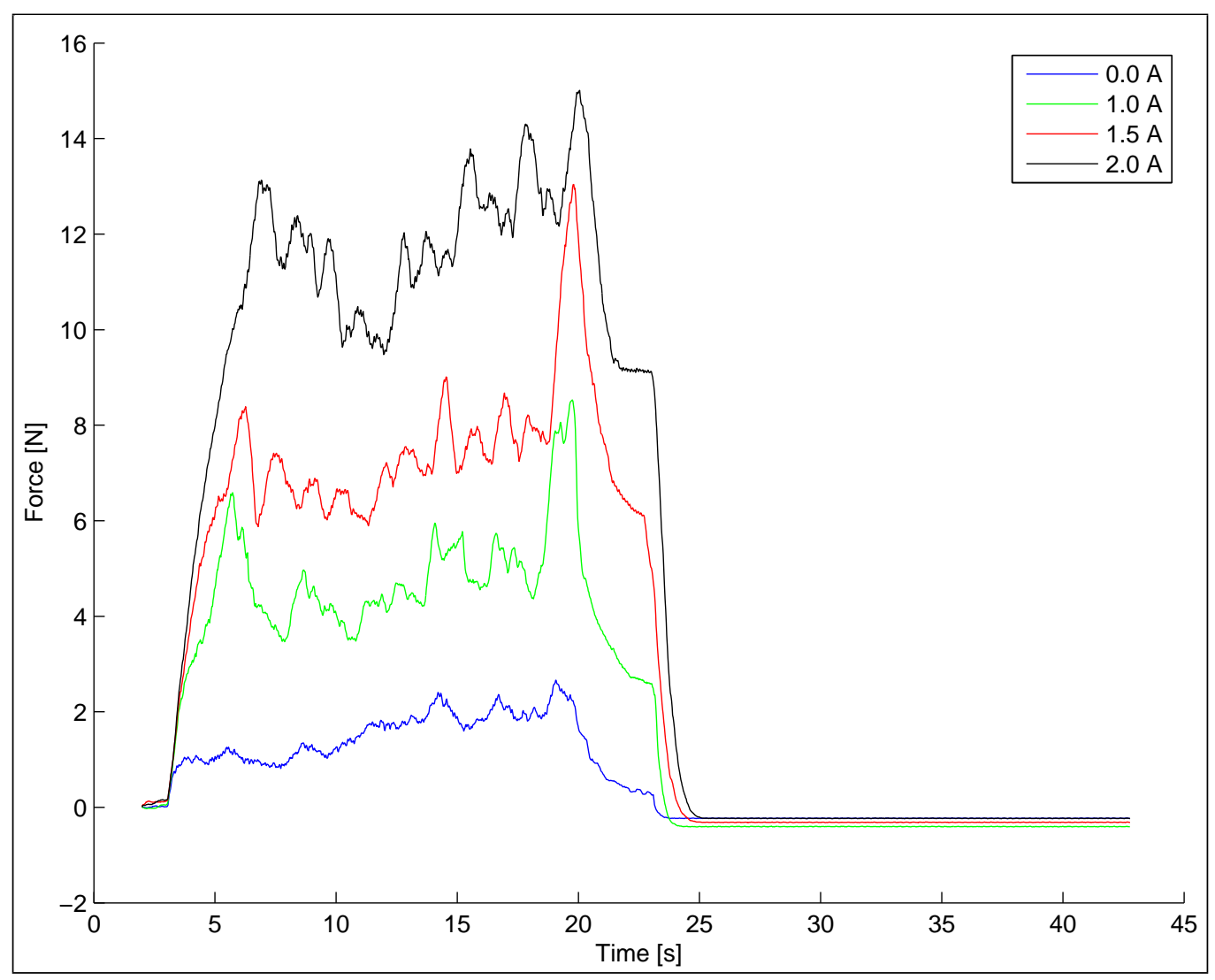

Figure 4.18: The helix vane rotating inside the damper filled with MR fluid with a change in the input current. The motor's speed was set to $10^{\circ} / \mathrm{s}$ and each graph is an average of 10 trials. Note minor spikes in the measured force due to the elliptical movement of the shaft about its vertical axis. 


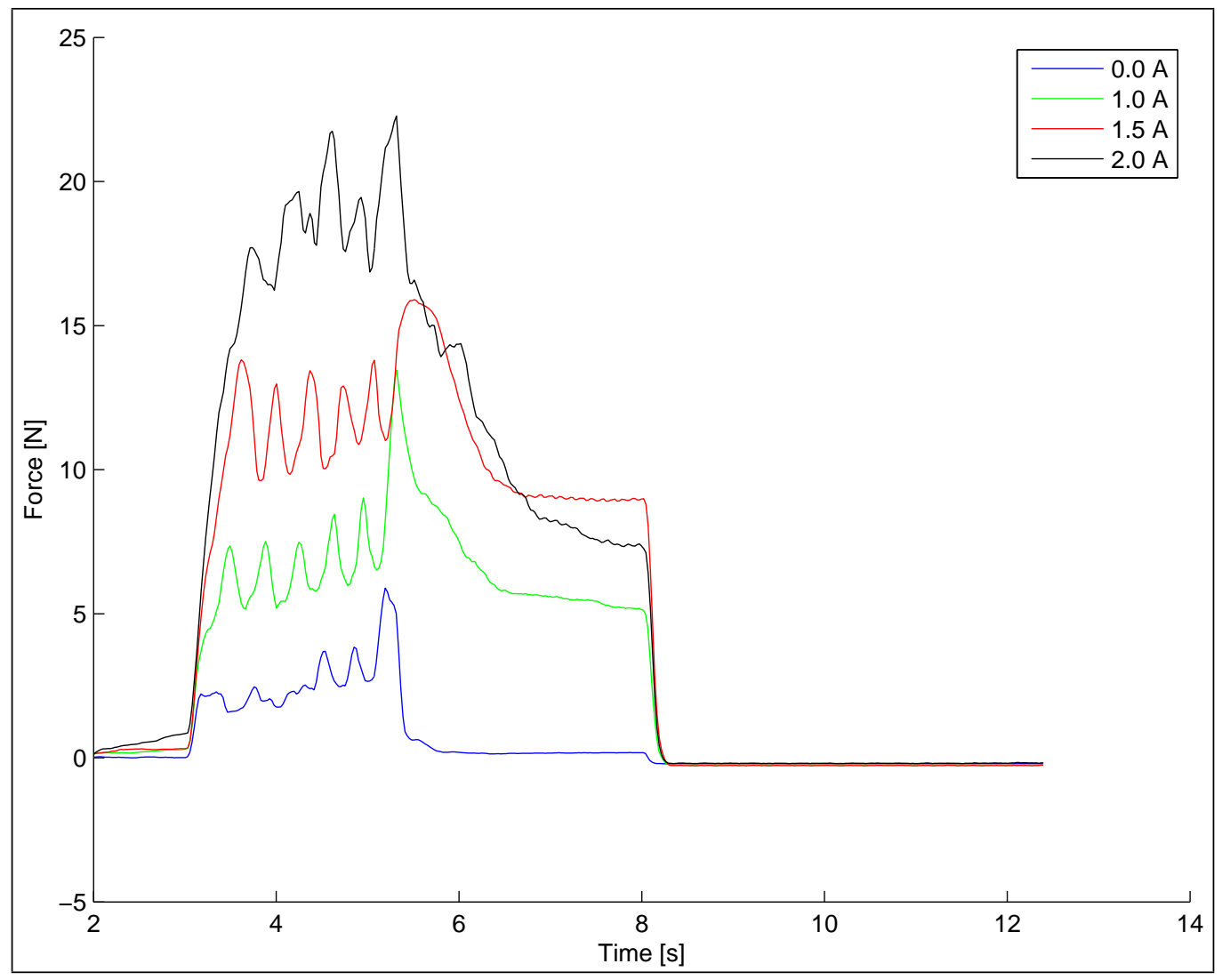

Figure 4.19: The helix vane rotating inside the damper filled with MR fluid with a change in the input current. The motor's speed was set to $75^{\circ} / \mathrm{s}$ and each graph is an average of 10 trials. Note dominant spikes in the measured force due to the elliptical movement of the shaft about its vertical axis.

\subsubsection{Calculated torque of the three vane designs with changes in input current}

The viscous torque was calculated and plotted to examine the consistency of the vane designs by showing the spread in the measurements for each trial. The plots of the calculated torque measurements with changes in the input current are shown as follows: discs design (Figure 4.20), pad- 
dle design (Figure 4.21) and helix design (Figure 4.22). Note the differences in torque scale between the plots.

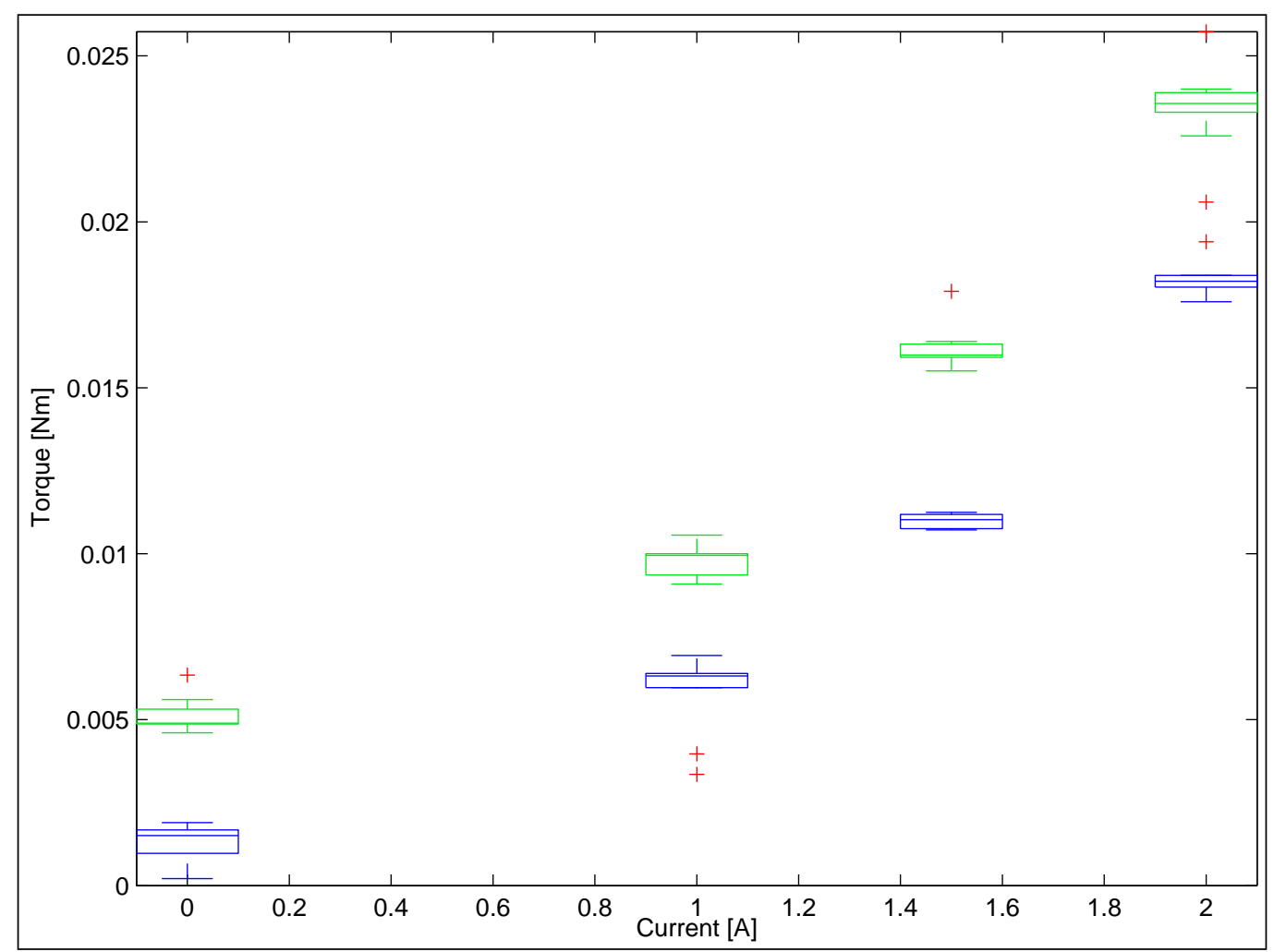

Figure 4.20: Calculated torque measurements of the discs rotating at motor speeds of $10^{\circ} / \mathrm{s}$ (blue) and $75^{\circ} / \mathrm{s}$ (green), inside damper filled with MR fluid. The current was varied for each speed and the plot contains the 10 trials for each of the seven motor speeds. 


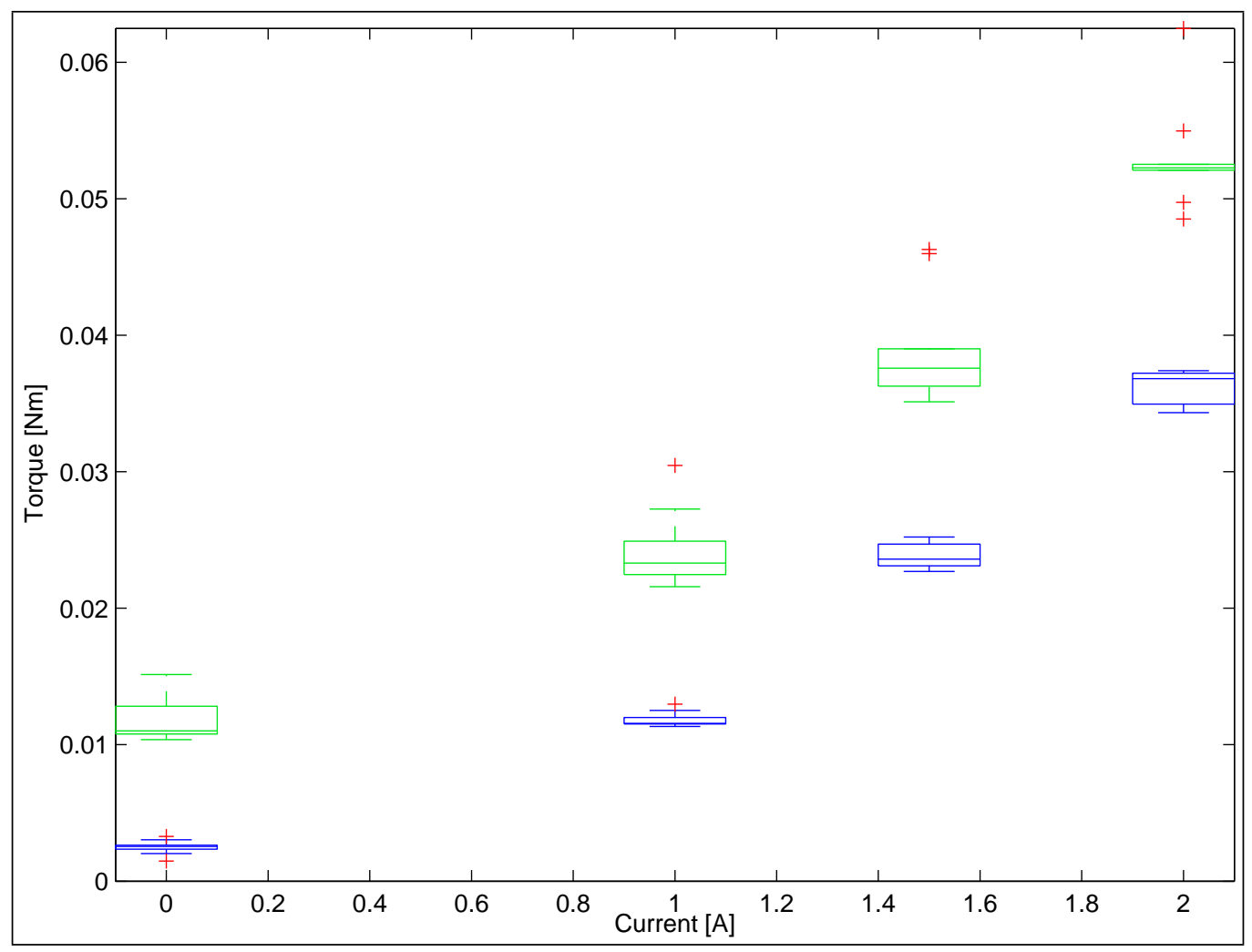

Figure 4.21: Calculated torque measurements of the paddle rotating at motor speeds of $10^{\circ} / \mathrm{s}$ (blue) and $75^{\circ} / \mathrm{s}$ (green), inside damper filled with MR fluid. The current was varied for each speed and the plot contains the 10 trials for each of the seven motor speeds. 


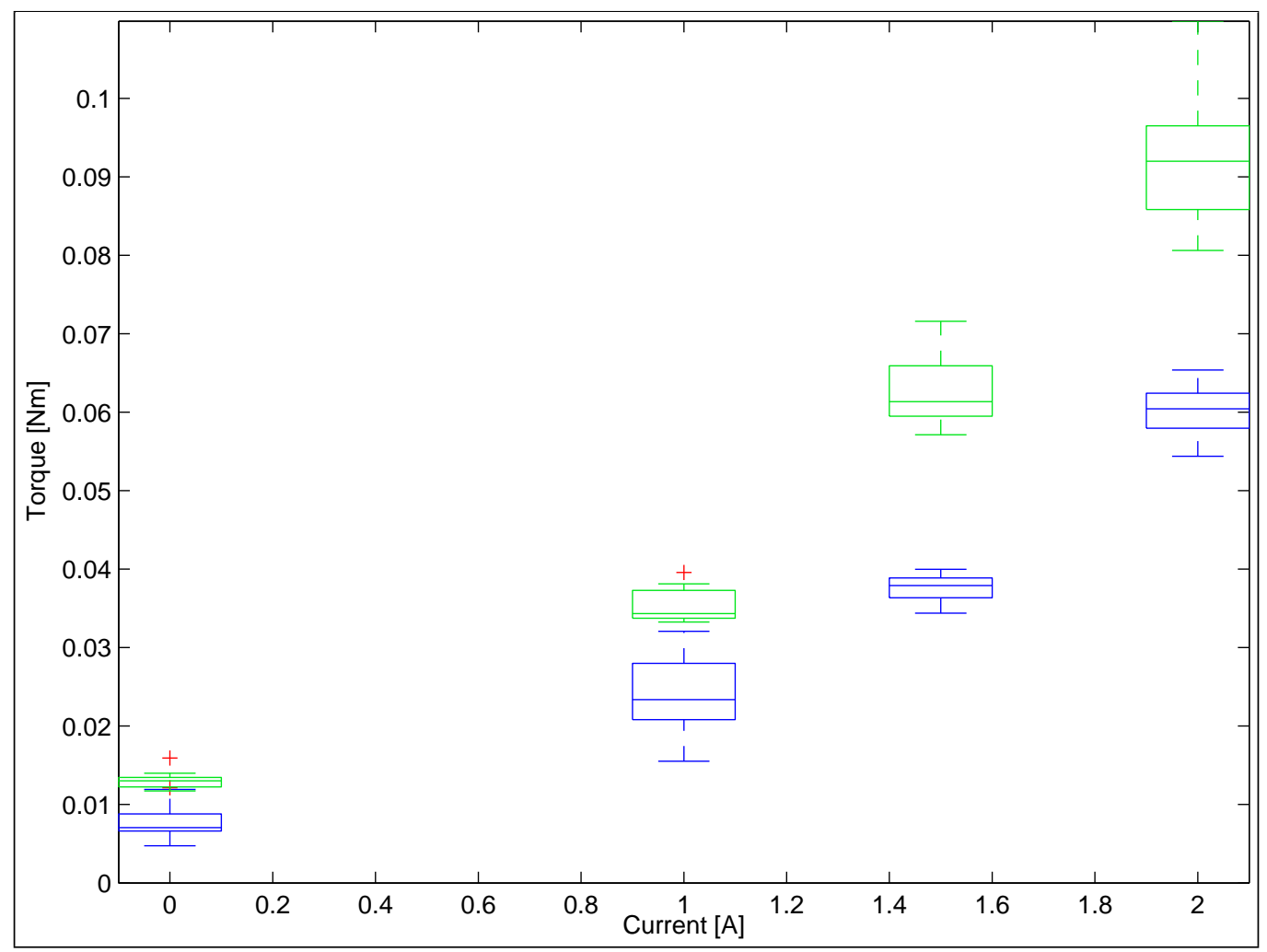

Figure 4.22: Calculated torque measurements of the helix rotating at motor speeds of $10^{\circ} / \mathrm{s}$ (blue) and $75^{\circ} / \mathrm{s}$ (green), inside damper filled with MR fluid. The current was varied for each speed and the plot contains the 10 trials for each of the seven motor speeds.

As observed in each of the plots, there are noticeable changes in the viscous torque with changes in the input current, indicating the ability to control the dampers. All three vane designs exhibit a non-linear relationship between the torque and input current.

The discs design contains the lowest mean off-state torque $(0 \mathrm{~A}$ and $10^{\circ} / \mathrm{s}$ ) of $0.0013 \mathrm{Nm}$ (standard error of $0.00018 \mathrm{Nm}$ ), and its largest mean torque ( $2 \mathrm{~A}$ and $75^{\circ} / \mathrm{s}$ ) was $0.024 \mathrm{Nm}$ (standard error of $0.00026 \mathrm{Nm}$ ). The largest possible viscous torque range of the discs within the range of motor speed and current settings was $0.022 \mathrm{Nm}$, corresponding to a change 
in force of approximately $4 \mathrm{~N}$. This small torque range could limit the flexibility of the damper, by not being able to provide for a range in strength capabilities of the users.

The paddle design contained the second lowest mean off-state torque of $0.0025 \mathrm{Nm}$ (standard error of $0.00016 \mathrm{Nm}$ ) and its highest calculate mean torque of $0.053 \mathrm{Nm}$ (standard error of $0.0012 \mathrm{Nm}$ ). The range of the viscous torque is $0.0505 \mathrm{Nm}$, over twice the torque range of the discs, which corresponds to an approximate force range of 9.2N.

The helix vane had the highest off-state torque value and the largest viscous torque range. The mean off-state torque was $0.0080 \mathrm{Nm}$ (standard error of $0.00076 \mathrm{Nm}$ ) and the highest mean torque was $0.0922 \mathrm{Nm}$ (standard error of $0.0026 \mathrm{Nm}$ ). This gave the helix a torque range of $0.084 \mathrm{Nm}$, which is an approximate force of $15 \mathrm{~N}$. However the helix vane was also the least consistent in operation as shown in Figure 4.22, with a standard deviation of $0.0083 \mathrm{Nm}$ at $2 \mathrm{~A}, 75^{\circ} / \mathrm{s}$, which is nearly a $20 \%$ variation of its total torque range. This large variation would make the helix design difficult to adequately control in a complete rehabilitation system.

\subsubsection{Selection of the vane design to further develop}

It was found that all three designs functioned adequately as dampers, although each interacted with the fluid differently.

The helix and discs vane designs were 3D printed due to the complexity of the required parts. The limited accuracy of 3D printing, coupled with the irregular inner diameter of the housing, often caused points of friction between the vane and the housing affecting the opposing force measurements. The complexities in those designs would cause the manufacturing cost for the helix and the assembly cost of the discs design to be high. In contrast the simple paddle design was able to be easily machined and would be low cost to manufacture.

The discs design contained the lowest off state torque, which is good 
for when the damper is passive, but it also had the lowest torque range, which limits the damper's functionality in the system and its flexibility in catering for a wide range of users. The helix design contained the highest viscous torque range but also the largest off-state torque. Also the inconsistency in the results of the helix design would increase the complexity of the control of the damper. The paddle design ranked second for both the off-state torque and torque range. It is interesting to note that there is nearly a $5 \mathrm{~N}$ difference between the opposing force ranges of each of the three designs.

Considering the complexity of the designs, cost to manufacture, and the performance of the damper, the paddle vane design was chosen to be further developed. The paddle was the most cost effective, simple design and was a good compromise between the off-state torque and the total range of the viscous torque of the damper.

\subsection{Further Experimentation with Paddle Design}

An experiment was conducted to determine the behaviour of the paddle in commercial MR fluid, using the brass housing, to validate the previous results from experiments conducted with the homemade MR fluid. The settling of both fluids were investigated to determine if the settling of iron particles would hinder the performance of the damper. The damper was scaled in size to reduce its mass and increase portability. The effect the size of the damper had on the generated force was investigated. Two alternative paddle designs were used to explore the hypothesis of the paddle's interaction with MR fluid and the effect of temperature on the viscous torque was measured. 


\subsubsection{Settling of homemade and commercial MR fluid}

An experiment was conducted to determine the effects of settling of the homemade and commercial MR fluid. MRHCCS4-B (80\% iron content) was purchased from Liquid Research Limited. Two containers were filled, one with homemade fluid and the other with commercial fluid. Both containers were vigorously shaken for five minutes and left to sit on a bench. After three days there was noticeable separation between the oil and iron in the homemade fluid. A couple of days later there was visible separation in the commercial fliud. However both fluids were able to be reconstituted when shaken for two minutes.

Two other factors mitigate the risk of fluid settling. Firstly as the paddle design causes the fluid to mix during operation settling was not considered to be an issue for the damper design. Secondly as the planned stroke rehabilitation device will be designed to be portable and strapped to a person's forearm, during operation the natural movements of the user will further reduce the settling of the iron particles.

\subsubsection{Paddle vane in commercial MR fluid}

The previous experiment containing homemade MR fluid in the damper was repeated using commercial fluid. This was to find any differences in the force measurements, and to determine whether the results previously obtained for the different vane designs were valid with the commercial fluid. The force-time response graph for $10^{\circ} / \mathrm{s}$ is shown in Figure 4.23 and the calculated viscous torque for changes in the input current and motor speed are shown in Figure 4.24. 


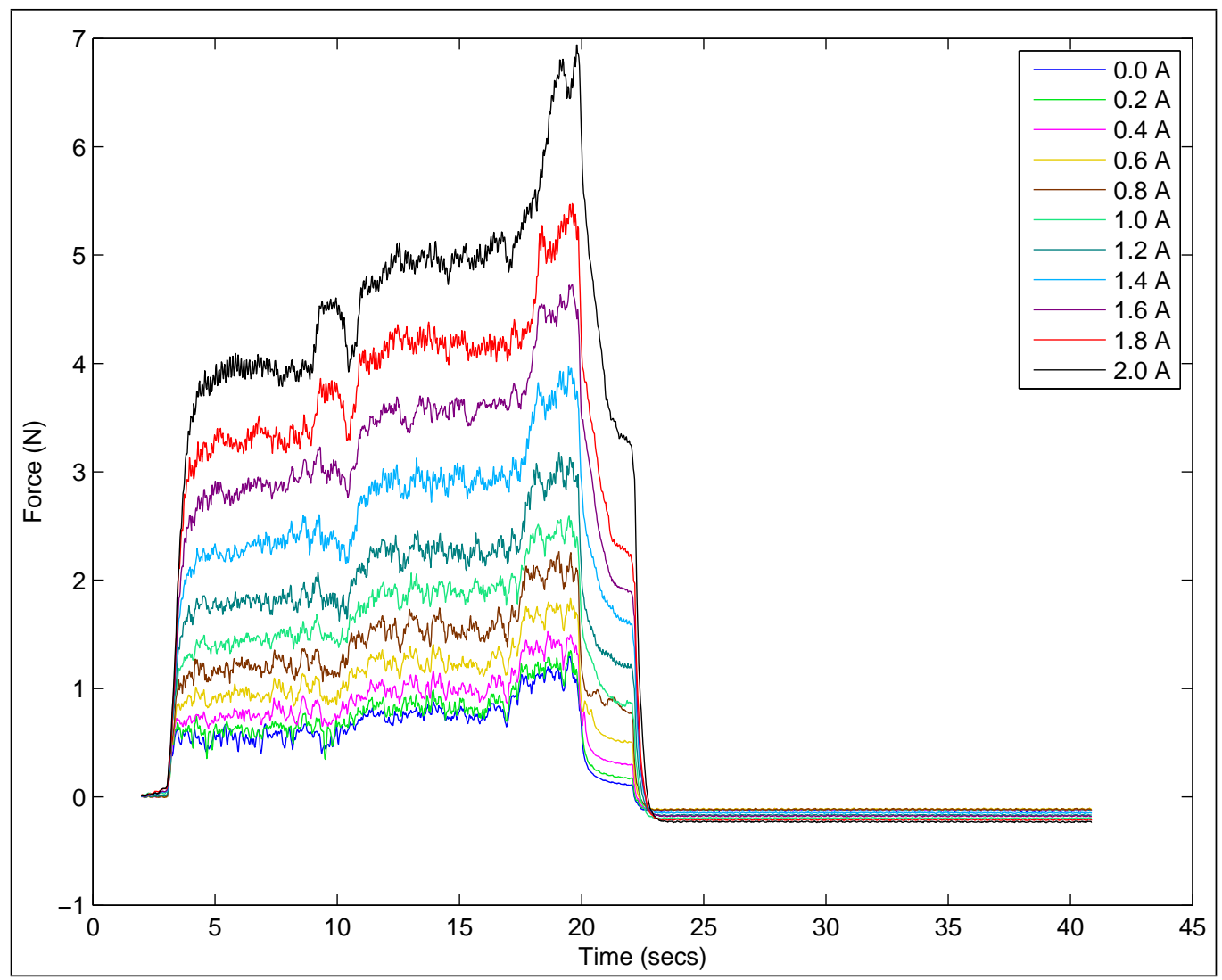

Figure 4.23: The paddle vane rotating inside the damper filled with commercial MR fluid with a change in the input current. The motor's speed was set to $10^{\circ} / \mathrm{s}$ and each graph is an average of 10 trials. Note the lower average force values and the smoother graphs when compared to the results for homemade fluid in Figure 4.16 


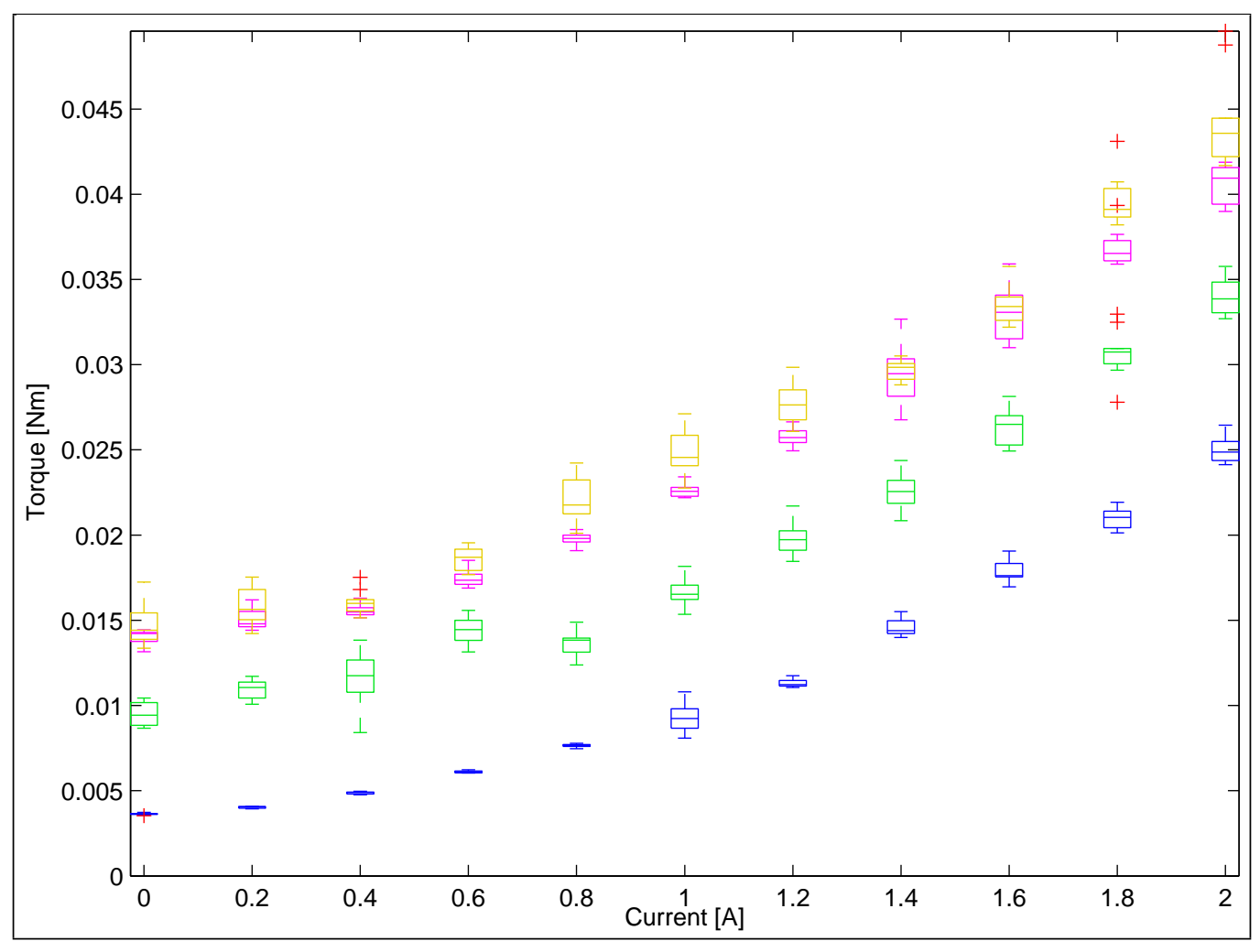

Figure 4.24: Calculated torque measurements for the paddle rotating inside the damper filled with commercial fluid, with varying motor speeds and changes in input current. The motor speeds are as follows: $10^{\circ} / \mathrm{s}$ (blue), 30\% $\mathrm{s}$ (green), 50\% (pink) and 75\% (yellow). Note the change in viscous torque between motor speeds (at the same input current) decreases as the speed increases.

Comparing the force results for the paddle in the commercial MR fluid (Figure 4.23) and the homemade fluid (Figure 4.16), the commercial fluid has smoother graphs but a lower averaged force. The change in the response may be due to the better quality ingredients and smaller iron particles used in the commercial fluid.

The mean off-state torque at $10^{\circ} / \mathrm{s}$ of the paddle in commercial fluid was $0.0036 \mathrm{Nm}$ (standard error of $0.000017 \mathrm{Nm}$ ) which was higher than 
the homemade fluid mean torque of $0.0025 \mathrm{Nm}(0.00016 \mathrm{Nm})$. The highest mean torque result of the commercial fluid at $75^{\circ} / \mathrm{s}(2 \mathrm{~A})$ was $0.044 \mathrm{Nm}$ (standard error of $0.00087 \mathrm{Nm}$ ), which was lower than the homemade fluid torque of $0.053 \mathrm{Nm}$ (standard error of $0.0012 \mathrm{Nm}$ ). The torque range of paddle in commercial fluid corresponds to an approximate force range of $7.3 \mathrm{~N}$, compared to $9.2 \mathrm{~N}$ in the homemade fluid. Though the torque is reduced it remains sufficient for practical use.

Both experiments, using the commercial and homemade fluid, show the same non-linear relationship between the viscous torque with changes to input current.

It is interesting to note that for the same current input the change in the viscous torque decreases, as the motor speed increases. This is different to the results seen in Figure 4.21, where the paddle was rotated in oil and with motor speeds of up to $200^{\circ} / \mathrm{s}$ there were still noticeable increases in the generated torque.

\subsubsection{Modifications to the housing}

Modifications to the design of the housing were required to reduce the elliptical movements of the shaft (seen in Section 4.3) and to make it easier to fill the damper and switch the vane designs when necessary. The new housing was also scaled down in size to reduce the mass of the damper and increase portability.

Bearings were used at the top and bottom of the housing to prevent the shaft from rotating around its vertical axis to reduce the horizontal elliptical movements. The use of bearings also minimised the rotational friction to reduce the off-state torque.

The screw caps that were used in the brass housing were replaced with press down caps, allowing both the damper to be filled and the vane designs to be switched more easily. An exploded view of the housing is shown in Figure 4.25 and its dimensions are shown in Figure 4.26. 
The electromagnet coil was again made from $0.71 \mathrm{~mm}$ diameter copper wire and had 355 turns, similar to that used with the brass housing.

The inside height of the housing was halved from the previous design reducing it from $80 \mathrm{~mm}$ to $40 \mathrm{~mm}$. The reduction in size of the damper also reduced the volume of MR fluid required, lowering the cost of the damper. To further reduce the weight of the previous brass housing, the new housing was made from PVC. PVC has a softening point of $55^{\circ} \mathrm{C}$, which is low but still above the specified system operating temperature of $34^{\circ} \mathrm{C}$.

The housing was machined to maintain a regular inner diameter. The mass of the housing including the MR fluid and electromagnet was $380 \mathrm{~g}$, which is less than the specified mass of $500 \mathrm{~g}$. The volume of fluid required in the larger damper was less than $70 \mathrm{ml}$, corresponding to less than 35 USD. The modified smaller size of the damper would reduce the cost of the fluid to below 17.5 USD. 


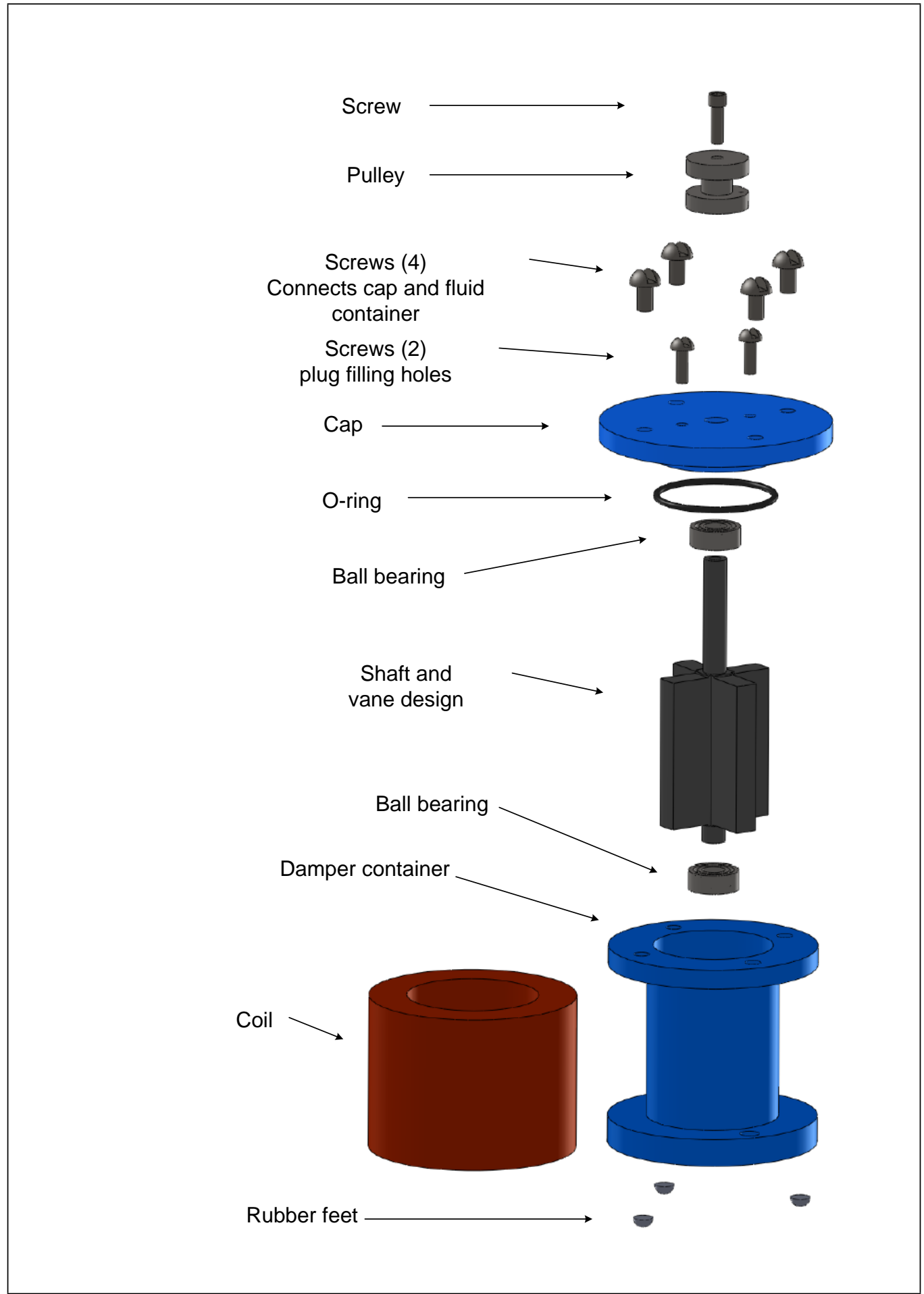

Figure 4.25: Exploded view of the new housing showing the parts required. 
In an attempt to investigate which of the two hypotheses proposed in Section 3.4.2 described the paddle's interaction with the fluid, a cross shaped vane was constructed (refer to Figure 4.26). If the first hypothesis, that the majority of the generated force is caused by the paddle slicing through the iron chains during operation is true, then doubling the number of the arms of the vane should effectively double the number of iron chains broken per unit time and should produce an increase in the opposing force. If the second hypothesis is true, such that the vane moves the fluid in bulk, then increasing the number of arms may slightly decrease the generated force, as some of the previous volume of fluid has now been replaced by the increased volume of the vane.

In either case reducing the height of the damper should produce a reduction in the generated force below that observed with the larger damper.

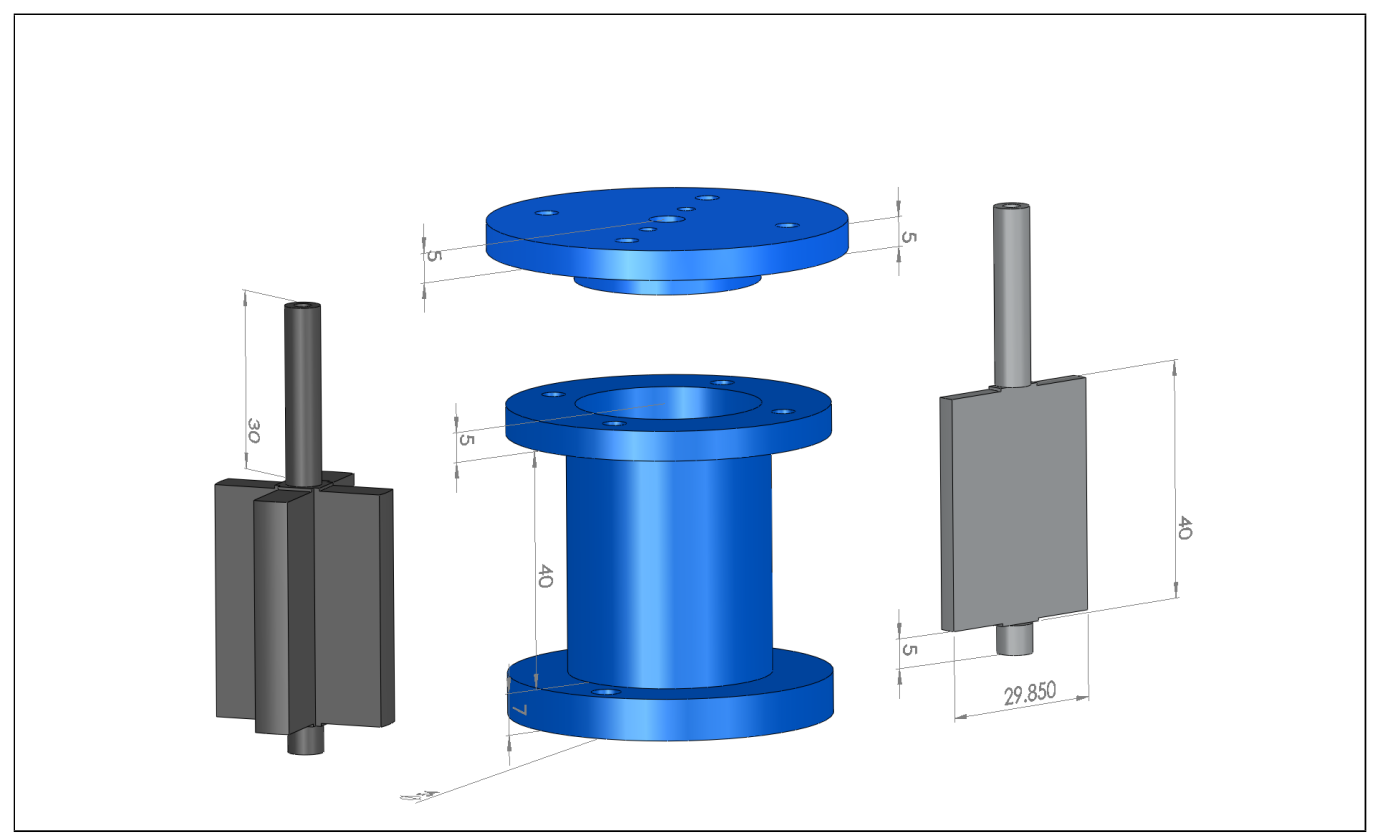

Figure 4.26: Render of the new housing and vane designs, with dimensions. Note the cross vane is on the left and the paddle vane on the right. The thickness of the arms of the vanes is $3.8 \mathrm{~mm}$. 


\subsubsection{Results of the two alternative vane designs in magneto- rheological fluid}

An experiment was conducted to determine what the effect of reducing the size of the housing had on the damper's performance. The experiment involved measuring the generated force produced by the cross and paddle vanes rotating in commercial MR fluid with changes to the input current and the motor's speed. The results were also used to compare the performance of the two vane designs, to help get a better understanding of how the vanes interact with the fluid.

The input current was varied to determine if scaling the size of the housing still allowed the interaction of the vanes in the fluid to function as a damper. A representative motor speed of $30^{\circ} / \mathrm{s}$ was chosen as this would be considered a moderate interaction speed for the user. The current was varied in steps of $0.2 \mathrm{~A}$ and the results for both the paddle and cross vane designs are shown in Figure 4.27. 


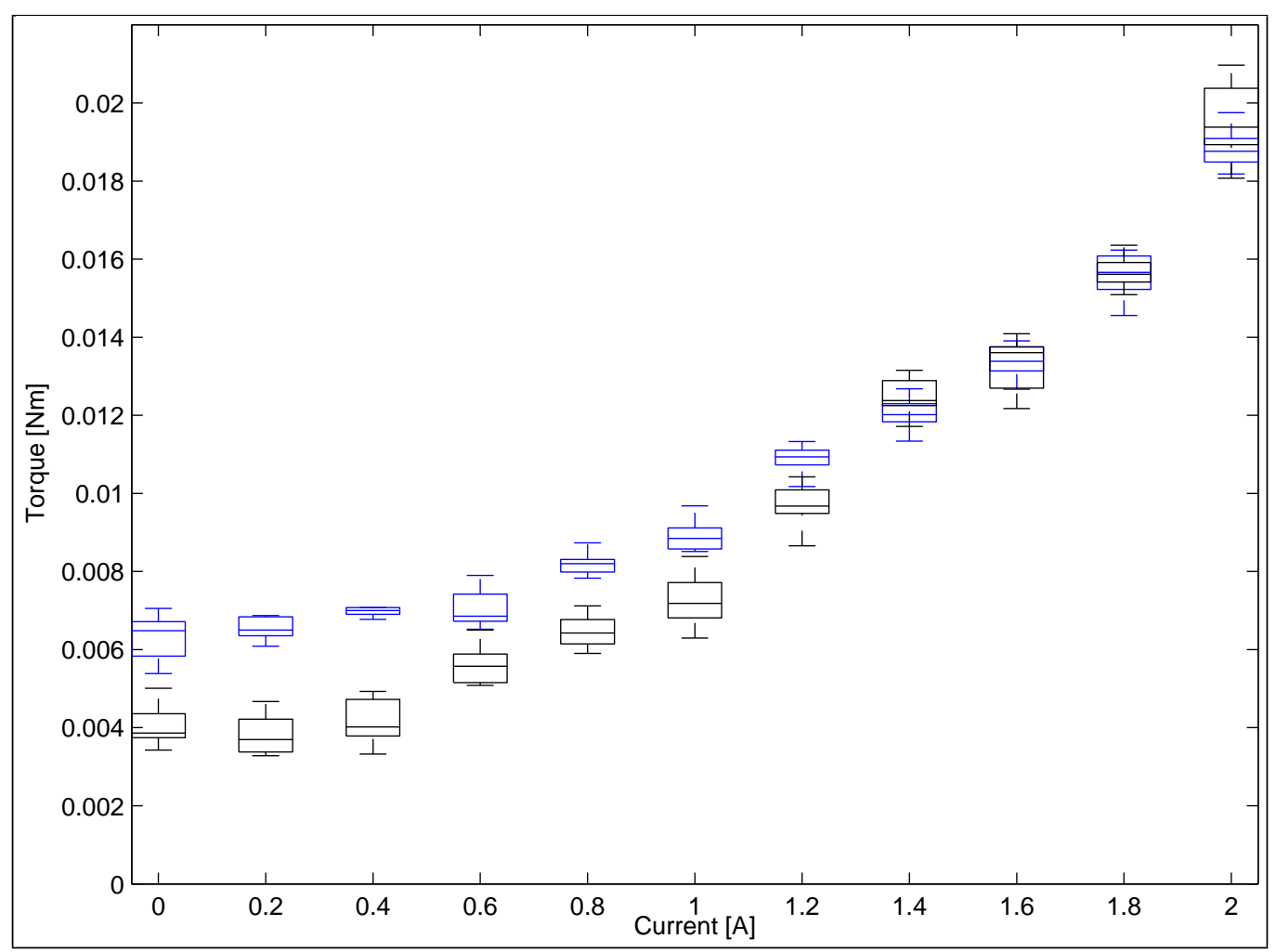

Figure 4.27: Calculated torque measurements of the alternative paddle (blue) and cross (black) vanes rotating in the MR filled damper at a motor speed of $30^{\circ} \mathrm{s}$. The current was varied from 0 to $2 \mathrm{~A}$ and the plot contains the 10 trials for each vane at each current setting.

The inclusion of the bearings at the top and bottom of the shaft prevented the shaft from 'wobbling' about its vertical axis and provided a smoother generated force response.

The results show the ability of both the vane designs to operate as a damper, as the viscous torque increases with increases in the current.

If the first hypothesis is true, then the cross design should have had a higher torque. This was found to not be the case. It is likely that the second hypothesis is true, that the majority of the generated force is caused by the vane moving the fluid in bulk, but further investigation is required to confirm this. 
Comparing the trend of the viscous torque with changes to input current for the paddle and cross designs, the cross contains a lower viscous torque at input currents below $1.4 \mathrm{~A}$, but then rises at an increased rate to match the magnitude of the torque results of the paddle. This result indicates that the cross vane would be better suited for the damper as it would have a lower off-state torque, but at higher currents it should be able to produce the equivalent torque ratings as the paddle.

The mean off-state torque at a motor speed of $30^{\circ} / \mathrm{s}$ for the cross was $0.0040 \mathrm{Nm}$ (standard error of $0.00016 \mathrm{Nm}$ ) and $0.0063 \mathrm{Nm}$ for the paddle (standard error of $0.00016 \mathrm{Nm}$ ). The mean torque at $2 \mathrm{~A}$ was $0.020 \mathrm{Nm}$ (standard error 0.0003) for the cross and $0.019 \mathrm{Nm}$ (standard error $0.00019 \mathrm{Nm}$ ) for the paddle. At the same motor speed, the paddle previously used in the brass housing with double the height had an mean off-state torque of $0.0095 \mathrm{Nm}$ (standard error $0.00022 \mathrm{Nm}$ ) and a mean torque of $0.034 \mathrm{Nm}$ (standard error $0.00033 \mathrm{Nm}$ ) with input current at $2 \mathrm{~A}$. As would be expected the results show that reducing the size of the damper reduces the off-state torque and also the range of the torque generated in the selected operating currents.

Another experiment was conducted using motor speeds varying from $5^{\circ} / \mathrm{s}$ to $75^{\circ} / \mathrm{s}$ and an input current of $1 \mathrm{~A}$. This was to further investigate the effects of changing the motor's speed while using the same input current. Although $5^{\circ} / \mathrm{s}$ was not in the expected range of operating speeds for the system (refer to Section 3.5.1), it was included in the experiment to demonstrate the trend of the results. 


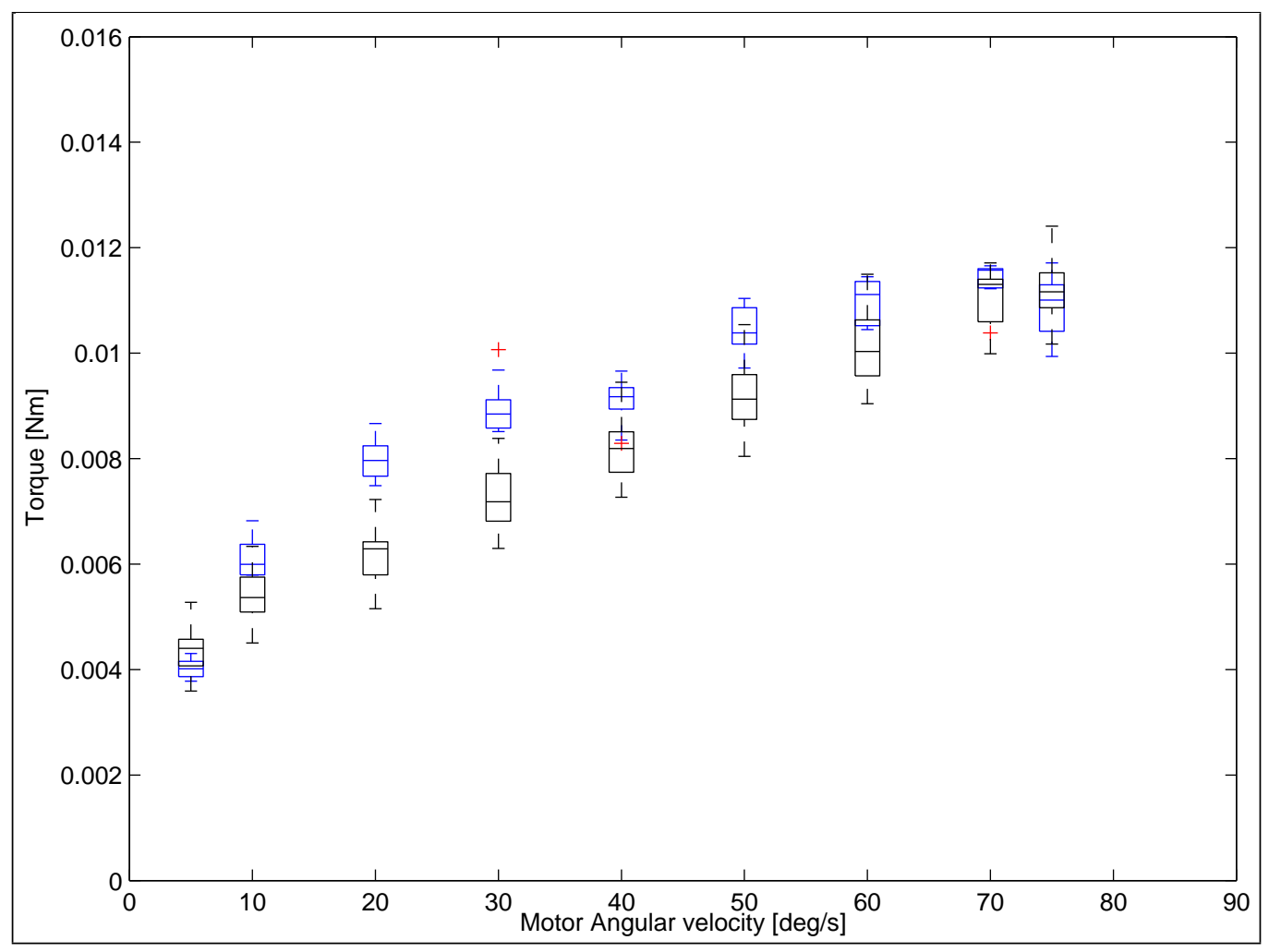

Figure 4.28: Calculated torque measurements of the alternative paddle (blue) and cross (black) vanes rotating in the MR filled damper with varying motor speeds and an input current of $1 \mathrm{~A}$. The plot contains the 10 trials for each vane at the different motor speed setting.

The results of the experiment show that eventually with an increase in the motor's speed there is no increase in the generated torque.

\subsubsection{Heating effects on the generated torque of the damper}

To demonstrate the effect of heating on the operation of the damper, the generated force of the paddle vane was tested over a period of 2 hours with a coil current continuously applied. A selected motor speed of $30^{\circ} / \mathrm{s}$ and an input current of $1 \mathrm{~A}$ was used for this test. The temperature from the LM35 sensor placed under the electromagnet was recorded. 


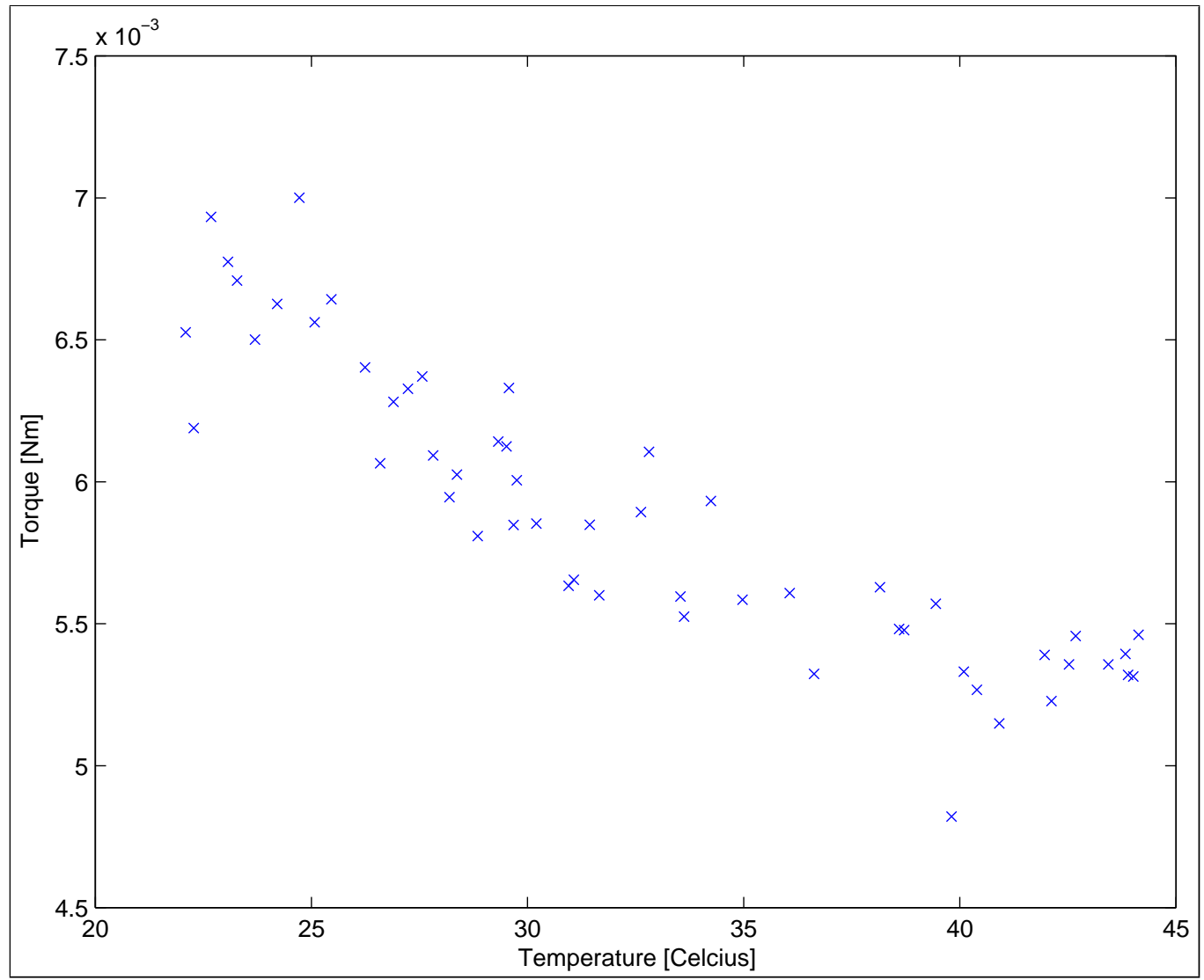

Figure 4.29: Alternative paddle vane rotating in the MR filled damper at a motor speed of $30^{\circ} \mathrm{s}$. A continuous coil current of $1 \mathrm{~A}$ is applied over a duration of approximately 2 hours. Each point represents the mean torque of each trial with the mean temperature of the electromagnet, during the operational period of the damper in a test cycle.

As shown in Figure 4.29, the fluid is noticeably affected by heating due to the viscosity decrease in the oil. Increasing the temperature decreases the viscous torque. The torque dropped by approximately $0.0015 \mathrm{Nm}$ from ambient temperature to the system specified limit of $34^{\circ} \mathrm{C}$, which is about a $16 \%$ variation from its mean torque of $0.009 \mathrm{Nm}$ (alternative paddle at $30^{\circ} / \mathrm{s}$ and $1 \mathrm{~A}$ with standard error of $0.00016 \mathrm{Nm}$ ). This corresponds to a small change of approximately $0.27 \mathrm{~N}$. 
However it should be noted that this test was conducted in worst case conditions, as it is unlikely that the rehabilitation device would be operated for 2 hours with a continuous coil current.

\subsection{Summary}

The three vane designs, discs, paddle and helix, were tested in oil with varying motor speeds. It was found that all three designs were able to generate an opposing force when rotated, which was a good indication that the vanes were feasible for use in a damper. The helix design produced the greatest generated force of approximately $10 \mathrm{~N}$ (at 200\% s). However the consistency of its torque was the least of the three designs, varying by up to $30 \%$ of its total.

The three vane designs were tested in MR fluid to determine the controllability and consistency of their performance. It was found that all three designs functioned as dampers, due to the range of torque produced. It was noted that each interacted with the fluid differently, which could be useful when matching a force profile to a patient's need. There were noticeable distinctions in the viscous torque with changes in the input current, indicating the ability to dynamical control the dampers. All three vane designs exhibited a predictable non-linear relationship between the torque and input current.

At the lowest motor speed setting of $10^{\circ} / \mathrm{s}$, the discs design contained the lowest off-state mean torque of $0.0013 \mathrm{Nm}$ (standard error of $0.00018 \mathrm{Nm}$ ), followed by the paddle at $0.0025 \mathrm{Nm}$ (standard error of $0.00016 \mathrm{Nm}$ ) and then the helix $0.0080 \mathrm{Nm}$ (standard error of $0.00076 \mathrm{Nm}$ ). At a motor speed of $75^{\circ} / \mathrm{s}$ and an input current of $2 \mathrm{~A}$, the helix produced the highest generated torque $0.092 \mathrm{Nm}$ (standard error of $0.0026 \mathrm{Nm}$ ), then the paddle $0.053 \mathrm{Nm}$ (standard error of $0.0012 \mathrm{Nm}$ ) and lastly the discs $0.024 \mathrm{Nm}$ (standard error of $0.00026 \mathrm{Nm}$ ). Once again the consistency of the helix results were a concern with a standard deviation of $0.0083 \mathrm{Nm}$, at 
$2 \mathrm{~A}$ and $75^{\circ} / \mathrm{s}$, which was nearly a $20 \%$ variation of its total torque range.

Considering the complexity of the designs, cost to manufacture, and the performance of each vane design, the paddle was chosen to be further developed. In comparison to the three designs, the paddle was a good compromise between the off-state torque and the total range of the viscous torque of the damper.

Using the paddle design the experiment was repeated with commercial MR fluid to determine if there were noticeable differences between the homemade and commercial fluid. The commercial fluid provided smoother graphs but lowered the generated torque (at $75^{\circ} / \mathrm{s}$ and $2 \mathrm{~A}$ ) from $0.053 \mathrm{Nm}$ (standard error of $0.0012 \mathrm{Nm}$ ) to $0.044 \mathrm{Nm}$ (standard error of $0.00087 \mathrm{Nm}$ ). The experiments using the commercial and homemade fluid both agreed in the trend of the results, showing that the viscous torque increased with an increase in input current. This again demonstrated the controllability of the damper. Both results also showed the same nonlinear, monotonic relationship between the viscous torque and input current.

The housing was halved in height to reduce the size and mass of the damper and to determine the effect the size of the damper had on the generated force. As expected the results indicated that reducing the size of the damper caused a reduction in the viscous torque. Bearings were used to address the periodic oscillations in the force graphs believed to have been caused by the movement of the shaft about its vertical axis, and friction between the vane and housing. The periodic oscillations that were previously seen in the force response were removed with the addition of bearings. This supported the hypothesis that the spikes in the force graphs were due to mechanical issues rather than a property of the MR fluid.

A temperature based experiment found that the MR fluid was negatively affected by heating, as increasing the temperature caused a decrease in the torque. The $16 \%$ change in the torque is significant enough that a controller would need to be considered when implementing the damper 
in a stroke rehabilitation device.

Overall a simple damper was developed using a paddle vane design, such that its viscous torque could be dynamically controlled with input current, to produce repeatable measurements in its operating temperature range of below $34^{\circ} \mathrm{C}$. 


\section{Chapter 5}

\section{Conclusion}

The aim of the research was to determine whether the use of magnetorheological fluid in a controllable damper is viable for use in a stroke rehabilitation device. This was to enable the project partners to decide whether to continue the development of a magneto-rheological damper in a rehabilitation device for people affected by stroke.

\subsection{Conclusion}

It was found that the viscous torque of the developed rotary damper containing magneto-rheological fluid can be dynamically controlled with changes in input current in the range between 0-2 A. The non-linear but monotonic relationship between the viscous torque and the current indicate that it is feasible to control the damper, but a non-trivial controller will be required. The damper was able to generate repeatable viscous torque whilst operating below $34^{\circ} \mathrm{C}$, the specified operating temperature of the system. It was found that decreasing the height of the damper reduced the range of the viscous torque. 


\subsection{Contribution}

The damper was designed to be used in a stroke rehabilitation device that is able to exercise and strengthen a person's hand. The damper needed to be dynamically controllable to vary the level of difficulty of the exercise during operation.

A test rig was designed and constructed to sample and record the force and temperature measurements of the damper. It was able to accommodate for dampers ranging in height from 55 to $140 \mathrm{~mm}$.

A rotary damper configuration was chosen due to its ability to be made compact and avoid fluid leakage. The majority of rotary dampers currently available use a disc design and require tight tolerances, which increases the complexity and cost of the damper. This study explored alternative designs to allow the damper to operate with looser tolerances and to be more cost effective.

The disc design was used and the alternative designs were the paddle and helix. All three designs were able to operate as dampers. The paddle design was chosen as it was found to be a good match for the requirements of the stroke rehabilitation device because of its performance and ease of manufacture. However if the need arose it is possible to use the other two designs to change the performance profile of the damper, such as changing the vane design used depending on the user's progress.

Previous problems with using magneto-rheological fluid include the leakage of the fluid, high off-state force or torque, settling of iron particles and heating of the fluid. As a rotary damper was used the leaking of the fluid was not an issue and the scale of the damper allowed the offstate torque to be small. The choice of the paddle design allowed the iron particles to disperse easily during operation, therefore settling was not considered a problem. It was found that with a continuous input current, the performance of the damper was affected by changes in temperature. During the operation of the damper in a stroke rehabilitation system, the 
temperature will need to be monitored and the control adjusted accordingly.

Magneto-rheological fluid is expensive per volume of fluid, but this work has showed that a practicable torque response could be achieved using a low volume of fluid. The largest damper developed in this study used less than $70 \mathrm{ml}$ of fluid which corresponds to less than 35 USD.

Historically stroke rehabilitation devices have focussed on repetitive exercises and often neglected strength training. The addition of a controllable damper allows a stroke rehabilitation device to become more versatile. The ability of the damper to be dynamically controlled allows the opposing force of the device to be changed, varying the level of difficulty of the exercise. This allows the device to adapt as the user improves motor control, continually challenging the user and increasing muscle strength. The damper will also be used to provide compliance to the system. This allows unintended forces caused by the user from spasms, or mechanical noise or oscillations caused by the device to be dampened, to protect both the user and device.

\subsection{Future Work}

The next stage of the study is to develop a prototype using cost effective materials and to begin clinical trials. The trials will be used to determine if the magnitude of the viscous torque, currently generated by the paddle vane damper, is adequate for the operation of the device. It may be found that multiple dampers could be required to provide flexibility in the device. This could include having a damper associated with each finger, to individually vary the resistance to finger movements. Any other modifications will be made to the damper before designing for manufacture, to allow the damper to be used in a stroke rehabilitation device. 


\subsection{Final Statement}

The use of magneto-rheological fluid was found to be viable solution for use in a low cost stroke rehabilitation device. 


\section{References}

[1] "Stroke, cerebrovascular accident." http://www. emro. who. int/ health-topics/stroke-cerebrovascular-accident/. Accessed: 12/09/2012.

[2] C. Btefisch, H. Hummelsheim, P. Denzler, and K.-H. Mauritz, "Repetitive training of isolated movements improves the outcome of motor rehabilitation of the centrally paretic hand," Journal of the Neurological Sciences, vol. 130, no. 1, pp. 59-68, 1995.

[3] A. Pascual-Leone, A. Amedi, F. Fregni, and L. B. Merabet, "The plastic human brain cortex," Annual Review of Neuroscience, vol. 28, no. 1, pp. 377-401, 2005.

[4] J. W. Krakauer, "Motor learning: its relevance to stroke recovery and neurorehabilitation," Current Opinion in Neurology, vol. 19, no. 1, pp. 84-90, 2006.

[5] S. Mann, "Physiotherapist, Kenepuru Hospital NZ." Personal Communication: August 2010.

[6] K. Jordan and M. King, "Augmented reality assisted upper limb rehabilitation following stroke," Augmented Reality Some Emerging Application Areas, 2011.

[7] L. Austin, "Stroke survivor." Personal Communication: October 2011. 
[8] R. Loureiro, B. Lamperd, C. Collin, and W. Harwin, "Reach grasp therapy: Effects of the gentle/g system assessing sub-acute stroke whole-arm rehabilitation," in Rehabilitation Robotics, 2009. ICORR 2009. IEEE International Conference on, pp. 755 -760, june 2009.

[9] N. Ifejika-Jones and A. Barrett, "Rehabilitationemerging technologies, innovative therapies, and future objectives," Neurotherapeutics, vol. 8, pp. 452-462, 2011.

[10] "Im-able - able-x." http://www.im-able.com/en. Accessed: 22/09/2012.

[11] "Meditouch - hand tutor." http://www.meditouch.co.il/ index. aspx?id=2433. Accessed: 22/09/2012.

[12] "Tyromotion - amadeo." http://www.tyromotion.com/en/ products / amadeo/overview.html. Accessed: 22/09/2012.

[13] C. Takahashi, L. Der-Yeghiaian, V. Le, and S. Cramer, "A robotic device for hand motor therapy after stroke," in Rehabilitation Robotics, 2005. ICORR 2005. 9th International Conference on, pp. 17 - 20, june-1 july 2005.

[14] R. Loureiro, B. Lamperd, C. Collin, and W. Harwin, "Reach grasp therapy: Effects of the gentle/g system assessing sub-acute stroke whole-arm rehabilitation," in Rehabilitation Robotics, 2009. ICORR 2009. IEEE International Conference on, pp. 755 -760, june 2009.

[15] R. Ham, T. Sugar, B. Vanderborght, K. Hollander, and D. Lefeber, "Compliant actuator designs," Robotics Automation Magazine, IEEE, vol. 16, pp. 81 -94, september 2009.

[16] A. Rajendran, C. Hollitt, and W. N. Browne, "Investigating the use of magneto-rheological fluid in an active compliant actuator for a stroke 
rehabilitation system," in Robotics and Automation, 2011. Proceedings. 13th Australasian Conference on, 2011.

[17] A. Rajendran, "Hand rehabilitation using compliant actuation." Final year project report. Victoria University of Wellington, 2010.

[18] R. Budynas and K. Nisbett, Shigleys Mechanical Engineering Design, Eight Edition. McGraw-Hill Companies, 2006.

[19] E. Ungar and K. Stroud, "A new approach to defining human touch temperature standards," in Environmental Systems, 2010. ICES 2010. Proceedings. 40th International Conference, July 2009.

[20] "LORD Corporation." http://www.lord.com/ products-and-solutions/magneto-rheological- $\ 28 \mathrm{mr} \backslash$ ○29/mr-store.xml. Accessed: 14/09/2012.

[21] "Liquids Research Limited." http://liquidsresearch.co. uk/en-GB/ordering_information-60.aspx.

Accessed: $14 / 09 / 2012$.

[22] "Hward." http://www. strokerobot.com/default.aspx. Accessed: 22/09/2012.

[23] R. Jacob, “High-speed magnetic fluid clutch." Patent. 2622713, December 1952.

[24] M. R. Jolly, J. W. Bender, and J. D. Carlson, "Properties and applications of commercial magnetorheological fluids," Journal of Intelligent Material Systems and Structures, vol. 10, no. 1, pp. 5-13, 1999.

[25] D. J. Klingenberg, "Magnetorheology: Applications and challenges," AIChE Journal, vol. 47, no. 2, pp. 246-249, 2001.

[26] R. Turczyn and M. Kciuk, "Preparation and study of model magnetorheological fluids.," Journal of Achievements in Materials and Manufacturing Engineering, vol. 27, no. 2, pp. 131-134, 2008. 
[27] M. T. Avraam, MR-fluid brake design and its application to a portable muscular rehabilitation device. PhD thesis, Universite Libre de Bruxelles, 2009.

[28] D. A. Rosenbaum, Human Motor Control. Academic Press Limited, 1991.

[29] J. H. Park and O. O. Park, "Electrorheology and magnetorheology," Korea-Australia Rheology Journal, vol. 13, pp. 13-17, march 2001.

[30] F. D. Goncalves and J. D. Carlson, "An alternate operation mode for mr fluidsmagnetic gradient pinch," in Electrorheological Fluids and Magnetorheological Suspensions. 11th Conference, August 2009.

[31] G. Savioz, V. Ruchet, and Y. Perriard, "Study of a miniature magnetorheological fluid actuator for haptic devices," in Advanced Intelligent Mechatronics (AIM), 2010 IEEE/ASME International Conference on, pp. $1197-1202$, july 2010.

[32] B. Kasemi, A. Muthalif, M. Rashid, and M. Rahman, "Optimizing dynamic range of magnetorheological fluid dampers: Modeling and simulation," in Mechatronics (ICOM), 2011 4th International Conference On, pp. $1-4$, may 2011.

[33] S. Winter and M. Bouzit, "Use of magnetorheological fluid in a force feedback glove," Neural Systems and Rehabilitation Engineering, IEEE Transactions on, vol. 15, pp. 2-8, march 2007.

[34] H. Herr and A. Wilkenfeld, "User-adaptive control of a magnetorheological prosthetic knee," Industrial Robot: An International Journal, vol. 30, no. 1, pp. 42-55, 2003.

[35] M. Avraam, M. Horodinca, P. Letier, and A. Preumont, "Portable smart wrist rehabilitation device driven by rotational mr-fluid brake 
actuator for telemedecine applications," in Intelligent Robots and Systems, 2008. IROS 2008. IEEE/RSJ International Conference on, pp. 1441 -1446, sept. 2008.

[36] B. Liu, W. H. Li, P. B. Kosasih, and X. Z. Zhang, "Development of an mr-brake-based haptic device," Smart Materials and Structures, vol. 15, no. 6, p. 1960, 2006.

[37] S. Allin, Y. Matsuoka, and R. Klatzky, "Measuring just noticeable differences for haptic force feedback: implications for rehabilitation," in Haptic Interfaces for Virtual Environment and Teleoperator Systems, 2002. HAPTICS 2002. Proceedings. 10th Symposium on, pp. 299 -302, 2002.

[38] X. Pang, H. Z. Tan, and N. Durlach, "Manual discrimination of force using active finger motion," Perception and Psychophysics, vol. 49, pp. 531-540, march 1991.

[39] S. S. Bhavikatti and K. G. Rajashekarappa, Engineering Mechanics. New Age International Ltd, 1998.

[40] "Magnetorheological fluid." http://haptics.bluwiki.com/ go/Magnetorheological_Fluid. Accessed: 03/10/2012. 
Appendix A

\section{LabVIEW Block Diagram}




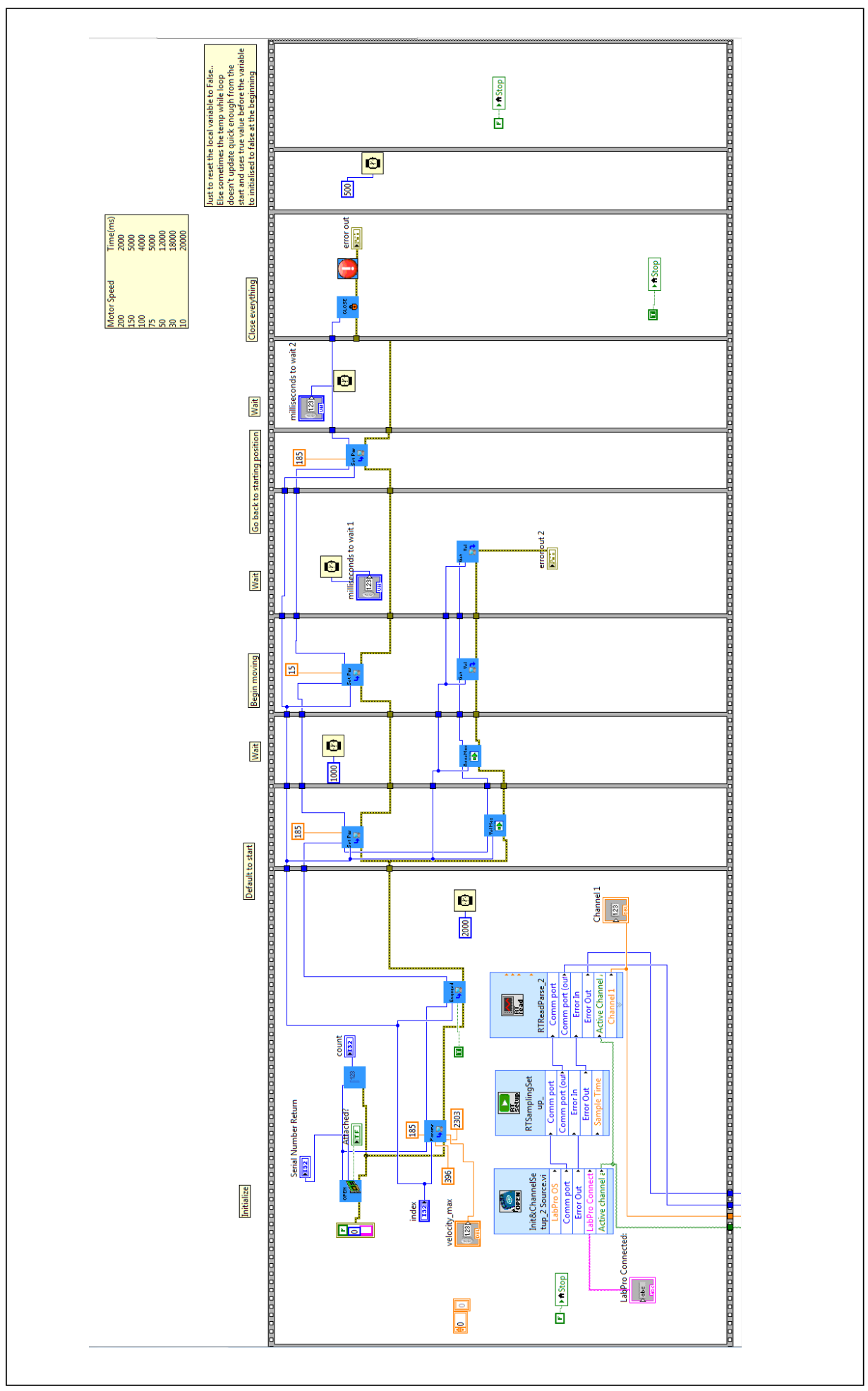

Figure A.1: Block Diagram of LabVIEW program Part A 


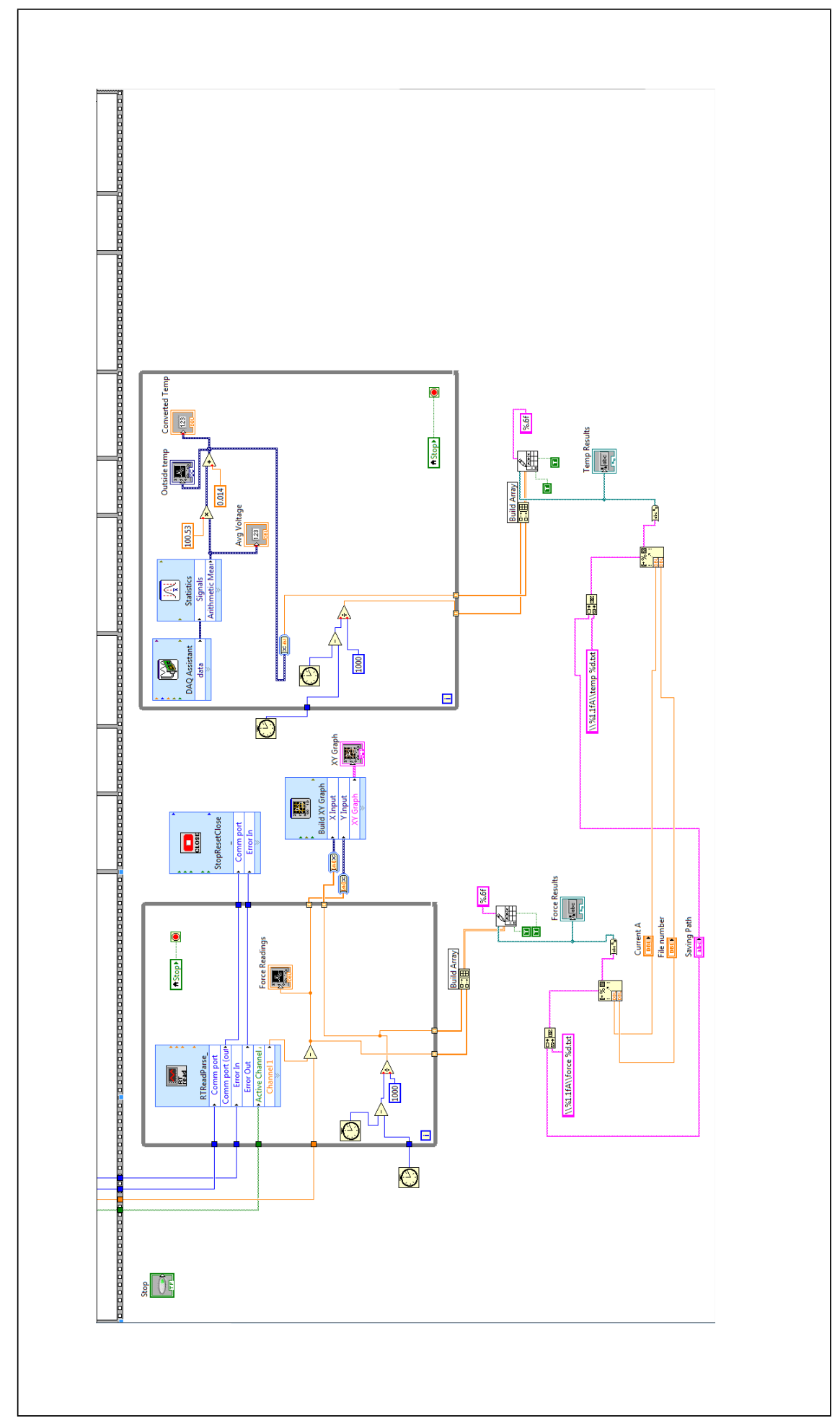

Figure A.2: Block Diagram of LabVIEW program Part B 


\section{Appendix B}

\section{Water Filled Damper Results}

\section{B.1 Water: Discs Design}

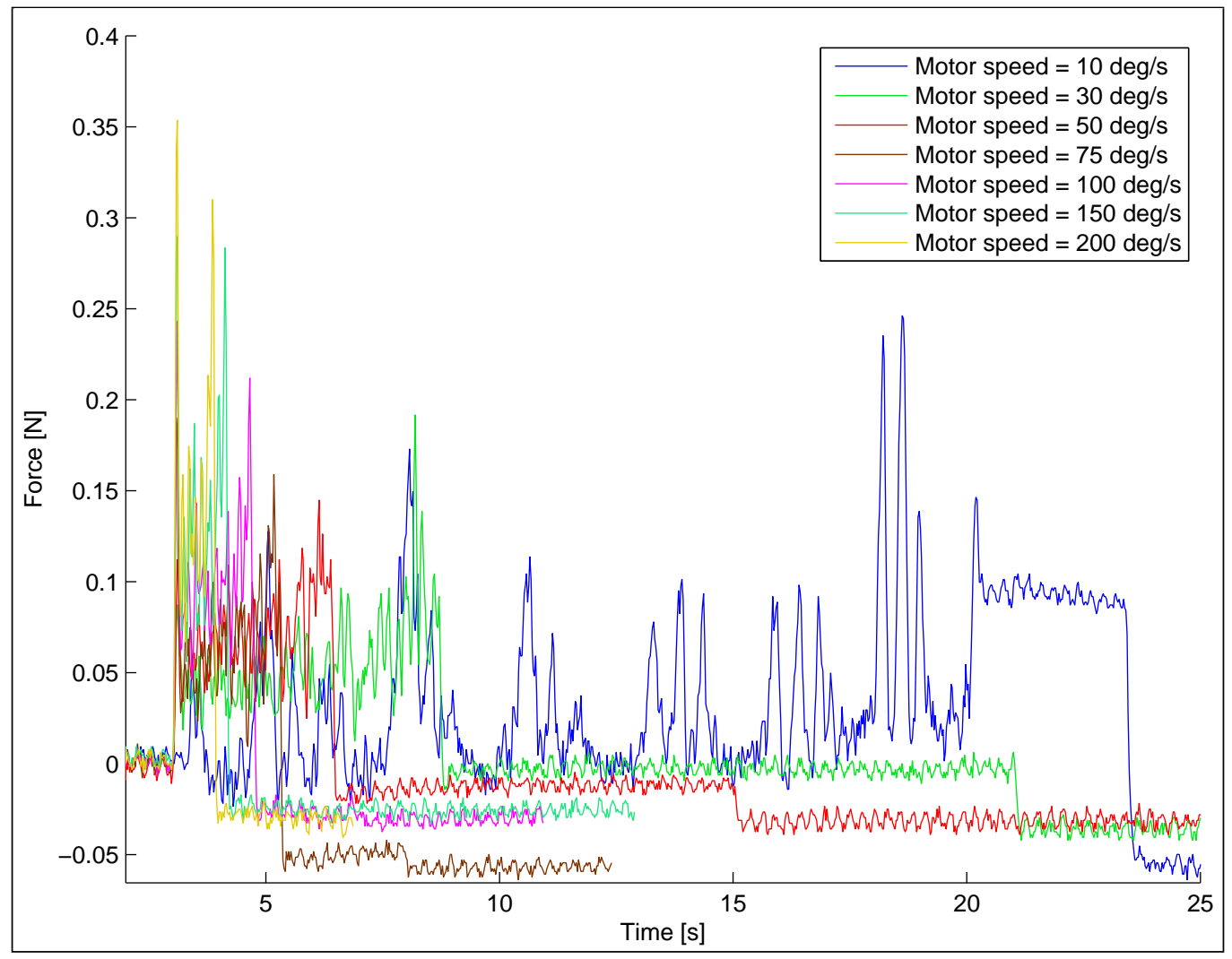

Figure B.1: The discs vane rotating inside the water filled damper with varying motor speeds where each graph represents an average of 10 trials. Note the drop in opposing force when compared to the results of the damper containing only air in Figure 4.1 


\section{B.2 Water: Flat Paddle Design}

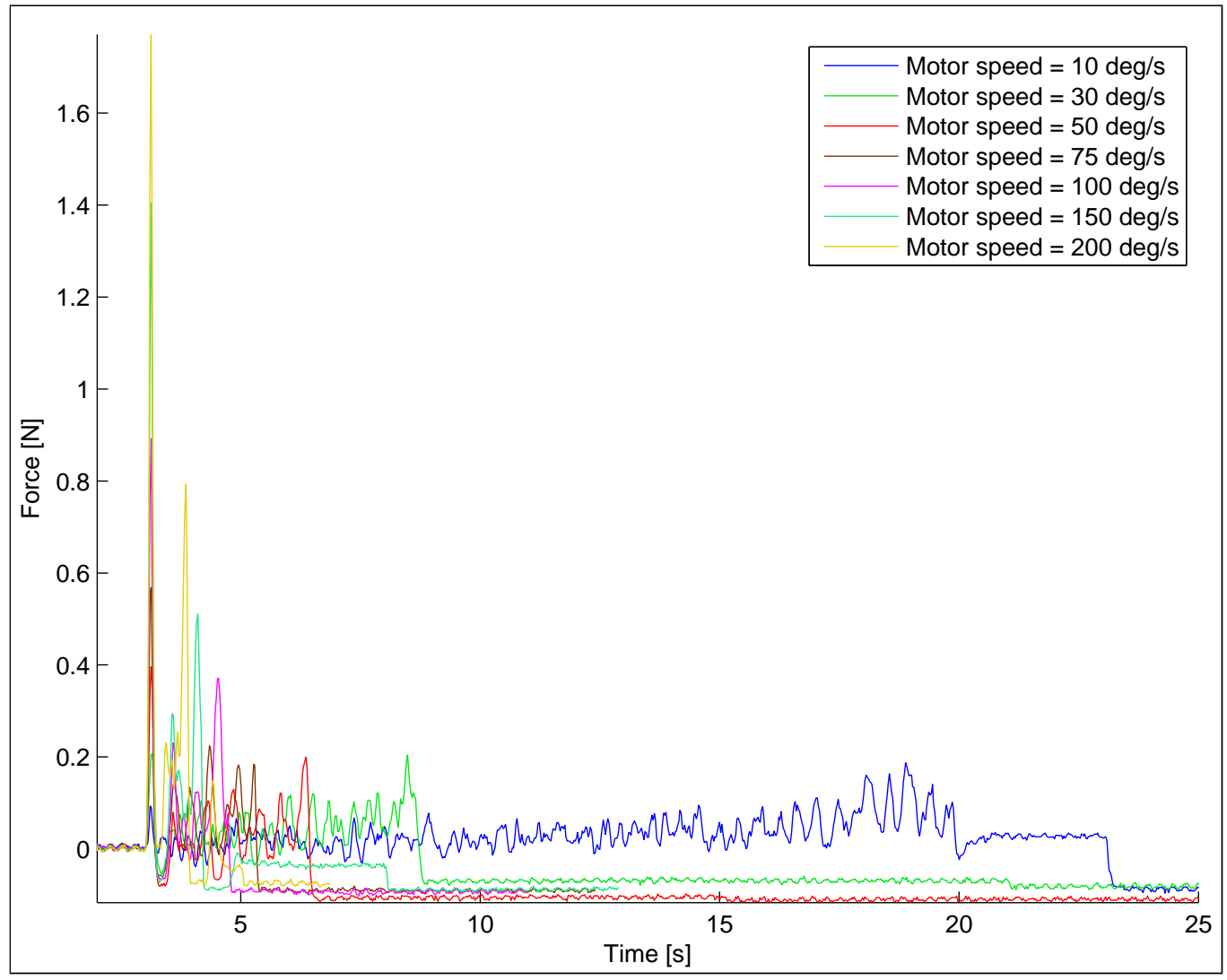

Figure B.2: The paddle vane rotating inside the water filled damper with varying motor speeds where each graph represents an average of 10 trials. 


\section{B.3 Water: Helix Design}

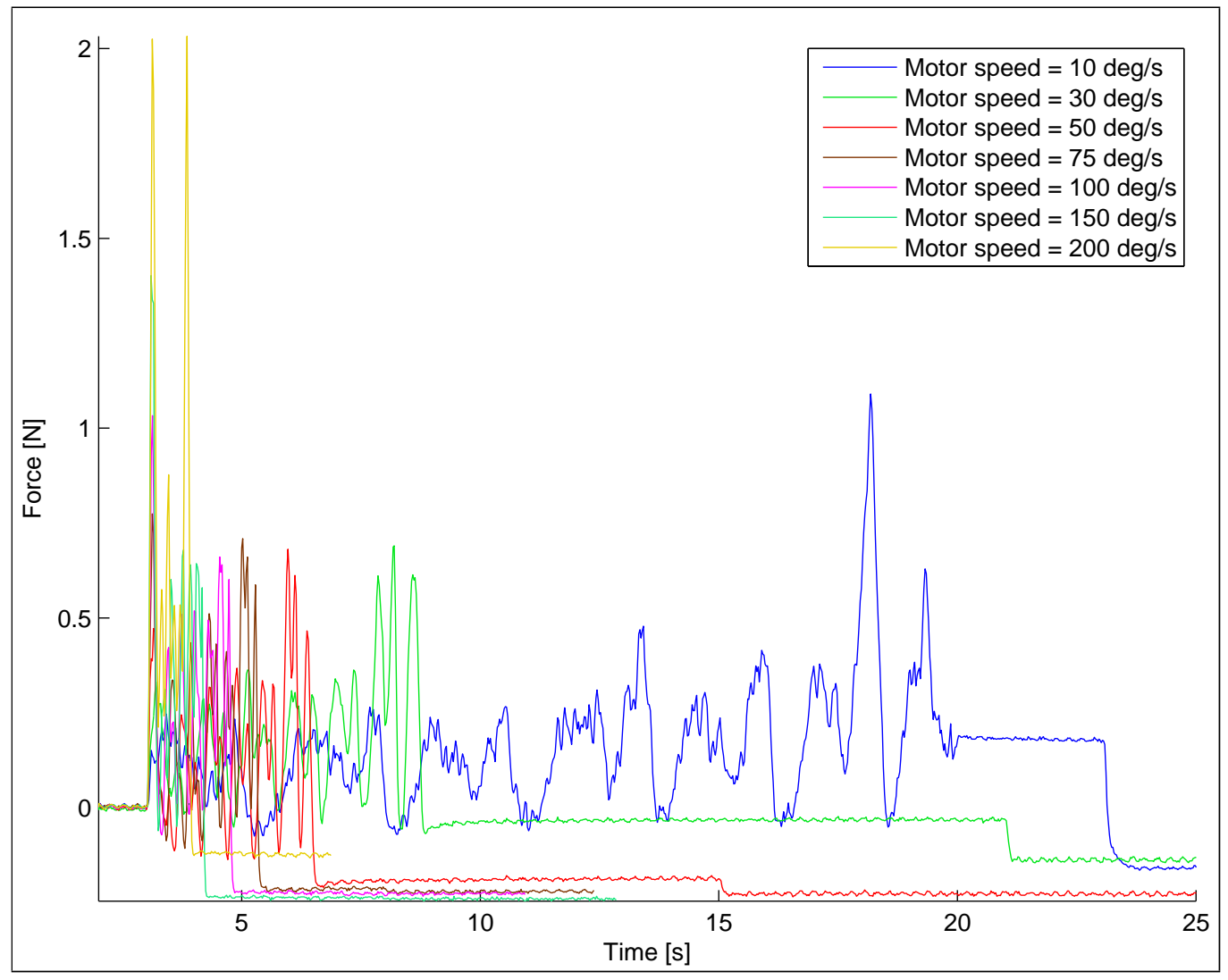

Figure B.3: The helix vane rotating inside the water filled damper with varying motor speeds where each graph represents an average of 10 trials. 